


\title{
Contemporary Topics in Graduate Medical Education
}

\author{
Edited by Stanislaw P. Stawicki, \\ Michael S. Firstenberg, James P. Orlando \\ and Thomas J. Papadimos
}



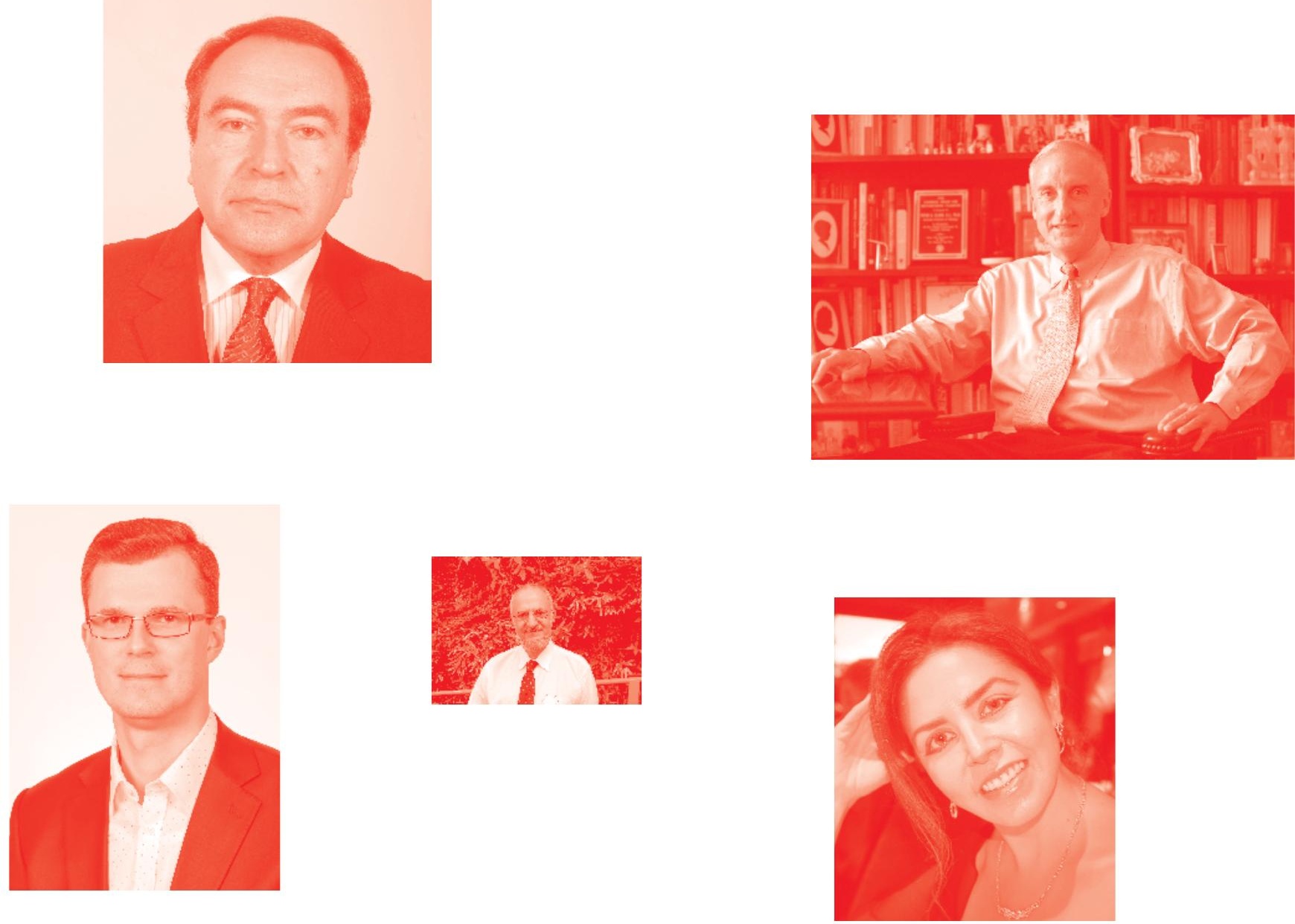

Supporting open minds since 2005
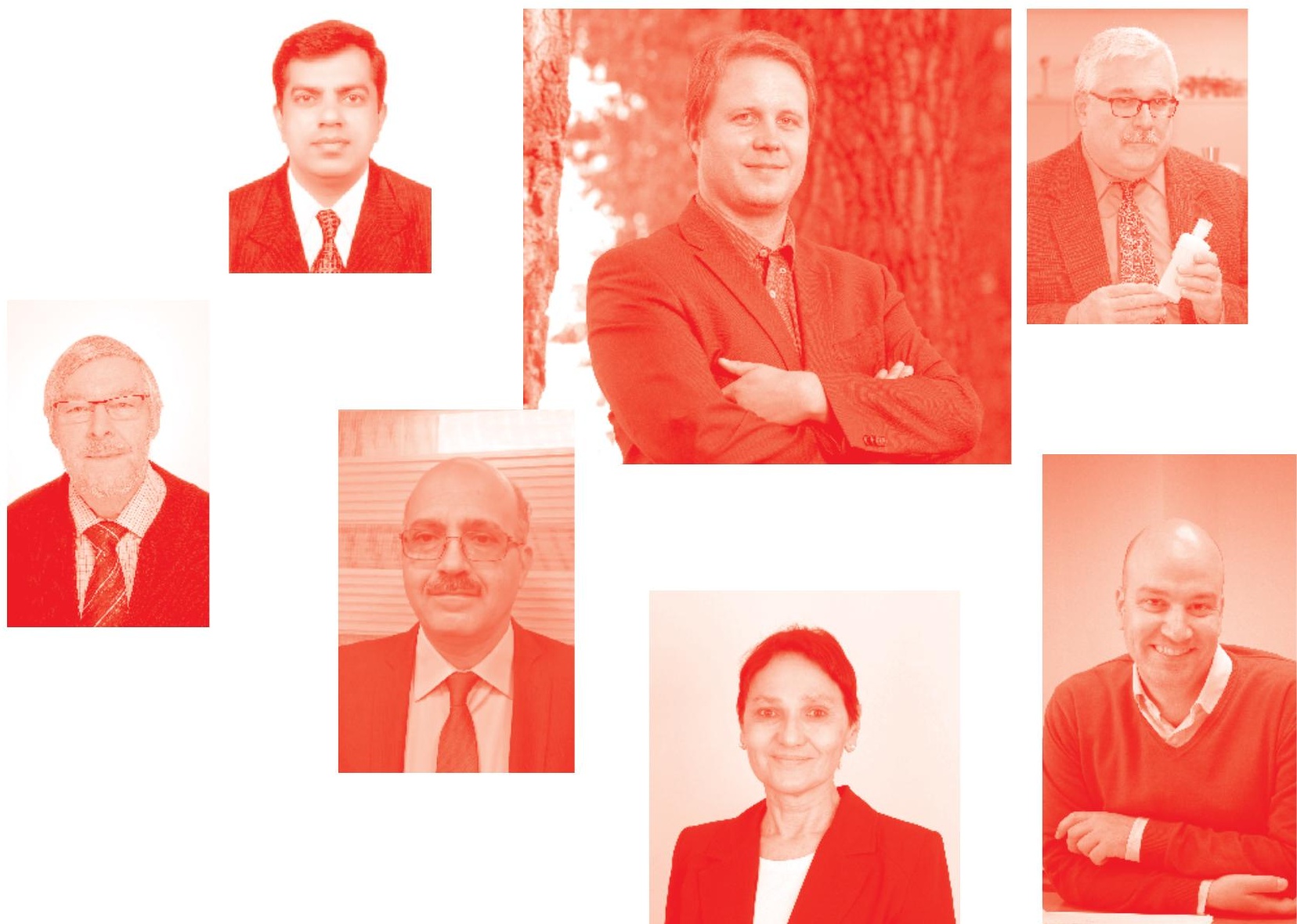
Contemporary Topics in Graduate Medical Education

http: //dx. doi . org/10.5772/intechopen . 79594

Edited by Stanislaw P. Stawicki, Michael S. Firstenberg, James P. Orlando and Thomas J. Papadimos

Contributors

Alan H Remde, Thomas Wojda, Stephen DeTurk, Tracy Butryn, Vikas Yellapu, Alyssa Green, Parampreet Kaur, James Dalkiewicz, Nguyet-Cam Lam, Elspeth Black, Andrew King, Simiao Li-Sauerwine, Steven Allen, Amanda Cooper, Roderick Quiros, Sofya Asfaw, Bess Connors, Charlotte Horne, Valery Vilchez, Stanislaw P. Stawicki, Jay Yanoff

๑) The Editor(s) and the Author(s) 2019

The rights of the editor(s) and the author(s) have been asserted in accordance with the Copyright, Designs and Patents Act 1988. All rights to the book as a whole are reserved by INTECHOPEN LIMITED . The book as a whole (compilation) cannot be reproduced, distributed or used for commercial or non-commercial purposes without INTECHOPEN LIMITED's written permission. Enquiries concerning the use of the book should be directed to INTECHOPEN LIMITED rights and permissions department (permissions@intechopen.com).

Violations are liable to prosecution under the governing Copyright Law

\section{(cc) BY}

Individual chapters of this publication are distributed under the terms of the Creative Commons Attribution 3.๑ Unported License which permits commercial use, distribution and reproduction of the individual chapters, provided the original author(s) and source publication are appropriately acknowledged. If so indicated, certain images may not be included under the Creative Commons license. In such cases users will need to obtain permission from the license holder to reproduce the material. More details and guidelines concerning content reuse and adaptation can be found at http : //www . intechopen . com/copyright-policy . html.

\section{Notice}

Statements and opinions expressed in the chapters are these of the individual contributors and not necessarily those of the editors or publisher. No responsibility is accepted for the accuracy of information contained in the published chapters. The publisher assumes no responsibility for any damage or injury to persons or property arising out of the use of any materials, instructions, methods or ideas contained in the book.

First published in London, United Kingdom, 2019 by IntechOpen

IntechOpen is the global imprint of INTECHOPEN LIMITED, registered in England and Wales,

registration number: 11086078 , The Shard, 25th floor, 32 London Bridge Street

London, SE19SG - United Kingdom

Printed in Croatia

British Library Cataloguing-in-Publication Data

A catalogue record for this book is available from the British Library

Additional hard and PDF copies can be obtained from orders@intechopen.com

Contemporary Topics in Graduate Medical Education

Edited by Stanislaw P. Stawicki, Michael S. Firstenberg, James P. Orlando and Thomas J. Papadimos p. cm.

Print ISBN 978-1-83962-238-0

Online ISBN 978-1-83962-239-7

eBook (PDF) ISBN 978-1-83962-240-3 


\section{We are IntechOpen, \\ the world's leading publisher of Open Access books}

\section{Built by scientists, for scientists}

\section{$4,300+$}

Open access books available

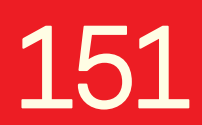

Countries delivered to

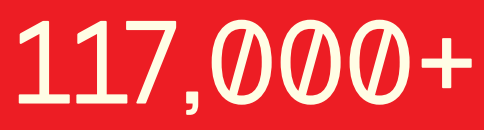

International authors and editors
$130 \mathrm{M}+$

Downloads

Our authors are among the

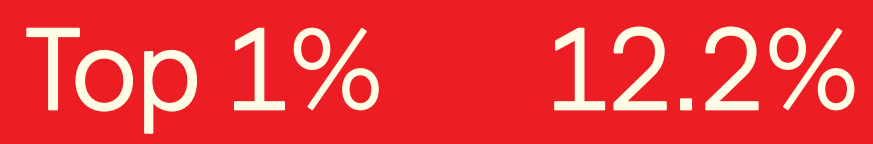

most cited scientists

Contributors from top 500 universities

\section{Interested in publishing with us? \\ Contact book.department@intechopen.com}

Numbers displayed above are based on latest data collected.

For more information visit www.intechopen.com 



\section{Meet the editors}

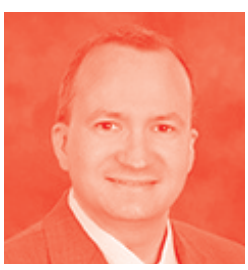

Dr. Stanislaw P. Stawicki, MD, MBA, FACS, FAIM is Chair of the Department of Research of Innovation, St. Luke's University Health Network, Bethlehem, Pennsylvania. He is Associate Professor of Surgery at Temple University School of Medicine. Dr Stawicki co-authored $>560$ scholarly publications, edited $>15$ books, and is a member of multiple editorial boards. He is an expert in clinical research, health security, medical education, patient safety, traumatology and surgical critical care. He has given numerous scientific presentations on three continents and is Board Certified in General Surgery, Surgical Critical Care, and Neurocritical Care.

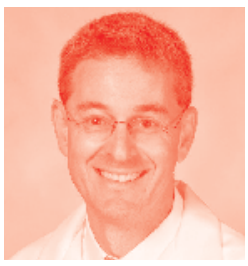

Dr. Michael S. Firstenberg, MD, FACC, FAIM is a board-certified thoracic surgeon practicing adult cardiac surgery at The Medical Center of Aurora (Colorado, USA) where he serves at the Chief of Cardiothoracic and Vascular Surgery. He currently holds Adjunct appointments in the Colleges of Medicine and Graduate Studies at Northeast Ohio Medical University and serves on the teaching faculty at the Rocky Vista University. He attended Case Western Reserve University Medical School, received his General Surgery training at University Hospitals in Cleveland, and completed a Fellowship in Thoracic Surgery at The Ohio State University. He also obtained advanced training in heart failure surgical therapies at The Cleveland Clinic. He is an active member of the Society of Thoracic Surgeons (STS), American Association of Thoracic Surgeons (AATS), the American College of Cardiology (ACC), and the American College of Academic International Medicine (ACAIM - for which he is a Founding Fellow). He currently serves a Chair of the American College of Cardiology Credentialing and Member Services Committee as well as being active on several other national society committees. He is the author of well over 200 peer-reviewed manuscripts, abstracts, and book chapters. He has Edited several textbooks on topics ranging from Medical Leadership, Patient Safety, Endocarditis, and Extra-corporeal Membrane Oxygenation - all of which include topics that he has lectured on world-wide..

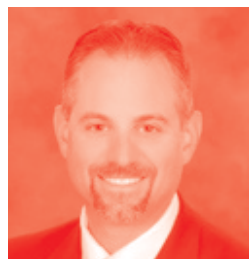

Dr. James P. Orlando, EdD serves as the Chief Medical Education Officer and ACGME Designated Institutional Official (DIO) at St Luke's University Health Network in Bethlehem, PA. He earned his Doctor of Education (Ed.D) in Corporate Learning Leadership from University of Pennsylvania, Graduate School of Education and The Wharton School. He earned his Masters of Education (M.Ed.) in Organizational Development from Temple University and two Bachelor of Art (B.A) degrees from Moravian College in International Management and American Political Thought. Dr. Orlando serves as an adjunct professor at University of Pennsylvania Graduate School of Education and Moravian College. He is an Associate Certified Coach (ACC) by the International Coaching Federation. In addition, Dr. Orlando serves on two national boards, the Accreditation Council for Graduate Medical Education (ACGME) Institutional Review Committee and on the Alliance of Independent Academic Medical Centers (AIAMC) Board of Directors. 


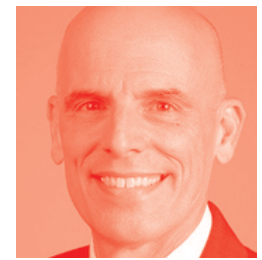

Dr. Thomas J. Papadimos, MD, MPH, FCCM, FAIM is the Professor and Associate Dean, as well as the Medical Director, Lloyd A. Jacobs Interprofessional Immersive Simulation Center Director, Division of Critical Care Medicine, Department of Anesthesiology The University of Toledo College of Medicine and Life Sciences Toledo, Ohio. Dr. Papadimos co-authored hundreds of high impact publications and co-edited books on patient safety and medical ethics. His current leadership role includes active involved in undergraduate, postgraduate, and graduate medical education. He is among top leaders in immersive medical simulation. 


\section{Contents}

Preface

Section 1

Introductory Remarks

Chapter 1

Introductory Chapter: A Quest to Transform Graduate Medical

Education into a Seamless Journey toward Practice Readiness

by Stanislaw P. Stawicki, Michael S. Firstenberg, James P. Orlando

and Thomas J. Papadimos

Section 2

Invited Commentary on Leadership in Graduate Medical Education

Chapter 2

Leadership in Graduate Medical Education

by Jay $M$. Yanoff

Section 3

Main Contents

Chapter 3

Curriculum Development: Foundations and Modern Advances in

Graduate Medical Education

by Simiao Li-Sauerwine and Andrew King

Chapter 4

Resident Autonomy

by Amanda Cooper and Steven Allen

Chapter 5

Teaching Balanced Patient Care Using Principles of Reductionism and Holism: The Example of Chronic Low Back Pain

by Alan Remde, Stephen DeTurk and Thomas Wojda

Chapter 6

Physician Burnout

by Bess Connors, Charlotte Horne, Valery Vilchez and Sofya Asfaw 
Chapter 7

Wellness in Residency: A Paradigm Shift

by Roderick M. Quiros and Elspeth Black

Chapter 8

Implementing Wellness Curriculum in Residency

by Nguyet-Cam V. Lam and Elspeth Black

Chapter 9

The Importance of Post-Doctoral Program to GME in an Academic Medical Center

by Tracy L. Butryn, Parampreet Kaur, Vikas Yellapu, Alyssa Green and James Dalkiewicz 


\section{Preface}

Graduate medical education (GME) is a vast, highly complex, and controversial topic, with numerous subtleties, nuances and intricacies. As implied in its very definition, GME's primary goal is to oversee the development of competent physicians, with the intent of ensuring health-care continuity, quality, and value well into the future. Key challenges facing GME today include increasing regulatory burden, spiraling health-care costs, demographic trends, looming shortage of providers, physician burnout, and rapidly evolving consumer expectations, among other issues.

In addition to the increasing complexity and demands of our modern GME systems, important demographic and population trends place further strain on our current and future physician workforce. The expected growth of the geriatric population segment, coupled with the system-wide demand for high-quality, safe and affordable health-care, create both a challenge and an opportunity to transform how we diagnose and treat patients. Graduate medical education is one of the critical components of this ongoing transformation.

This inaugural tome of a multi-volume collection, titled Contemporary Topics in Graduate Medical Education, provides an excellent introduction to many of the topics directly relevant to modern GME theory and operations. Beginning with an invited commentary on leadership in graduate medical education by Professor Jay M. Yanoff, the book goes on to discuss a broad range of contemporary issues encountered by GME programs across the world. These topics include curriculum development, resident autonomy issues, physician burnout and wellness, postdoctoral research programs, and finally an important discussion on reductionism and holism in the context of balanced patient care education.

More than 20 authors contributed to this book, representing an impressive group of academics, from some of the top teaching hospitals in the United States. And while the book itself represents a collection of chapters, each chapter illuminates its unique perspective across the wealth of topics discussed throughout the first volume of the cycle. The diversity of academic views and opinions is one of the key strengths of this inaugural tome. Consequently, the Editors intend to ensure that this focus on excellence continues throughout the entire series.

As the Editors of Contemporary Topics in Graduate Medical Education, we hope that the current compilation will not only provide the reader with superb value and knowledge, but also help establish the current book cycle as an important source of 
cutting edge information in this dynamically evolving and critically important area of expertise within the health-care sector.

Stanislaw P. Stawicki, MD, MBA and James P. Orlando, EdD

St. Luke's University Health Network,

USA

Michael S. Firstenberg, MD

The Medical Center of Aurora,

USA

Thomas J. Papadimos, MD, MPH

University of Toledo Medical Center,

USA 
Section 1

\section{Introductory Remarks}





\title{
Introductory Chapter: A Quest to Transform Graduate Medical Education into a Seamless Journey toward Practice Readiness
}

\author{
Stanislaw P. Stawicki, Michael S. Firstenberg, \\ James P. Orlando and Thomas J. Papadimos
}

\section{Introduction}

Graduate medical education (GME) represents the transition between the traditionally understood world of "undergraduate education," including the medical school, and the realm of fully professional engagement within a medical or surgical specialty $[1,2]$. Due to the relative shielding of trainees from full spectrum exposure to "real-life practice" environments and responsibilities, important gaps in readiness exist $[3,4]$. In response, residency and fellowship programs are undergoing significant transformation to more effectively prepare graduates to meet the expectations of a busy, modern day clinical practice $[2,5]$. As part of this transition, contemporary GME's character has evolved beyond a well-established repertoire of didactic techniques, increasingly embracing stateof-the-art immersive simulation, modern multimedia platforms, and real-time feedback technologies $[6,7]$.

This once-in-a-generation transformation is taking place right now and can be characterized by the words of Wayne Gretzky, nicknamed “The Great One," one of the greatest stars in the history of the National Hockey League. Gretzky once said, "I skate to where the puck is going to be, not to where it has been" [8]. These famous-yet very simple-words from one of hockey's most successful players of all time sum up the theme of Contemporary Topics in Graduate Medical Education in a nutshell, as our editorial team and chapter authors share their latest thoughts and wisdom on preparing the next generation of physicians. We see that the nature of physicians' work has changed immensely over the past 2-3 decades. Yet, the currents underlying this change will present different-and truly unique-opportunities and challenges for tomorrow's physicians, requiring an in-depth rethinking of the concept of "readiness for (independent) practice" [9].

The abovementioned challenges are clearly evident when examining contemporary healthcare practices and outcomes. For example, mortality from cancer in high-income countries has declined over the last 10 years due to better prevention, early detection, and improvements in treatment $[10,11]$. Still, there are approximately 40 million adult smokers in the United States, with an estimated annual cancer-attributed mortality of approximately 33\% [12]. Furthermore, while the average annual growth of per capita health expenditures has declined during the past decade, the US healthcare system features the highest per capita 
cost (approximately $\$ 10,822$ in 2017) among developed nations [13]. Interestingly, unmet behavioral health needs are among the top five conditions that drive overall healthcare costs [14]. How are we preparing future physicians to provide care in a system that treats symptoms rather than underlying problems while providing that care in a way that improves quality and limits costs? Many of the answers are provided in a chapter by Remde, et al., on "Teaching Balanced Patient Care using Principle of Reductionism and Holism" [15] and a superb contribution by Li-Sauerwine and King, titled "Curriculum Development: Foundations and Modern Advances in Graduate Medical Education [16]." In addition, the chapter by Butryn et al. [17] discusses the value of Postdoctoral Research Program as a unique value proposition that is not generally well known and often underappreciated despite the potential for substantial positive impact on institutions, trainees, and a broad range of postgraduate educational programs.

As one explores the different chapters within Contemporary Topics in Graduate Medical Education, it becomes apparent how complex, interconnected, and interdependent various components are within the matrix of modern healthcare. The same can be said about the different domains of professional life. After all, a physicianin-training is an individual with a personal life, family, goals and ambitions, as well as unique gifts and abilities. During their training, residents and fellows experience tremendous amounts of personal and professional stress, and because it is virtually impossible to separate the "clinical life" from the "home life," concepts such as burnout, resilience, and work-life integration (as opposed to the increasingly outdated paradigm of work-life balance) begin to emerge $[18,19]$. The latter paradigm, centered around satisfactorily reconciling and integrating work and non-work domains [18], is especially important in the context of training "practiceready" physicians in the era where our daily routines are increasingly defined by the pervasiveness of technological advances and trends [20].

One particularly strong riptide that will place increasing amounts of stress on the entire healthcare system (e.g., institutions, providers, insurers, and patients) is the imminent healthcare workforce shortage [21]. It has been estimated by the Association of American Medical Colleges (AAMC) that by 2025 there will be a deficit of between 14,900-35,600 primary care physicians and 37,400-60,300 nonprimary care specialists [22]. This is especially concerning since countries with the best-performing healthcare systems (as measured by longevity, infant mortality, and patient satisfaction) tend to have the highest percentage of family physicians [23]. Many national, state, and local initiatives are underway to address these upcoming shortages. Specifically, the Association of Departments of Family Medicine (ADFM) released strategies for increasing the primary care workforce, including development of more targeted high school/college pipeline programs, medical training innovations, office practice transformation, and payment reforms [24]. One of ADFM's strategies is to equip residents with better wellness skills to manage stress and increase physician work/life satisfaction and joy of medicine as the demand for their services increases. A number of chapters in this book address the interrelated topics of physician burnout (by Connors et al. [25]), wellness (by Lam et al. [26] and Quiros et al. [27]), and resident autonomy and graduated responsibility (by Cooper and Allen [28]), effectively acting as lighthouses for institutional leaders and residency program directors navigating the stormy seas of contemporary GME transformation. It is hoped that improved awareness and better knowledge will help facilitate the gradual transition in our collective mindset, moving away from "burnout" while pursuing "wellness" [29]. Another critically important area of graduate medical education - curriculum development-is discussed by Li-Sauerwine and King [16], in an outstanding chapter that touches upon both foundational aspects and recent advances in this domain. Taken together, these contributions provide 
insight into some of the latest thinking about what independent medical and surgical practice may look like 10-20 years from now.

Finally, this compendium would not be complete if we did not discuss the importance of leadership in medical education at academic medical centers, including the rapidly evolving theoretical considerations and their practical applications. The editors are proud to introduce Dr. Jay Yanoff, Professor of Otolaryngology-Head and Neck Surgery - at Drexel University College of Medicine in Philadelphia, as the author of the Opening Chapter for the Contemporary Topics in Graduate Medical Education book series. A true expert and academician, Dr. Yanoff is the former associate dean of graduate medical education and former designated institutional official (DIO) of Hahnemann University Hospital in Philadelphia, Pennsylvania. He is nationally recognized for his work in how the mind functions during the learning process and is the co-author of numerous textbooks, book chapters, and articles in refereed journals. In the Introductory Chapter, Dr. Yanoff reflects on his leadership experiences and provides a road map for effective leadership in academic medical centers based on more than $40+$ year-long academic career [30].

Addressing the topic of leadership in medicine is extremely important because, as recognized by Mathis, “There is nothing in a physician's education and training that qualifies him to become a leader" [31]. The physician's primary purpose and professional duty is the care of our patients, accompanied by the education of patients, students, residents, colleagues, and politicians, fostering the growth of knowledge. However, in doing so we bear the weight of social responsibility as it relates to not only individual healthcare but also the public's general health and well-being. The "space of appearance" of physicians puts them at the heart of patient safety, efficacious delivery of care, and financial stewardship of resources. To reconcile these critical duties and responsibilities is certainly not an easy task. Moreover, the modern physician may be required to cope with conflicting priorities and demands, such as the need of consistently demonstrating high levels of emotional intelligence and empathy while struggling with empathy-depleting burnout [32-36].

While this inaugural tome of Contemporary Topics in Graduate Medical Education represents a tremendous effort by many leaders in the field of graduate medical education, by no means is it intended to be the final word on the topic. In fact, this volume will hopefully serve as a foundation for further texts in this area as educators continue to explore the general question of "how do we train doctors of the future?" The challenges are great as the goals and educational landscape continue to evolve-as do many other areas of medicine. As mentioned in previous paragraphs, being a physician is not limited to providing healthcare services or medical/administrative leadership, but extends well beyond these "technical" functions. After all, and before all other purposes, a physician is an individual who is responsible for the health and well-being of others-our patients. How do we instill the traditionally understood sense of "ownership" regarding patient outcomes? How do we build and foster a sense of accountability without consequences being perceived as punishment? These concepts, especially in the ethical foundation of "what we do" as our core business, permeate throughout all of the topics in this text, and unless we find ways of identifying individuals who have such innate sense of caring and responsibility, a fundamental question remains_- "how do we teach it?" Furthermore, as more and more areas of medicine evolve into team-based and multidisciplinary models of care, how do we train physicians to not only lead, but embrace, such models [37-40]? Effective multidisciplinary teams require strong yet flexible leaders who possess a solid foundation in the scientific literature and a broad understanding of complex disease states and pathophysiology, medical economics, ethics, and building and maintaining collaborative relationships across disciplines. Physicians are uniquely suited to provide such leadership, and it is critical for our 
graduate medical education systems to adequately prepare such leaders of tomorrow. The task is not easy, but hopefully texts such as this can help make it easier.

The medical practitioner of the future must embrace the greatest degrees of flexibility, nimbleness, and adaptability. Our medical education system, and GME in particular, must effectively adapt and evolve with the times because change is a continuous state of this noble profession. Heraclitus, a sixth century BC Greek philosopher once said, "There is nothing permanent except change" [41]. And this was very much echoed by Charles Darwin who very eloquently noted that "It is not the strongest of the species that survives, nor the most intelligent, but the one most responsive to change" [42]. The US healthcare system is no different, and by proxy neither is graduate medical education. As we look to the challenges and opportunities ahead of us in medical education, this book provides the wisdom, tools, and resources to effectively respond to change so that we can better prepare physicians for where the puck is going!

\section{Author details}

Stanislaw P. Stawicki ${ }^{1 *}$, Michael S. Firstenberg ${ }^{2}$, James P. Orlando ${ }^{3}$ and Thomas J. Papadimos ${ }^{4}$

1 Department of Research and Innovation, St. Luke's University Health Network, Bethlehem, PA, USA

2 Department of Cardiothoracic Surgery, The Medical Center of Aurora, Aurora, CO, USA

3 Department of Medical Education, St. Luke's University Health Network, Bethlehem, PA, USA

4 Department of Anesthesiology, The Ohio State University College of Medicine, Columbus, OH, USA

*Address all correspondence to: stawicki.ace@gmail.com

\section{IntechOpen}

(C) 2019 The Author(s). Licensee IntechOpen. This chapter is distributed under the terms of the Creative Commons Attribution License (http://creativecommons.org/licenses/ by/3.0), which permits unrestricted use, distribution, and reproduction in any medium, provided the original work is properly cited. (cc) BY 


\section{References}

[1] Cruess RL, Cruess SR, SteinertY. Teaching Medical Professionalism:

Supporting the Development of a Professional Identity. England: Cambridge University Press; 2016

[2] Ricketts TC, Fraher EP.

Reconfiguring health workforce policy so that education, training, and actual delivery of care are closely connected. Health Affairs. 2013;32(11):1874-1880

[3] Blinman TA. Competency Based Medical Education is Wrong for Surgery. 2017. Available from: https://osf.io/ kzrqp/download?format=pdf [Accessed: April 22, 2019]

[4] Franzone JM et al. Progressive independence in clinical training: Perspectives of a national, multispecialty panel of residents and fellows. Journal of Graduate Medical Education. 2015;7(4):700-704

[5] Bhatti NI, Ahmed A. Improving skills development in residency using a deliberate-practice and learnercentered model. The Laryngoscope. 2015;125:S1-S14

[6] Seidman RH. Serious Games: The Confluence of Virtual Reality, Simulation \& Modeling, and Immersive Education. 2009. Available from: https://academicarchive.snhu.edu/ bitstream/handle/10474/3121/ sabbatical2009seidman_pt2. pdf? sequence $=2$ [Accessed: April 22, 2019]

[7] Stawicki TT et al. From "pearls" to "tweets:" How social media and webbased applications are revolutionizing medical education. International Journal of Academic Medicine. 2018;4(2):93

[8] Tallent E. "I Skate to Where the Puck Is Going To Be, Not Where it Has Been"-Musings on gut decisions and our future. Internet Reference Services Quarterly. 2011;16(1-2):1-7

[9] Nasca TJ et al. The next GME accreditation system-Rationale and benefits. New England Journal of Medicine. 2012;366(11):1051-1056

[10] García-Esquinas E et al. Impact of declining exposure to secondhand tobacco smoke in public places to decreasing smoking-related cancer mortality in the US population. Environment International. 2018;117:260-267

[11] Bray F et al. Global cancer statistics 2018: GLOBOCAN estimates of incidence and mortality worldwide for 36 cancers in 185 countries. CA: A Cancer Journal for Clinicians. 2018;68(6):394-424

[12] Bakalar N. Cancer Deaths Continue a Steep Decline. 2018. Available from: https://www.nytimes.com/2018/01/05/ science/cancer-deaths-decline.html [February 8, 2019]

[13] Henry_J_Kaiser_Family_ Foundation. Health Costs. 2019. Available from: https://www.kff.org/ health-costs/ [February 8, 2019]

[14] Loeppke R et al. Health and productivity as a business strategy: A multiemployer study. Journal of Occupational and Environmental Medicine. 2009;51(4):411-428

[15] Remde A, DeTurk S, Wojda T. Teaching balanced patient care using principles of reductionism and holism: The example of chronic low back pain. In: Contemporary Topics in Graduate Medical Education. London, England: IntechOpen; 2018

[16] Li-Sauerwine S, King A. Curriculum development: Foundations and modern advances in graduate medical education. 
In: Contemporary Topics in Graduate Medical Education. London, England: IntechOpen; 2019

[17] Butryn T, Kaur P, Yellapu V, Green A, Dalkiewicz. The importance of post-doctoral program to GME in an academic medical center. In: Contemporary Topics in Graduate Medical Education. London, England: IntechOpen; 2019

[18] Valcour PM, Hunter LW. Technology, Organizations, and Work-Life Integration. In: Kossek EE, Lambert SJ, editors. Work and Life Integration - Organizational, Cultural, and Individual Perspectives. Mahwah, New Jersey: Lawrence Erlbaum Associates; 2005

[19] Shanafelt TD et al. Changes in burnout and satisfaction with work-life balance in physicians and the general US working population between 2011 and 2014. Mayo Clinic Proceedings. 2015;90:1600-1613

[20] Yoo Y et al. Organizing for innovation in the digitized world. Organization Science. 2012;23(5):1398-1408

[21] Sharp M. The Impact of the Affordable Care Act on the Impending Doctor Shortage: A Prospective Analysis. 2016. Available from: https://open. bu.edu/bitstream/handle/2144/17052/ Sharp_bu_0017N_12033.pdf?sequence=1 [Accessed: April 22, 2019]

[22] AAMC. New Research Confirms Looming Physician Shortage. 2016. Available from: https://www.aamc.org/ newsroom/newsreleases/458074/2016_ workforce_projections_04052016.html [February 8, 2019]

[23] Starfield B. Primary care and health: A cross-national comparison. JAMA. 1991;266(16):2268-2271

[24] ADFM. Association of Departments of Family Medicine Strategic
Committees. 2017. Available from: https://adfm.org/media/1424/infoabout-adfm-strategic-committees.pdf [February 8, 2019]

[25] Connors B, Horne C, Vilchez, Asfaw S. Physician burnout. In: Contemporary Topics in Graduate Medical Education. London, England: IntechOpen; 2019

[26] Lam NC, Black E. Implementing wellness curriculum in residency. In: Contemporary Topics in Graduate Medical Education. London, England: IntechOpen; 2019

[27] Quiros RM, Black E. Wellness in residency: A paradigm shift. In: Contemporary Topics in Graduate Medical Education. London, England: IntechOpen; 2019

[28] Cooper A, Allen S. Resident autonomy. In: Contemporary Topics in Graduate Medical Education. London, England: IntechOpen; 2019

[29] Eckleberry-Hunt J et al. Changing the conversation from burnout to wellness: Physician well-being in residency training programs. Journal of Graduate Medical Education. 2009;1(2):225-230

[30] Yanoff JM. Leadership in graduate medical education. In: Contemporary Topics in Graduate Medical Education. London, England: IntechOpen; 2019

[31] Mathis LL. The Mathis Maxims: Lessons in Leadership. Houston, Texas: Leadership Press; 2001

[32] Hojat M. Empathy in Patient Care: Antecedents, Development, Measurement, and Outcomes. Cham, Switzerland: Springer Science \& Business Media; 2007

[33] Adolph MD et al. Palliative critical care in the intensive care unit: A 2011 perspective. International Journal of 
Critical Illness and Injury Science. 2011;1(2):147

[34] Tolentino JC et al. What's new in academic medicine: Can we effectively address the burnout epidemic in healthcare? International Journal of Academic Medicine. 2017;3(3):1

[35] Uchino R et al. Focus on emotional intelligence in medical education: From problem awareness to system-based solutions. International Journal of Academic Medicine. 2015;1(1):9

[36] DeCaporale-Ryan L et al. The undiagnosed pandemic: Burnout and depression within the surgical community. Current Problems in Surgery. 2017;54(9):453-502

[37] Tolentino JC et al. Introductory chapter: Developing patient safety champions. In: Vignettes in Patient Safety. Vol. 2. Rijeka, Croatia: IntechOpen; 2018

[38] Holmes DR et al. The heart team of cardiovascular care. Journal of the American College of Cardiology. 2013;61(9):903-907

[39] McAlister FA et al. Multidisciplinary strategies for the management of heart failure patients at high risk for admission: A systematic review of randomized trials. Journal of the American College of Cardiology. 2004;44(4):810-819

[40] Bach JA et al. The right team at the right time-Multidisciplinary approach to multi-trauma patient with orthopedic injuries. International Journal of Critical Illness and Injury Science. 2017;7(1):32

[41] Haxton B. Heraclitus: Fragments. USA: Penguin Books; 2001

[42] Proykova A. Session 1: Knowledge Production and Certification. 2004. Available from: https:// www.researchgate.net/profile/
Ana_Proykova/publication/235696441_ Session_1_Knowledge_ production_and_certification/ links/09e41512aa500e67ab000000.pdf [Accessed: April 22, 2019] 

Section 2

\section{Invited Commentary on Leadership in Graduate Medical Education}





\title{
Leadership in Graduate Medical Education
}

\author{
Jay M. Yanoff
}

\begin{abstract}
Graduate medical education (GME) is a very complex endeavor within an even more complex healthcare system. This chapter examines many questions that need to be considered and the role of the key individual with oversight of the GME, the designated institutional official (DIO). Topics examined are the leadership theories, practices and strategies for the DIO, dealing with change when the DIO starts, using authority versus power, effective problem-solving and decision-making, adaptive leadership style, the historical function of the DIO, as well as the many tools available to the DIO including networking. The chapter concludes with several pearls of wisdom to positively help the DIO meet the many challenges of this very important role in GME.
\end{abstract}

Keywords: graduate medical education (GME), designated institutional official (DIO), leadership, dealing with change, problem-solving and decision-making

\section{Introduction}

Graduate Medical Education (GME) is a very complex operation within an even more complex healthcare system. In order to be an effective leader within both a complex operation and system, there are many questions that need to be considered. For example, what is the purpose of having GME programs in the organization? What are both the long term and short term goals of the institution? What are expectations and outcomes sought by having GME programs? In some cases, what is the relationship between the hospital doing the training and any medical school who has oversight of the education? Since there are often multiple personalities with whom one must work, who are the leaders and participants and what are their values and expectations? In this complex environment, what are other relationships where you must interact such as accrediting bodies, affiliated institutions and other training institutions in your area? These questions stem from the very origins of developing any curriculum and plan for instruction and should be used as starting points to be a successful educational leader [1]. In order to be a successful leader in today's GME world, one must first recognize that this is not a simple role and requires skills, knowledge and the right temperament to deal with a multitude of issues.

\section{The role of the designated institutional official (DIO)}

In the middle of this complex system is the role of the designated institutional official (DIO). In this role, there are many individuals across the institution with whom the DIO interacts. Organizationally, the DIO is responsible for reporting to 
the Board of Directors of the institution (generally, a hospital), at least one medical school, hospital administration including the Chief Executive Officer, Chief Financial Officer, Chief Human Resources Officer, accrediting body requirements (in medical education, the ACGME), as well as the Centers for Medicare and Medicaid Services (CMS) and other funding sources to name a few of the most important ones. There are also consequences when there are issues of non-compliance that can lead to reduced governmental funding, loss of accreditation, National Residency Match Program (NRMP) match violations, and low resident and faculty morale.

In terms of an organization's power system, the DIO works primarily in the middle but also flexes into various Tops, Bottoms, Middles, and Customer roles [2]. DIOs as Tops have designated responsibilities (ex. accreditation), as Bottoms experience problems that we think higher-ups should take care of (ex. residency clinic operations), as Middles experience competing demands and priorities (ex. service versus education), and as Customers when we are looking to another department for a service we need to move our work ahead (ex. HR support to onboard new trainees). The DIO constantly toggles in and out of Top, Middle, Bottom, and Customer roles. In each of these roles, there are unique opportunities for contributing to organizational effectiveness and pitfalls that readily lead us to forfeit those contributions. The DIO's job within an organization's power system is to recognize and mitigate common reflex responses such as taking on too much responsibility when we are the Top, holding higher-ups too responsible when we are the Bottom, losing our connectivity with other parts of the organization when we are the Middle, and not participating enough in improving internal service delivery when we are the Customer [2].

In terms of oversight, there are many who have reporting relationships with the DIO including Program Directors of each internship, residency and fellowship program, Program Coordinators, DIOs at both sending and receiving institutions, oversight of the office of GME and most importantly, the education and training of interns, residents and fellows. Furthermore, the DIO is responsible for the Graduate Medical Education Committee (GMEC), the Clinical Learning Environment, and multiple other responsibilities demanded by the accrediting bodies including completion of affiliation agreements, compliance with work hours, initiating and maintaining wellness programs, encouraging and supporting scholarly activity, oversight of moonlighting, continual review of the educational programs, identifying "red flags" and then addressing the concerns, and any citations with proactive strategies and action plans. Needless to say, the role of GME leader in this very complex "middle person" system requires much knowledge, strong interpersonal skills and highly ethical values to be successful.

After many years serving as the Associate Dean for Graduate Medical Education at a medical school, Vice President for Academic Affairs at a hospital, and Chief Graduate Medical Education Officer (DIO) for the last 18 years at a medical school/ hospital, I want to reflect on those theories, practices and strategies that I have utilized which may help others who are in this complex "middle person" leadership role.

\section{Leadership theories, practices and strategies}

My definition of leadership is followership. Unless we can get others to follow, we cannot lead. Goffee and Jones coined the phrase, "Authentic Followership" explaining that leaders need to convey Authenticity, Significance, Excitement, and Community to followers in order to truly lead [3]. I believe that people want to follow someone who is "authentic," someone who they can trust, someone who will actively listen and hear their issues, and someone who believes in action, not just 
words. True leadership takes time to develop and is not accomplished overnight. Flexibility is a key component to building trust and caring relationships with those with whom we interact.

One of the first questions to think about is what if you are new at an institution and are entering for the first time. I believe that you need to look at three levels of maturity: (1) your experience, professional background and leadership ability, (2) the institution's maturity, and (3) the employees experience and background with whom you will be working. Analyze how experienced you are in the DIO role. Ask some of the following: have you been in this role before? What was the size of the institution? How experienced do you feel? Regarding the institution: what is its historical background? How much does the administration of the institution know about GME and what is their support? How long have they had GME programs? What is the accreditation status of the programs? And finally, regarding the people at the institution: what is the maturity of the individuals in the Office of GME? What is the range of experience of the Program Directors and Coordinators? What do they expect from their leader?

There are several key thoughts here. Let us assume you have been in GME for many years and are considered mature. If so and you are entering a new system, you can take a directive role of leadership because you are the most mature. However, that is rarely the case. In most cases, the leader is entering a mature system, one that has been in existence for a long period of time with the employees and Program Directors already in place. The leader is a newcomer to the system irrespective of his/her maturity. In that case, the leader has to enter very gently. The leader needs to meet with key people, listen carefully, ask many questions, assess their needs, and ask what they expect from you and how you can help them. If possible, you may want to meet with the person who preceded you to learn as much as you can about the system from that person's perspective. Note, when coming into a new system, it is important to ingratiate yourself to those around you until such time as you can gain the respect of those with whom you will be leading.

\section{Dealing with change}

I also believe that organizations, like people, have difficulty dealing with change. Organizational management experts note that basically, people resist change [4]. The greater the change, the stronger the resistance. Change is inevitable, but so is the resistance. Thus, if you are coming into a new institution, you must be prepared for the reaction to you. As the new person, there is an expectation that there will be change. I believe that organizations go through the same stages of change similar to those described by Elisabeth Kubler-Ross when referring to those experiencing death and dying. The stages are (1) denial, (2) anger, (3) bargaining, (4) depression and finally (5) acceptance [5]. It is reasonable to expect these earlier stages will occur with a new DIO entering an institution before there is acceptance of his/her role, responsibilities and actions.

As the middle person in a complex system, where do we start. I have learned several things in my career. In a previous role, I sent out a questionnaire to many individuals that would take 5 minutes to complete. None were returned to me. In frustration, I called and asked if I could come to speak with them for a few minutes. In every case, the person said yes and the conversations often lasted an hour with much valuable information gained. What I leaned from this was to go to their space where they are comfortable, secure and open. Another example comes from early in my career when I was a 27 year old principal of an elementary school. Being young, inexperienced and in a role of responsibility, I developed a philosophy of 
what I called “management by movement.” I made sure I was in every classroom, every day. I believe that even though it is more comfortable to sit in our office and wait for issues to come to us, I am amazed with what gets accomplished by going to other people's space. By doing so, this facilitates building bridges and leads to more long-lasting relationships in the future. They are more comfortable and willing to share in their area than what may feel as intimidating when in my office/space. I use this strategy even to this day.

\section{Using authority versus power}

As a leader, I like to think of the difference between power and authority. Power is what you can make people do. Authority is what you can get people to do. Power is authoritarian and while it is needed sometimes, it often yields resentment and anger. I rarely ever wanted to use power to get things done, however when I had a trainee with drug or alcohol issues, it needed interventions immediately. I had to be more assertive and pull the person from service in order to get the individual the required assistance as soon as possible. However, in most cases, I want to use authority to get things done. I always want to base my relationships on trust, honesty, fairness and compassion to accomplish my goals and objectives and using power rarely yields this outcome.

\section{Effective problem-solving and decision-making}

A major aspect of leadership is problem-solving and decision-making. I often call upon Dewey's four stage model of problem-solving: (1) problem sensitivity (2) problem formulation (3) search and (4) resolution [6]. Problem sensitivity is the recognition that there is a problem in the first place. Problem formulation to me is the most important. Clearly determine what is the question being asked. If this is not articulated specifically in the beginning, we will search and not find the correct solution. It is important to make sure that the right question is being asked or an incorrect solution will be found and not be useful. If the problem formulation is carefully articulated, then a search can be made of alternatives and here is where I use a different model. I decide whether I want to maximize or satisfice. If I maximize, I want to generate as many options as possible and choose the best one. This takes time and should be used when making the most profound decisions. Some examples include:

1. A program has lost some of its faculty to another institution. Should we voluntarily withdraw the program and orphan the trainees or attempt to find new faculty to continue the program?

2. We have interviewed several people for the Program Director role. Each has different strengths and weaknesses. Which one shall we choose?

Satisficing is generating options and deciding on the first satisfactory one. An example: we want to have a luncheon for the house staff. What kind of food should we order? The real issue here is deciding at the beginning if we want to maximize or satisfice. Many people make the mistake of maximizing on issues where they should satisfice and visa-versa.

As leaders, we are continually called upon to make decisions and solve problems. As a leader, I use the Dewey model in three ways-how I solve problems, what I expect from employees and how I deal with problem trainees. 
For me as the leader, I first try to articulate the problem as carefully as possible. I then determine if this requires maximizing or satisficing. I generate alternatives. If maximizing, I want as many alternatives as possible and want to pick the best solution. If satisficing, I accept the first alternative and move on. Leaders are best served who can differentiate and use these problem-solving skills. It will save much time and energy and yield better results.

When I first met new employees, I often shared this model with them. I indicated that I want them to think independently and want them to bring me problems they cannot solve themselves. So, when they came to me with a problem, I would ask them to articulate their thinking. I would then ask the person to tell me his/her resolution and invariably, the solution was almost always exactly what I would have selected. Both of us walk away being very satisfied with the process and the conclusion.

When I was dealing with trainees facing difficult situations and challenges, I tried very carefully to listen to what was the issue. I found several keys that I used in finding the problem resolution. The first step was, were they blaming others or were they taking some responsibility for the problem? If they were blaming others, I realized I could do little to help them. However, if they were seeing themselves as some part of the problem, I might be of some help. The second step was if they were taking some responsibility, were they willing to do something about it? If so, I could be of help.

A former Dean for whom I worked called upon me for what he considered his most difficult problems. He indicated that after I investigated a problem (I used the Dewey method noted above), I was always "honest and fair." I think this is an important learning as a leader in GME that we are honest, fair and compassionate and certainly do not play favorites.

\section{Adaptive leadership style}

It is important to note that no leadership style is best. I believe the best leaders are those who are able to evaluate the situation, be adaptive and use the appropriate style for the situation. I always tried to understand the styles of those above me and below me and adapt accordingly. An example-While I am an individual who thought about what I wanted in a meeting with my superior, I would prepare my rationale in advance in order to lead into my conclusion. However, when I met with him, he would constantly say, “Get to the point.” I finally learned that I would present my conclusion first and if he wanted the particulars, I already had them prepared. Since there was a trust level between us, he would often accept my conclusion without an explanation. The lesson here is that we may have to adapt our style to meet the expectations of others if we want the positive results we desire. In other words, try to understand the dynamics between you and the other person and then work with it not against it.

I think that a leader must show high moral and ethical values. I think we all want to work with people like ourselves. How do we know if a person is like ourselves? I do not believe that it is one's gender, race, etc., but their values. When I interviewed a potential candidate, I ask the following: "Tell me about yourself." I am quiet and wait for an answer. I am not looking for what already is listed on his/her resume but rather any value words that I hear. If the candidate has difficulty articulating, I then ask them to give me 10 words that their best friend would use to describe them. I think we all want to work with people who have our same high values. Good team members are those individuals who have the same common goals and values.

Because the role of the DIO is so complex with multiple interrelationships, many responsibilities, time lines and expectations, time management is critical. Consider priorities and try to reduce chaos in the job. Obviously, we have all learned the value 
of making lists in medicine. Our minds will hold will not hold all the information we need nor the various tasks that need to be completed. Anticipate, plan ahead and complete tasks prior to the deadlines. Invariably other priorities will intercede so get things done as early as possible. This will reduce the natural stress of this very stressful job. Many years ago, I made a presentation at the Association for Humanistic Psychology entitled, "Slowing Down the Process and Learning to Ride Your Biocycle" [7]. The premise was that we have various times in the day when we are most productive and we should determine when those times are and work accordingly. I happen to be an early morning person who is less productive in late afternoon. As a result, I do my most important and creative work at those times when I feel most productive. We cannot work at maximum productivity all the time. Pick your best times and schedule the items that require your most attentive awareness during the most productive periods.

\section{Historical function of the DIO}

As a DIO, you are not alone. Years ago, there was no such role as a DIO. The ACGME simply required that every institution have "a designated institutional official" (lower case). Our own institutions did not know what that meant. However, with much pleading, the function was moved from a responsibility to a role. As such, the administrators at our institutions now know or should know what is required by this very important role. No institution can maintain its accreditation status without having a very competent individual in this very complex role.

\section{Using all the tools in your toolbox}

I grew up living above my father's hardware store. My grandfather was a very accomplished woodworker who made gavels for the United States Senate. Thus, I am a person who values tools. Tools make our lives easier. I believe that the more you use a tool, the more skilled and able you become. It is also important to use the right tool in the appropriate situation. A hammer and a screw driver do very different things. The ACGME provides many GME tools to help the DIO. There are also other tools in the market that make it easier to monitor work hours, evaluate residents, evaluate faculty, develop and maintain schedules, etc. Use them as they save time, energy and meet ACGME guidelines and requirements.

\section{Networking}

No DIO should minimize the importance of networking. As a result of our establishing a regional disaster plan, the DIOs in my area continue to meet to this day as a regional GME leadership group. There are now 23 institutions involved. Not only do we discuss the most pressing issues facing all of us, but we have contacts we can make with the DIOs of the other institutions who can help when problematic issues arise. Of course, we do not talk salary. However, we do discuss preparations for a CLER visit, how is your institution handling suicide prevention, how do you maintain confidentiality with feedback in very small programs, what is your process for the Annual Program Evaluations (APEs) and Annual Program Reports (APRs), and multiple other issues. On a regular basis, an institution will send out a query 
regarding an issue and many respond with suggestions. Not only is it important to attend the national meetings, but local networking is an excellent tool for the DIO. If you have a question, there is some DIO out there who has had that situation and is willing to help. Again, you are not alone.

While the DIO role may seem overwhelming at times, there are many exciting parts to the job. I love to think of all the practicing physicians with whom I have helped in their careers. I once estimated that there are over 6000 physicians whose certificates I have signed and the enormous number of patients for whom they provide care. I have seen some of my former trainee leaders now become Program Directors and DIOs. They now model and emulate many of the values they have learned by experiencing positive, upbeat and sensitive leadership.

\section{Impact on trainees}

In my career, I have measured impact of educational experiences. By impact, I mean that there is some external experience that changes the learner dramatically and internally. We rarely have the opportunity to know exactly the impact we are having on others. However, sometimes we are fortunate to find out. Recently, I received a call from a former student who indicated he was trying to find me because he was in the first class I taught 55 years ago. He indicated that I had made learning so much fun for him as a sixth grader that it influenced him to become a teacher, always make learning fun for his students. He indicated that he used this philosophy in teaching history, coaching and writing four books. It is nice to know that if we do the right things, sometimes we may have a major impact on those with whom we interact.

\section{Several pearls of wisdom}

There are three bits of advice I would like to share.

1. When I was in my early teens, I was required in school to learn a Rudyard Kipling poem entitled "If." To this day, I cannot forget the theme of this poem which is "If you can keep your head when all about you are losing theirs, then you my son shall be a man." Throughout the chaos that we experience being in the middle of a complex system, this quote gives me guidance and strength to keep me sane.

2. We are all achievers and want to accomplish much in the GME leadership role. One learns very quickly that everything cannot be accomplished immediately. Thus, I often remind myself of a Winston Churchill statement to Englander's during WW II, "It is better do something than nothing while waiting to do everything." As a GME leader, do not get mired in doing nothing and do what can be accomplished. Everything cannot be done at once. Start with the most important and then move on to the next task at hand.

3. Finally, while you will have wellness programs for the house staff, take care of yourself. I often advise others that "Self-care is not selfish." If you are not healthy and let a role, job or responsibilities to disable you, then you will not be able to be of worth to the organization, your family or yourself. As noted many times above, this role is complex and you must take care of you so that you can be of help to others. 


\section{Conclusion}

In conclusion, I have tried to articulate the difficult middle role that the leader in graduate medical education must play. However, if the leader uses his/her knowledge of how the system works, and employs his/her skills, appropriate tools and strong personal values, the leader will have much of the pride, meaningful highlights, and success that I have felt in my career. The subsequent chapters in this book contain substantial and practical skills, tips, examples, and research for GME leaders to improve their effectiveness and overall impact. I feel that there can be no more satisfying and fulfilling job than being a good leader in graduate medical education.

Jay M. Yanoff, Ed.D. was the Chief GME Officer and Designated Institutional Official at Drexel University College of Medicine and Hahnemann University Hospital for 18 years.

\section{Author details}

Jay M. Yanoff

Graduate Medical Education Officer, Designated Institutional Official, and Professor at Drexel University College of Medicine and Hahnemann University Hospital, Philadelphia, Pennsylvania, The United States of America

*Address all correspondence to: jay@jayyanoff.com

\section{IntechOpen}

(C) 2019 The Author(s). Licensee IntechOpen. This chapter is distributed under the terms of the Creative Commons Attribution License (http://creativecommons.org/licenses/ by/3.0), which permits unrestricted use, distribution, and reproduction in any medium, provided the original work is properly cited. (cc) BY 


\section{References}

[1] Tyler RW. Basic Principles of Curriculum and Instruction. Chicago: University of Chicago Press; 1949

[2] Oshry B. Leading Systems: Lessons from the Power Lab. 1st ed. San Francisco, Calif. [Great Britain]: Berrett-Koehler Publishers; 1999

[3] Goffee R, Jones G. Why Should Anyone be Led by you? What it Takes to be an Authentic Leader. Boston, MA: Harvard Business School Press; 2006

[4] Hodgells RM. Management-Theory, Process and Practice. Philadelphia, PA:

W.B. Saunders Company; 1975

[5] Kubler-Ross E. Questions and Answers on Death and Dying: A Companion Volume to on Death and Dying. Delran, NJ: Simon and Shuster; 2011

[6] Yanoff JM, Bryan WE. Utilizing Lewinian principles for an institutional planning process within a medical school. In: By EHS, Whelan SA, editors. The Legacy of Kurt Lewin: Field Theory in Current Practice. New York: SpringerVerlag; 1986

[7] Yanoff JM and Hoffman RG. Slowing Down the Process and Learning to Ride your Biocycle. Paper presented to the Association for Humanistic Psychology; New York; 1975 

Section 3

Main Contents 



\title{
Curriculum Development: Foundations and Modern
}

\section{Advances in Graduate Medical Education}

\author{
Simiao Li-Sauerwine and Andrew King
}

\begin{abstract}
Curriculum development has undergone many transitions since the inception of medical education in the United States in the 1800's. In this chapter, we briefly review the history of curriculum development in medical education. We discuss the landmark models of curriculum development including the concept of a curriculum map and Harden's SPICES model of educational strategy, detail the six steps of Kern's foundational framework, and provide an overview of the PRISMS strategy. We address the importance of adult learning theory and the advancing understanding of education for the millennial generation, including implementation of the flipped classroom model of education. Finally, we turn our focus on contemporary applications of curriculum design, including the application of simulation to medical education, the rise of massive open online courses (MOOC), and the implementation of free open access medical education (FOAM) within undergraduate and graduate medical curricula.
\end{abstract}

Keywords: medical education, curriculum, pedagogy, andragogy, needs assessment, evaluation, objectives, adult learning theory, flipped classroom, technology-based learning, simulation, massive open online courses (MOOC), free open access medical education (FOAM, FOAMed)

\section{Introduction}

It can be argued that the naissance of traditionally-regarded medical education in the United States began in the civil war era with for-profit "proprietary schools" which were typically less than one year in duration and the contents of which were delivered only in didactic form [1]. The Flexner report [2] in the 1920's ushered in a new era where an emphasis on lectures and textbooks in medical schools were set aside for laboratory and clinical teaching. Medical education moved from a substantive system reliant on memorization to one which was now procedural - a focus on the process itself of acquiring scientifically sound information. Between the two world wars, undergraduate medical education was further shaped into the recognizable modern form with the first two years containing preclinical education, and the second two years with clinical rotations in the major specialties. Additionally, the concept of post-medical school training took hold with the development of internships 
and residency programs. Although a multitude of medical schools flourished within university systems, curricula were implemented with variable levels of success [1]. In the latter half of the 20th century, there remained an ongoing search for the ideal curriculum - as well as the methods themselves for curricular development.

A curriculum is defined as a sophisticated blend of educational strategies, course content, learning outcomes, educational experiences, assessment, the educational environment, and the individual students' learning style [3]. A process known as curriculum mapping has been described by Harden as a method to organize curricular contents. Curriculum mapping can help both educators and learners by displaying the key elements of a curriculum, and the relationships between them. Learners can identify what, when, where, how, and why they will learn, while educators can visualize their role within the entire curriculum. The scope and sequence of learning is made explicit, links with assessment are clarified, and curriculum planning becomes more effective and efficient. In this way the curriculum is more transparent to all of the stakeholders including the educators, the learners, the curriculum developer, and all other important stakeholders. The windows through which the curriculum map can be explored may include: (1) the expected learning outcomes; (2) curriculum content or areas of expertise covered; (3) assessment; (4) learning opportunities; (5) learning location; (6) learning resources; (7) timetable; (8) educators; (9) curriculum management; (10) learners. The key to a really effective, integrated curriculum is to get educators to exchange information about what is being taught and to coordinate this so that it reflects the overall goal. This can be achieved through curriculum mapping, which has become an essential tool for the implementation and development of a curriculum. Faced with curricula which are becoming more centralized and less departmentally based, and with curricula including both core and optional elements, the teacher may find that the curriculum map is the glue which holds the curriculum together [4].

All teaching activities, whether great or small can represent a curriculum. Beginning in the 1980's, Harden et al. [3] proposed a model for educational strategies in curriculum planning with the mnemonic SPICES:

$S \quad$ Student-centered, with students assuming responsibility for their own learning. This requires the student to decide their own learning objectives, decide the sequence and pace of learning, and assess their own progress, all under the guidance of a teacher.

P Problem-based, with application of knowledge to and derivation of knowledge from problems in clinical practice, health delivery, medical science and research.

I Integrated teaching, unifying subjects across academic subjects or departments such as anatomy, pathology, biochemistry, and clinical medicine.

C Community-oriented, with a goal of preparing students to ultimately work and serve in areas of health care need within the community

E Elective study periods, which incorporate some flexibility within the curriculum and give students the freedom to choose subjects and projects.

$\mathrm{S} \quad$ Systematic approaches, moving away from the apprenticeship model and emphasizing learning that is not "left to chance" but rather planned and recorded.

In 1998, David Kern composed a seminal work in the field of modern medical education, espousing a six-step approach to the formation and implementation of new curricula [5]. Since the initial publication of Kern's text, the means by which medical education are delivered have remained in as much flux as the state of American healthcare itself. With the implementation of the Affordable Care Act and its emphasis on improved access and quality of healthcare, new competencybased frameworks have been the scaffolding on which a multitude of new approaches to medical education have developed $[6,7]$. 


\section{Kern's framework for curriculum development}

Kern's foundational Framework for Curriculum Development [5] is comprised of six steps. While these steps are discussed in sequence below, it is important to keep in mind that the steps may occur in parallel as well as out of sequence; in fact, the various steps are intertwined and dependent on one another Figures 1-3.

1. Problem Identification and General Needs Assessment: Kern prompts educators to consider what prompts an educator to start work on a curriculum? Is the goal to impart new knowledge or a new technique? Or rather is it a reflection of demands from an accreditation agency or hospital administration? An important consideration when met with the idea (or demand) for a new curriculum is whether the resources required for design and implementation of a new curriculum is worth the time and effort involved - will it ultimately serve to improve a healthcare provider's knowledge base and therefore a health outcome? The essential component of this step is performing a general needs assessment, or problem identification. This is the gap between a current and desired approach to knowledge acquisition or how a health problem is addressed. There are many potential means to making these determinations including expert opinion, group consensus, or previously disseminated evidence. This step is important to justify later dissemination of the newly designed curriculum because it supports broader generalizability.

2. Targeted Needs Assessment: This is the step where the general needs assessment in Step 1 is applied to the actual learning environment and learners. Because a targeted needs assessment is the step where an ideal approach is superimposed upon the subject of interest, involvement of stakeholders (including teachers, learners, and administrators) is critical to appropriate framing of the problem. Herein is where one collects baseline information on the learners of interest. What are their knowledge base and known deficiencies? What motivates these learners? What are their preferred learning

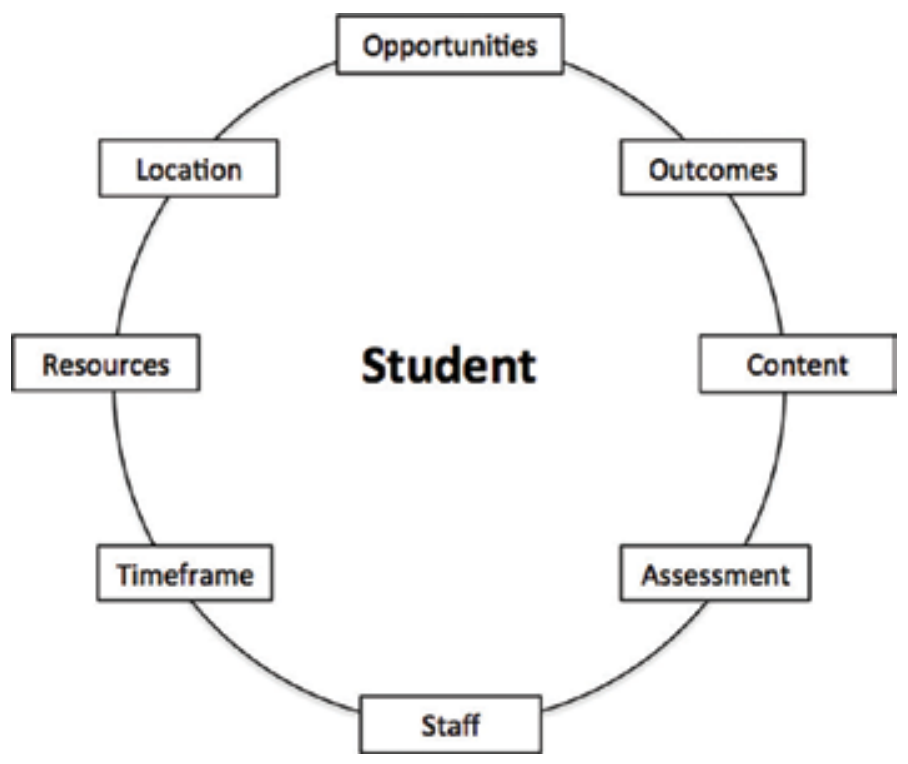

Figure 1.

Curriculum map (adapted from Harden [4]). 
styles? There are a multitude of assessment methods that can be employed (including informal discussion, interviews and focus groups, direct observation and exams). Ideally, when collecting this information, markers of accuracy and validity are included.

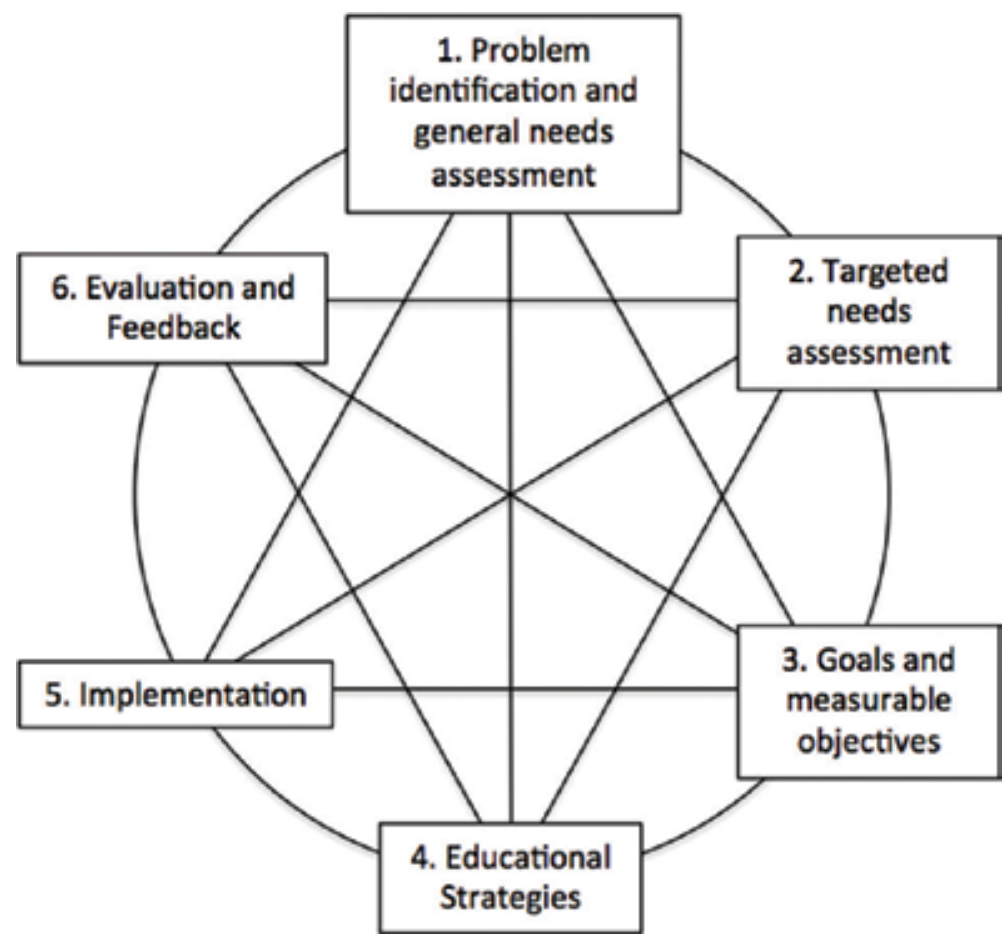

Figure 2.

Kern's curriculum development model (adapted from Kern [5]).

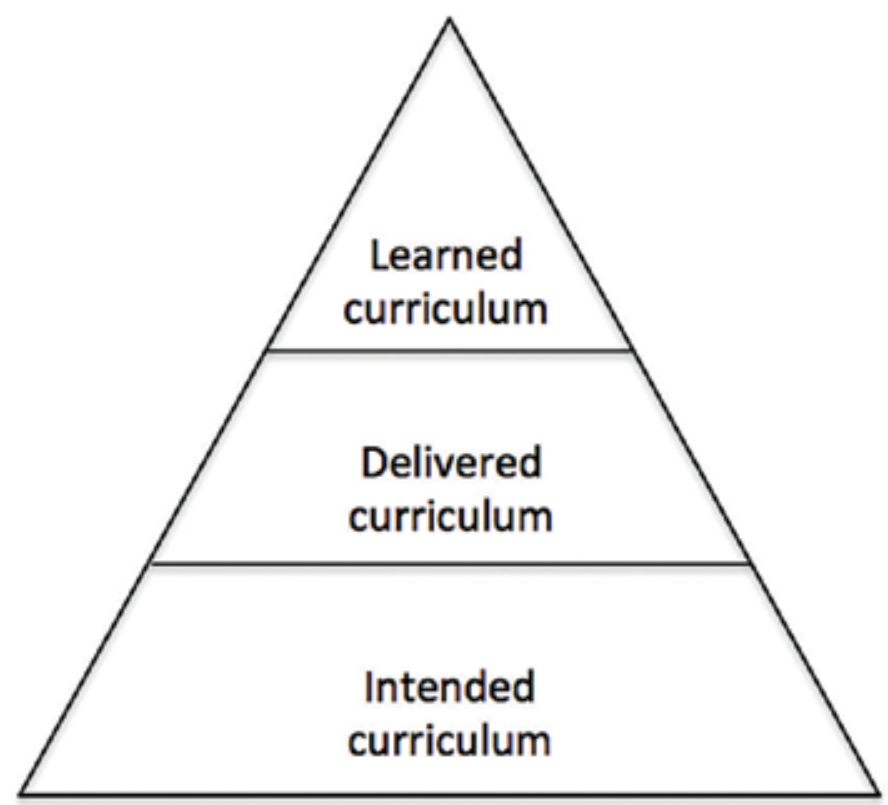

Figure 3.

Curriculum levels (adapted from Prideaux and Harden [4, 10]). 
3. Goals and Objectives: The development of strong curricular goals and objectives is vital to curriculum development. They define the expectations of the curriculum and guide the remainder of the developmental process. In considering this step, goals are defined as broad desires to accomplish while objectives are specific and measurable. An objective is the targeted result of a curriculum implementation and must be outlined prior to implementation to have a targeted result. Importantly, a goal must be well-written in order to be useful, and should follow this template: (who/will do/how much/of what/by when). Objectives can target the learners themselves, the educational process, or specific systems-level outcomes. Objectives should be developed using the SMART framework. This framework guides the educator in the development of objectives that are $\underline{\text { Specific, }}$ Measurable, $\underline{\text { Achievable, Realistic, and Time- }}$ based. Bloom's Taxonomy, the preeminent model for objective development, focuses on the use of action verbs describing the cognitive process by which learners work with knowledge. This approach is designed as a hierarchy where higher-level learning occurs as objectives are developed that are higher on the defined framework. Bloom's Taxonomy is often described as a pyramid, where the lowest level of learning objectives is at the bottom. In ascending order, these learning, action verbs include: remember, understand, apply, analyze, evaluate, and create [8]. When developing objectives, be sure to exercise caution. An overly exhaustive list of goals and objectives can overwhelm both educators and learners, limit creativity, and limit learner-centered education.

4. Educational Strategies: The job of a curriculum developer is to facilitate learning; however, there are a multitude of means by which this facilitation can occur. In the latter part of the 20th and in the 21st century, the process by which learners learn is increasingly better understood. The content of a curriculum naturally flows from learning objectives once they are established. Kern espouses using a variety of different strategies for reinforcement of a particular topic with the rationale that this will not only increase the degree of learner interest but also increase learner investment since they will inevitably realize their ideal learning methods reflected in the curriculum. Multiple educational methods should be used and chosen based on feasibility and available resources. For example, a simulation session should not be developed if the educators lack the necessary equipment. Similarly, educators should implement methods that are literature based, best methods for adult learners. Recently, self-directed learning has been increasingly recognized as an important aspect of medical education curricula due to its importance in continuing medical education. As a learning method, it should be incorporated into developed curricula due to the importance of the skill in lifelong learning. Not all learners are adept at this learning style; therefore, learners must be fostered with specific strategies for maximizing their self-directed learning practices.

5. Implementation: After the time, effort, and resources expended in the first four steps outlined above, the curriculum is finally ready for implementation. In order to successfully implement a curriculum, four stages will ideally occur: a) generating support from stakeholders including learners, instructors, and relevant administrators; b) plan for implementation of desired change including identification of resources such as personnel, time, facilities, and funding; c) operationalize the intervention, ideally planning for a pilot period, phase-in period, and full implementation of the curriculum; and d) ensure viability and longevity of the curriculum by involving multiple parties so the curriculum does not depend on the efforts of a single individual. 
6. Evaluation and Feedback: Ultimately, the contributions and accomplishments of the new curriculum should be recorded in a system of evaluation. Increased knowledge or ability can be marked by assessments on the level of the individual. Taken in aggregate, individual assessments can be used for evaluation of the program of instruction. What the evaluation encompasses is driven by the purpose of the evaluation itself - is it to judge the success of the program itself, and by what marker? Is it to justify the allocation of resources to further the mission of the curriculum? Is it to document the achievements of the individual who designed and implemented the curriculum? A successful curriculum is continually developing. In response to evaluative and feedback data, effective curricula remain dynamic and adjust based on the evolving needs of the learners. Finally, evaluations of a program once performed should be published for broader dissemination.

While Kern's framework is classically described as a six-step approach, an argument can be made regarding the inclusion of a seventh step, which involves dissemination of educational materials and outcomes. While Kern mentions this in his sixth step, its importance bears a separate step. The dissemination of educational materials has innumerable advantages. By disseminating educational materials, educators can increase collaboration, while receiving external feedback and peer review of educational materials. This ensures continued improvement of the educational product delivered. Similarly, physician educators lack time to complete all of the educational innovations they desire, By sharing educational materials, educators can minimize redundant work, especially since other educators are delivering similar content. Finally, by disseminating their educational materials and curricular outcomes, curriculum developers and educators can achieve recognition and academic advancement for their efforts.

\section{An evolving perspective on curriculum design}

The PRISMS model, proposed by Bligh [9] propagated new strategies for curriculum development including increased use of technology and problem-based learning, and emphasized the need for more clinical experience in medical school and more protected time for learning during residency. The PRISMS model can be broken down into each of its components.

- P: Product-focused, as the curriculum should emphasize applications to clinical practice and be practice-based. This includes acquisition of professional behaviors, application of knowledge to the real world, and feedback from patients.

- R: Relevance to communities and students, meaning that curricula should be planned around outcomes with a focus on local needs, and revised and reviewed frequently.

- I: Interprofessional, in that a culture of multiprofessional and interdisciplinary learning should be promoted, with emphasis of teamwork and collaboration between all persons involved in the care of the patient (eg. nursing, physical therapy, occupational therapy) with respect to clinical care, but also to education and research.

- S: Smaller class sizes and shorter courses, with units as building blocks implementing modern technology. 
- M: Multisite, further emphasizing the product-focused goal of the curricula allowing learners to ultimately care for patients in diverse settings including urban, rural, community sites, and academic teaching hospitals.

- S: Symbiotic, in that each of the above components combine to form a cohesive and coherent philosophy and product.

The guiding philosophy of the PRISMS model includes respect for autonomy of the adult learner while emphasizing group learning and reflection. Further, content must be context-based, relevant, and meaningful.

In 2003, Prideaux published an additional means to conceptualizing curriculum design [10]. In this work, a broader framework for curriculum design is described in which curricula fall under one of two models: prescriptive models, in which curriculum designers adhere to a stepwise guidebook to create curricula, and descriptive models, which describe what curriculum designers have accomplished once a curriculum has been implemented. The overarching goal for either design is that curriculum designers are agile in adapting to current environment, not continuing to teach that which is outdated. Additionally, curricula should develop in context of the community it was designed to serve in order to enhance health service provision.

Prideaux outlines the three sequential "levels" of a curriculum: 1) the planned curriculum, or what is intended 2) the delivered curriculum, or what is taught, and 3) the experienced curriculum, or what is actually learned by the intended audience. In this learner-centered concept, four main elements of a curriculum are identified: the content, strategies for teaching and learning, assessment of individuals, and evaluation of the program. Curriculum design is therefore organizing these four elements into a logical pattern of implementation. To communicate the implementation process to all parties, Prideaux promotes the use of a curriculum map [4], of which different versions can be created from the point of view of students, teachers, administrators, and accrediting authorities.

\subsection{Adult learning theory}

Facile adult educators continue to recognize that adults cannot be taught using traditional pedagogical approaches; in fact, adults are always voluntary learners that can disappear from learning experiences that do not satisfy or engage them. The practice of educating adults has been deviating from traditional pedagogical approaches for some time; therefore, the technology of andragogy was developed. Andragogy refers to the science of adult education. Important conditions of learning and associated principles of teaching developed by Malcolm Knowles that define andragogy are illustrated in Table 1.

Curricula and associated educational materials should be developed using literature-based best practices in adult learning theory. Andragogy, the science of adult learning, differs substantially from traditional pedagogical approaches. Adult learning is most clearly described using seven core principles. Adult learning builds from established learning needs, is practical and problem-centered, necessitates a positive learning climate, occurs when new ideas are integrated with existing knowledge, promoted when respected by both educators and other learners, is self-directed, and builds on previous experience [11]. Ultimately, curricula should employ educational materials that create a climate of assisting learners to acquire information and incorporating their experiences to master the content through session structure. Finally, curricula should help learners test their ideas, retain new information, and apply that information clinically [11]. Adult learning is most successful when learning is active and problem-centered, learners take ownership of the classroom and are accountable for demonstrating mastery, and feel safe, respected and successful [12]. 


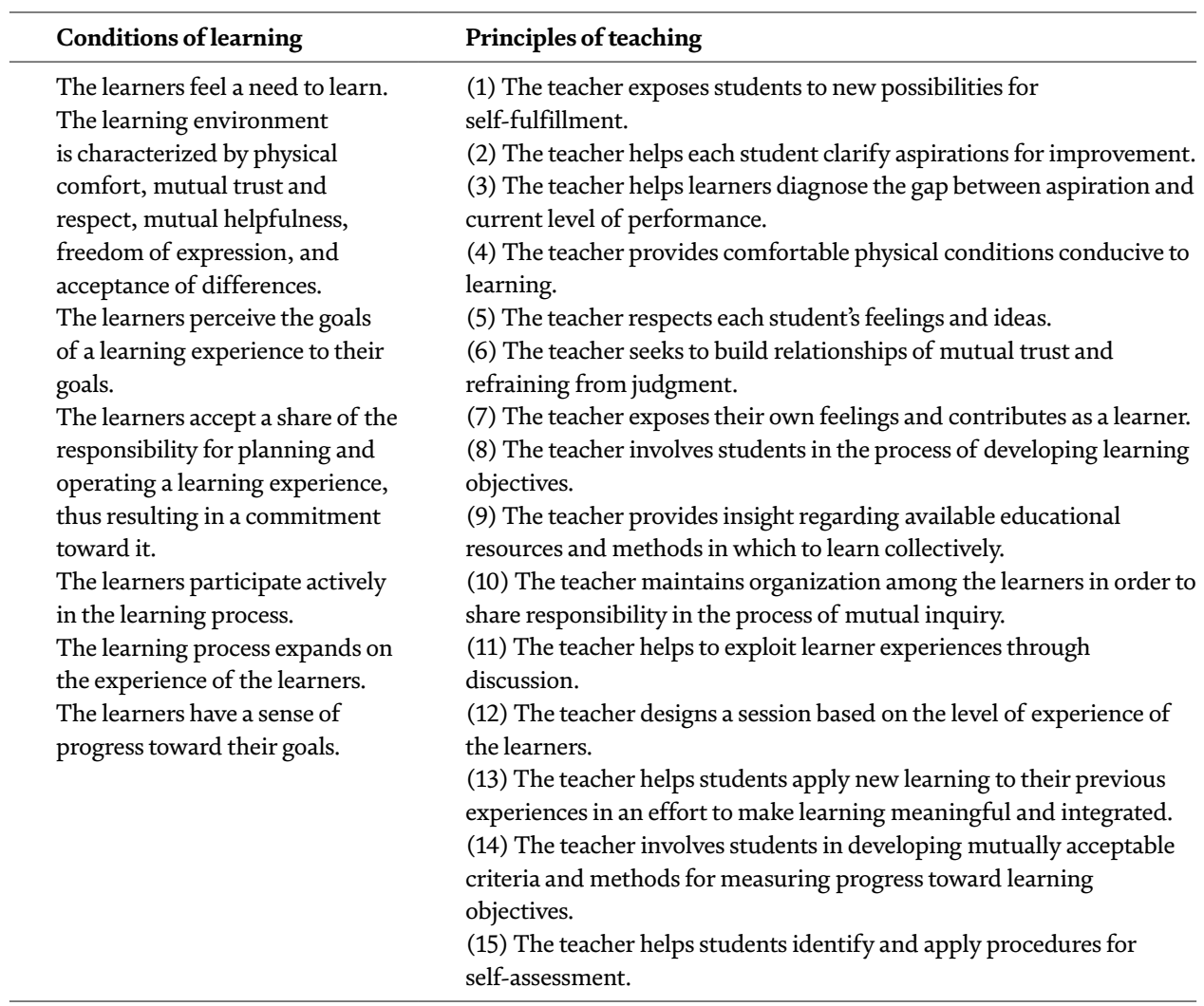

Table 1.

Andragogical conditions of learning and principles of teaching (adapted from Knowles [11]).

\subsection{Flipped classroom pedagogy}

Medical education continues to evolve by shifting from traditional, lecture based teaching to other educational approaches that promote higher-order learning and active learner participation [13]. The flipped classroom method, one particular learning model, has become increasingly popular in medical education. In this educational pedagogy, learning materials are consumed independently in a learner-paced manner at home, while classroom time is devoted to knowledge application, case-based learning, or active discussion and problem solving. Conversely, within a traditional classroom, foundational knowledge is often passively transferred to learners through lectures delivered by instructors. Following the teaching session, learners actively use and apply the knowledge outside of the educational setting $[14,15]$.

Influenced by the preference of millennial learners for immediate, digital educational materials, medical educators are constantly searching for the most efficient and effective approach [16]. Active, self-directed learning, a necessary component of this learning model, is a vital skill that provides the foundation for adult learning and continuing education [15]. Additionally, this model is also supported by the educational theory of social constructivism $[17,18]$. Group collaboration encourages modeling, scaffolding, and feedback that engage learner preconceptions and build upon existing understanding [19]. This model promotes a higher level of learning, defined by Bloom as analysis, synthesis, and evaluation $[8,19,20]$. As a result, increasing numbers of educators have adopted the flipped classroom strategy in both medical and more traditional educational settings [21-28]. 


\section{Contemporary applications of curriculum design}

\subsection{Simulation in medical education}

One unique application of curriculum design is in the field of simulation in healthcare. Simulation has long been used in fields outside of medicine including aviation and commercial airlines, aerospace, and the military. Simulation in healthcare goes as far back as the 18th century when models of the pelvis were used by midwives for newborn delivery instruction. In the modern era, simulation in healthcare, initially pioneered by anesthesia, is now broadly adopted by many specialties including emergency medicine, critical care, trauma, obstetrics, pediatrics, and radiology [20]. Simulation is employed as early as the pre-clinical years in medical school, where applications include clinical skills, clinical medicine, physical diagnosis, anatomy and physiology. In the second half of medical school during clinical clerkships, the specialties which most commonly employ simulation are internal medicine, pediatrics, and emergency medicine. In residency, nearly $90 \%$ of programs report some use of simulation, most frequently in the fields of internal medicine, emergency medicine, general surgery, pediatrics, anesthesia, and obstetrics and gynecology [30]. This broad use of simulation has been ushered in by the modern era of healthcare delivery in which there is an expectation that practitioners are prepared to care for patients prior to encountering a pathology or procedure, with less reliance on serendipity to acquire the wide range of skills necessary for clinical practice but instead methodically structuring a curriculum within an outcomes-based framework with assessments and monitoring at regular intervals [31].

Simulation is broadly defined as "advanced technologies recreating the clinical experience" [31], with the goal to train healthcare practitioners to safely conduct patient care. More specifically, it is the imitation of the operation of a real-world process or system over time, with the ability to show real effects of conditions and actions, or when a real system cannot be engaged because it is either not accessible, dangerous, or does not exist [29]. Simulation encompasses cognitive and affective domains and encompasses a wide range of techniques and approaches.

The types of simulation can be framed by the degree of fidelity and the means by which the simulation curriculum is delivered. The degree of fidelity, or extent to which the system mimics real life, in simulation can be gauged by how similar the simulation model is to real life with respect to equipment, environment, and physiological parameters [31]. There are many vehicles for delivery of simulationbased learning, including partial task trainers (ie a model arm for venipuncture), computer-based models, virtual reality and haptic systems (with kinaesthetic and tactile sensation such as for robotic surgery), integrated simulators which employ manikins and computer-controlled outputs such as vital signs, simulated or standardized patients, and simulated environments such as for disaster training.

Steps in the curriculum design in simulation include acquisition of valid source information, use of simplifying approximations and assumptions, and evaluation of the fidelity and validity of simulation outcomes [32].

McGaghie [33] lists features and best practices within simulation curriculum design and implementation, which include:

\section{1. feedback}

2. deliberate practice

3. curriculum integration 
4. outcome measurement

5. simulation fidelity

6. skill acquisition and maintenance

7. mastery learning

8. transfer to practice

9. team training

10. high stakes testing

11. instructor training

12. educational and professional context

These features and best practices can be applied to the principles of curriculum development laid out in Kern's work, such as in developing a simulation-based mastery learning curriculum [34].

\subsection{Massive open online courses}

Although Kern's framework for curriculum development remains foundational to medical education, new approaches and applications to this framework continue to shape its application in the 21st century. One novel approach in the context of collaborative online learning models such as Massive Open Online Courses (MOOC), which are hailed as a paradigm for graduate medical education [13] . A course meets the definition of a MOOC when a single course has thousands enrolled (massive), and can share information through meaningful and free (open) online learning environments (online courses) [35, 36]. MOOC were originally based on curricula models in higher education but are distinct from campus courses in that they typically have discussion fora where recommended readings and short videos are discussed in online fora by diverse learners [36]. Further, assessments are all carried out online. The benefit of MOOC are that they can involve learners with a more diverse range of backgrounds, enriching courses beyond education that can be delivered by the host institution alone [37]. Based on the concept of MOOC, online courses have proliferated. MOOC have the ability to expand on known means of computer-based learning in medical education.

A publication by Goldberg and colleagues [35] addresses the availability of MOOC on medical topics. They are advocates for MOOC as an innovative medical education tool for many reasons. With the foundational philosophy that quality health care needs to be effective, high-quality, equitable, patient and family-centered, and delivered by an interprofessional team comprised of educators from all professional programs, the MOOC may have a unique advantage in medical education. MOOC have the inherent potential of educating consumers, students, and healthcare providers alike; the MOOC format has no constraints of time, geography, or level of education, and offers the advantage of being asynchronous. From a big picture standpoint, it has the potential to strengthen communication and foster collaboration nationally and internationally while increasing public health literacy for patients, model innovation for learners, and offer continuing medical education for providers. Although detractors may assert that MOOCs will undermine 
traditional methods of education, a more cohesive view would espouse that while MOOC cannot fully supplant existing curricula, they can be complementary and augment existing forms of medical education. Goldberg et al. reviewed existing offerings for MOOC on medical topics, finding that the majority of topics address education for chronic care conditions, health literacy for the general public, and evidence-based medicine for healthcare providers. Further research ranking the relevance of MOOC to post-graduate training reveals that many courses are relevant and applicable, course duration and workloads appropriate for physicians [38].

As the role of MOOC in medical education is emerging and evolving, further literature has since been published which offers a prescriptive view of how to implement a MOOC [37]. Additional acknowledged roles for MOOC in medical education include integration within campus courses, increasing interprofessional collaboration, enabling the flipped classroom, and for continuing medical education. Within these areas, Pickering and colleagues introduce a framework for developing, delivering, and evaluating a MOOC, with the following stepwise advice:

1. Enroll in a MOOC to experience the format first-hand

2. Learn from other MOOC enthusiasts to understand practical implementation and time commitment; network for multi-institution MOOC or between departments

3. Develop a MOOC topic that you are passionate about, accounting for intended audience and MOOC length; perform a preliminary search of what is available within this topic. Pitch to intended audience to gauge enthusiasm.

4. Recruit a committed and enthusiastic team to support the MOOC's development and delivery, including a lead academician to develop the curriculum, write lecture scripts, create learning objectives, and set assessment questions. Recruit colleagues to review the curriculum, and create faculty development programs addressing how to support learners on a MOOC.

5. Develop a curriculum map to guide content development, keeping in mind the target audience, academic level of content, and duration of the course. The map should be accessible to learners to serve as a scaffold on which they can track their own progress.

6. Create a clear project plan to deliver the MOOC. The authors estimate that it takes approximately one year to create the content for a single course.

7. Create video content based on good educational practice - short, with a conversational style, and tailored to the course.

8. Construct an assessment profile, keeping in mind scalability to thousands of learners who require timely and accurate feedback. The format which lends itself best to these requirements is multiple choice quizzes, but other options include peer assessments.

9. Promote your course with a clear message, aspiring to both a local and global audience.

10. Provide a supportive learning environment with which your learners can interact, including guidance to the learner on how to use the MOOC. 
11. Devise a strategy to evaluate the course after implementation, which may include course enrollment and completion data, pre- and post-test results, and targeted questionnaires for a subset of learners.

12. Share the experience of creating a MOOC through discussion and publication to aid colleagues with a similar interest.

\subsection{Free open access medical education}

Free Open Access Medical Education (FOAM or FOAMed) is defined as "a collection of interactive online medical education resources" including blogs, podcasts, tweets, videos, and other web-based media. It is "educational social media for medicine" with open sharing and collaboration with attribution and recognition of the work of others [39]. The object of FOAM is to form an online community to share ideas and accelerate translation of research into clinical practice [40].

FOAM is a sphere of medical education which has expanded rapidly in recent years, led by the specialties of Emergency Medicine and Critical Care [40]. In one study examining expansion of FOAM, in the time period from 2002 to 2013 the number of FOAM resources available grew from two blogs and one podcast to 141 blogs and 42 podcasts [41]. By the most recent estimate this number has expanded to 240 [39]. While the United States, Australia, and the United Kingdom lead in countries who predominantly consume FOAM, the potential for dissemination of medical knowledge via FOAM in lower resource countries has also been studied [40]. Outside of Emergency Medicine and Critical Care, the use of FOAM has been detailed in Emergency Medical Services [42, 43] and Pediatrics [44].

Since the inception of FOAM, some guidelines have been published from expert users on the optimal means to using FOAM and how to evaluate the quality of resources. Weingart has established a beginner's guide to FOAM with a stepwise hierarchy in which learners can progress from novice to expert: existence (create online profile), safety (learn the rules of engagement to avoid problems), consumption (learn to effectively use resources), collaboration (engage with others), creation (of content) [45]. In another approach, a collection of four strategies to evaluate and engage in FOAM are 1. cultivate digital mentors 2. browse the most popular FOAM websites 3. use critical appraisal tools for FOAM and 4. contribute new online content.

A multitude of applications of FOAM have been promoted, including integration into existing graduate medical education curricula. General guidelines and suggestions for use have been proposed, including promotion of approved FOAM websites by a residency program to residents, creation of a residency Twitter account to interact with users, involving residents and faculty in creating and sharing FOAM, and application in flipped classroom and asynchronous curriculum models $[46,47]$. In one training program, application of FOAM in the curricula has been accomplished via a model of team-based learning using the Academic Life in Emergency Medicine (ALiEM) Approved Instructional Resources (AIR) series, allowing faculty oversight and discussion of clinical applications [48]. The format of FOAM naturally adapts to the asynchronous component of residency education and courses such as "Asynchrony" incorporate an assignment, discussion, and quiz into the curriculum [49]. Additional attention has been paid to the use of podcasts in medical education. In comparing media, learners have been shown to improve their knowledge base equally with blogs and podcasts [50].

Remarkably, in a survey of trainees in Emergency Medicine, the podcast was found to the most popular means of obtaining medical knowledge, with reported 
use by $35 \%$ of residents surveyed compared to $33.6 \%$ of residents who report predominantly reading textbooks. Podcasts were additionally endorsed as the most beneficial means of learning [51]. The use of podcasts by learners has also been detailed in undergraduate medical education in a study examining usage conditions (most popular during driving, chores, and exercising) and knowledge retention (increased compared to the existing undergraduate curriculum alone) [52].

In addition to podcasts, Twitter has emerged as another medium for exchange of information in medical education [47]. The concept of an online community of practice exists wherein an open digital space and non-hierarchical structure promote information sharing, and knowledge translation. Further, social media-based platforms such as Twitter allow for the development of a strong group identity among educators and learners who partake.

The importance of critical appraisal and vetting educational content of FOAM cannot be emphasized enough. Due to the nature of FOAM, in which anyone can be a consumer or producer of online content, not all resources are of equal quality. Many FOAM articles and resources can be opinion-based and not all equally grounded in evidence-based medicine. Further, it can be hard for the user to discern whether content draws predominantly from the former or the latter. Multiple index markers have been developed to determine the quality of resources from which a learner is gaining information. These include adherence to the Health On the Net code of conduct, and application of the DISCERN score, which were developed to appraise online resources for patient use. With respect to medical education for practitioners, metrics to determine the quality of online resources such as the Quality Checklists for Blogs and Podcasts, ALiEM AIR score, and METRIQ score [53] have been newly developed and applied.

Despite the propagation and application of these quality markers, detractors of FOAM do list downsides of the format including little editorial oversight of material, overly rapid translation of information to patient care, and the outsize influence of figures with an eminent online presence [54]. When queried, the FOAM resources recommended by trainees and attending physicians varies widely and is also not a reliable indicator of quality [55].

With respect to representation of core content for learners in FOAM, in one study of core content for Emergency Medicine trainees, FOAM related to airway management, interpretation of EKGs, propagation of new research and evidencebased medicine, resuscitation, and point-of-care ultrasound were overly represented compared to the least represented topics of cutaneous disorders, hematologic disorders, atraumatic musculoskeletal disorders, and obstetrics and gynecology [56]. Despite FOAM's popularity in recent years, FOAM alone is not sufficient for trainees to learn all areas relevant to practice in a specialty.

With respect to FOAM and MOOC as they pertain to curriculum developers, a novel approach to curriculum development implemented by Shappell et al. [57] involves crowdsourcing each step of curriculum development as it pertains to the Emergency Medicine residency curriculum. In Shappell et al.s scoping review, an expert panel of authors searched for key terms within each step of curriculum development with inclusion of references to FOAMed. In determining the extent to which each of Kern's six steps are referenced in FOAMed, they were able to gain an understanding that the steps of curriculum development which are underrepresented, which include (1) articulating goals and objectives and (2) tools for curricular evaluation. Ultimately, it is the authors' goal and assertion that crowdsourcing curriculum development will diffuse the burden of creating a comprehensive online learning center and will help developers consider how their contributions will align with the work of others.

One example of a successfully-implemented FOAMed curriculum is the Foundations of Emergency Medicine curriculum for emergency medicine 
residents [58]. Initially developed for emergency medicine interns at Northwestern University in 2014, the Foundations curriculum uses a flipped-classroom model to provide a longitudinal year-long course for understanding cardinal presentations and management strategies for "cannot miss" diagnoses. Since its inception at a single institution, the Foundations curriculum has rapidly expanded across more than a hundred institutions to encompass all levels of resident learners, accomplished through crowdsourcing from educational leaders across the field. Much of its expansion and success can be attributed to its FOAMed format, as the curriculum is readily accessible to all residency leadership in emergency medicine.

\section{Conclusion}

The concept of curriculum development has come a long way since the inception of medical training in the United States and the formalization of medical education. As the Flexner Report ushered in a new era shifting focus from reliance on lectures and textbooks to the real-life application of medical knowledge, medical education was further shaped by the implementation of post-graduate training. In parallel, the concept of curriculum development for educators of both medical students and resident trainees took hold. Strategies for educators such as Harden's curriculum mapping and Kern's landmark six-step approach for curriculum design proved seminal to the field, while new understanding in adult learning theory shaped the means by which information was propagated. In the contemporary era, new advances in technology have allowed for radical models for medical education to spring forth, including the use of simulation, massive open online courses, and free open access medical education. Based on the advances of the past few decades, the future of curriculum design in medical education is hard to predict but is sure to hold even more innovation.

\section{Conflict of interest}

The authors declare no conflicts of interest.

\section{Thanks}

Dr. Li-Sauerwine wishes to thank her family for their support during the writing of this chapter.

Dr. King wishes to thank his family for their support in all professional endeavors. He also wishes to thank Dr. Sorabh Khandelwal for introducing him to curriculum development, and providing tireless mentorship and professional development. 


\section{Author details}

Simiao Li-Sauerwine* and Andrew King

Department of Emergency Medicine, The Ohio State University,

Columbus, Ohio, USA

*Address all correspondence to: simiao.li-sauerwine@osumc.edu

\section{IntechOpen}

(C) 2018 The Author(s). Licensee IntechOpen. This chapter is distributed under the terms of the Creative Commons Attribution License (http://creativecommons.org/licenses/ by/3.0), which permits unrestricted use, distribution, and reproduction in any medium, provided the original work is properly cited. (cc) BY 


\section{References}

[1] Ludmerer KM. Time to Heal: American Medical Education from the Turn of the Century to the Era of Managed Care. Oxford: Oxford University Press; 1999

[2] Flexner A, Updike DB, Carnegie Foundation for the Advancement of Teaching., \& Merrymount Press. Medical Education in the United States and Canada: A Report to the Carnegie Foundation for the Advancement of Teaching. 576 Fifth Avenue. New York City: publisher not identified; 1910

[3] Harden R, Sowden S, Dunn WR. Educational strategies in curriculum development: The SPICES model. Medical Education. 1984;18(4):284-297

[4] Harden R. Curriculum mapping: A tool for transparent and authentic teaching and learning. Medical Teacher. 2000;23(2):123-127

[5] Kern DE. Curriculum Development for Medical Education: A Six Step Approach. Baltimore: Johns Hopkins University Press; 1998

[6] Cooke M, Irby DM, O’Brien BC, Shulman LS. Educating Physicians: A Call for Reform of Medical School and Residency. San Francisco: Jossey-Bass; 2010

[7] Harden R, Crosby J, Davis M. Outcome based education: Part 1-An introduction to outcomesbased education. Medical Teacher. 1999;21(1):7-14

[8] Bloom BS, Engelhart MD, Furst EJ, et al. The classification of educational goals. In: Bloom BS, editor. Taxonomy of Educational Objectives. Handbook I: Cognitive Domain. David McKay: New York, NY; 1956

[9] Bligh J, Prideaux D, Parsell G. PRISMS: New educational strategies for medical education. Medical Education. 2001;35:520-521

[10] Prideaux D. ABC of learning and teaching in medicine: Curriculum design. BMJ. 2003;326(7383):268-270

[11] Knowles MS. The Modern Practice of Adult Education: From Pedagogy to Andragogy. Wilton, Conn: Association Press; 1980

[12] Carey B. How We Learn: The Surprising Truth about when, where, and why it Happens. 1st ed. New York: Random House; 2014. p. 254. ISBN: 978-0-8129-8429-3

[13] Mehta NB, Hull AL, Young JB, et al. Just imagine: New paradigms for medical education. Academic Medicine. 2013;88:1418-1423

[14] Chen F, Lui AM, Martinelli SM. A systematic review of the effectiveness of flipped classrooms in medical education. Medical Education. 2017 Jun;51(6):585-597

[15] King A, Boysen-Osborn M, Cooney R, et al. Curated collection for educators: Five key papers about the flipped classroom methodology. Cureus. 2017;9(10):e1801

[16] Cooper AZ, Hsieh G, Kiss JE, et al. Flipping out: Does the flipped classroom learning model work for GME? Journal of Graduate Medical Education. 2017 Jun;9(3):392-393

[17] Vygotsky LS. Mind in Society: The Development of Higher Psychological Processes. Cambridge, MA: Harvard University Press; 1978

[18] Haidet P, Morgan RO, O’Malley $\mathrm{K}$, et al. A controlled trial of active versus passive learning strategies in a large group setting. Advances in Health Sciences Education: Theory and Practice. 2004;9(1):15-27 
[19] Riddell J, Jhun P, Fung C, et al. Does the flipped classroom improve learning in graduate medical education? Journal of Graduate Medical Education. 2017 Aug;9(4):491-496

[20] Sherbino J, Chan T, Schiff K. The reverse classroom: Lectures on your own and homework with faculty. CJEM. 2013 May;15(3):178-180

[21] Prober CG, Khan S. Medical education reimagined: A call to action. Academic Medicine. 2013;88:1407-1410

[22] McLaughlin JE, Roth MT, Glatt DM, et al. The flipped classroom: A course redesign to foster learning and engagement in a health professions school. Academic Medicine.

2014;89(2):236-243

[23] Leung JY, Kumta SM, Jin Y, et al. Short review of the flipped classroom approach. Medical Education.

2014;48(11):1127

[24] Nematollahi S, St John PA, AdamasRappaport WJ. Lessons learned with a flipped classroom. Medical Education. 2015;49(11):1143

[25] Morgan H, McLean K, Chapman C, et al. The flipped classroom for medical students. The Clinical Teacher. 2015 Jun;12(3):155-160

[26] Vincent DS. Out of the wilderness: Flipping the classroom to advance scholarship in an internal medicine residency program. Hawaii Journal of Medicine \& Public Health. 2014;73 (11 suppl 2):2-3

[27] Ramar K, Hale CW, Dankbar EC. Innovative model of delivering quality improvement education for trainees-A pilot project. Medical Education Online. 2015;20:28764

[28] Sadosty AT, Goyal DG, Hern HG Jr, et al. Alternatives to the conference status quo: Summary recommendations from the 2008 CORD academic assembly conference alternatives workgroup. Academic Emergency Medicine. 2009;16(suppl 2):25-31

[29] Issenberg SB, Gordon MS, Dl G, Safford RE, Hart IR. Simulation and new learning technologies. Medical Teacher. 2001;16:16-23

[30] Passiment M, Sacks H, Huang G. Medical simulation in medical education: Results of an AAMC survey. Washington, D.C; In: Association of American Medical Colleges. 2011

[31] Ker J, Bradley P. Simulation in medical education. In: Swanwick $\mathrm{T}$, editor. Understanding Medical Education: Evidence, Theory, and Practice. London, UK: Wiley-Blackwell; 2010. pp. $164-180$

[32] Shah C, Kumar V, Knoche C. Simulation in medical education. International Journal of Basic and Applied Physiology. 2012;1(1):167-170

[33] McGaghie WC, Issenberg SB, Petrusa ER, Scalese RJ. A critical review of simulation-based medical education research: 2003-2009. Medical Education. 2010;44:50-63

[34] Barsuk JH, Cohen ER, Wayne DB, Siddall VJ, McGaghie WC. Developing a simulation-based mastery learning curriculum: Lessons from 11 years of advanced cardiac life support. Simulation in Healthcare. 2016 Feb;11(1):52-59

[35] Goldberg LR, Crocombe LA. Advances in medical education and practice: Role of massive open online courses. Advances in Medical Education and Practice. 2017 Aug 21;8:603-609

[36] Hoy MB. MOOCs 101: An introduction to massive open online courses. Medical Reference Services Quarterly. 2014;33(1):85-91 
[37] Pickering JD, Henningsohn L, DeRuiter MC, de Jong PGM. Reinders MEJ5. Twelve tips for developing and delivering a massive open online course in medical education. Medical Teacher. 2017 Jul;39(7):691-696

[38] Subhi Y, Andresen K, Rolskov Bojsen S, Mørkeberg Nilsson P, Konge L. Massive open online courses are relevant for postgraduate medical training. Danish Medical Journal. 2014 Oct;61(10):A4923

[39] Nickson CP, Cadogan MD. Free open access medical education (FOAM) for the emergency physician. Emergency Medicine Australasia. 2014 Feb;26(1):76-83

[40] Burkholder TW, Bellows JW, King RA. Free open access medical education (FOAM) in emergency medicine: The global distribution of users in 2016. The Western Journal of Emergency Medicine. 2018 May;19(3):600-605

[41] Cadogan M, Thoma B, Chan TM, Lin M. Free open access Meducation (FOAM): The rise of emergency medicine and critical care blogs and podcasts (2002-2013). Emergency Medicine Journal. 2014 Oct;31(e1):e76-e77

[42] Bucher J, Donovan C, McCoy J. EMS providers do not use FOAM for education. International Journal of Emergency Medicine. 2018 May 24;11(1):27

[43] Mason P, Batt AM. \#FOAMems: Engaging paramedics with free, online open-access education. Journal of Education Health Promotion. 2018 Mar 1;7:32. DOI: 10.4103/jehp.jehp_84_17 eCollection 2018

[44] Baker M, Long N, Parker C. The world of FOAM: A practical guide to free online paediatric education resources. Journal of Paediatrics and Child Health. 2016 Feb;52(2):105-108
[45] Weingart SD, Thoma B. The online hierarchy of needs: A beginner's guide to medical social media and FOAM. Emergency Medicine Australasia. 2015 Feb;27(1):5

[46] Otterness K. Incorporating FOAM into medical student and resident education. Clinical and Experimental Emergency Medicine. 2017 Jun 30;4(2):119-120. DOI: 10.15441/ ceem.16.196 eCollection 2017 Jun

[47] Roland D, Spurr J, Cabrera D. Preliminary evidence for the emergence of a health care online Community of Practice: Using a Netnographic framework for twitter hashtag analytics. Journal of Medical Internet Research. 2017 Jul 14;19(7):e252

[48] Fallon T, Strout TD. Free open access medical education (FOAM) resources in a team-based learning educational series. The Western Journal of Emergency Medicine. 2018 Jan;19(1):142-144

[49] Pensa G, Smith J, McAteer

K. Calling all curators: A novel approach to individualized interactive instruction. The Western Journal of Emergency Medicine. 2018 Jan;19(1):169-171

[50] Lien K, Chin A, Helman A, Chan TM. A randomized comparative trial of the knowledge retention and usage conditions in undergraduate medical students using podcasts and blog posts. Cureus. 2018 Jan 15;10(1):e2065

[51] Mallin M, Schlein S, Doctor S, Stroud S, Dawson M, Fix M. A survey of the current utilization of asynchronous education among emergency medicine residents in the United States. Academic Medicine. 2014 Apr;89(4):598-601

[52] Chin A, Helman A, Chan TM. Podcast use in undergraduate medical education. Cureus. 2017 Dec 9;9(12):e1930 
[53] Lo A, Shappell E, Rosenberg H, Thoma B, Ahn J, Trueger NS, et al. Four strategies to find, evaluate, and engage with online resources in emergency medicine. CJEM. 2018 Mar;20(2):293-299

[54] Chan T, Trueger NS, Roland D, Thoma B. Evidence-based medicine in the era of social media: Scholarly engagement through participation and online interaction. CJEM. 2018 Jan;20(1):3-8

[55] Krishnan K, Thoma B, Trueger NS, Lin M, Chan TM. Gestalt assessment of online educational resources may not be sufficiently reliable and consistent. Perspectives on Medical Education. 2017 Apr;6(2):91-98

[56] Stuntz R, Clontz R. An evaluation of emergency medicine Core content covered by free open access medical education resources. Annals of Emergency Medicine. 2016 May;67(5):649-653.e2

[57] Shappell E, Chan TM, Thoma $\mathrm{B}$, et al. Crowdsourced curriculum development for online medical education. Cureus. December 08, 2017;9(12):e1925

[58] Moore KG. Foundations in Action. 2018 September 6. Retrieved from: https://foundationsem.com/ foundations-in-action/ 



\title{
Chapter 4
}

\section{Resident Autonomy}

\author{
Amanda Cooper and Steven Allen
}

\begin{abstract}
Autonomy in medical training is required to develop independent and competent physicians. The way in which this incremental level of independence is granted to a trainee must be thoughtful and deliberate to ensure appropriate supervision and patient safety. Theories that support the role of autonomy will be introduced and discussed in this chapter. Ethical considerations that describe the implications of balancing the necessary independence for trainees and an attending physician's responsibility to the patient and the patient's safety will also be considered. The level of autonomy that is granted is the responsibility of both the attending physician and trainee so that it is not only appropriate but also wellearned. There are multiple tools that may be used to objectively measure one's competence and necessary level of autonomy based on performance that will be discussed within this chapter. Finally we will demonstrate that encouraging and striking the balance of supervision and autonomy may be done safely with appropriate patient outcomes and trainee development into independent physicians. These outcomes will help to encourage autonomy amongst medical trainees, no matter one's specialty, to train and develop competent, independent physicians of the future.
\end{abstract}

Keywords: autonomy, self-determination theory, entrustment, entrustable professional activities, graduate medical education, Zwisch scale, graduated responsibility

\section{Introduction}

Successfully training residents to become competent, independent physicians requires balancing supervision and autonomy. Autonomy in residency has been previously defined as the ability of a resident "to manage patients on his or her own" [1]. Residents, teaching faculty, hospital administrators, and members of the general public all understand the importance of autonomy in training for developing the skills necessary for independent practice [1,2]. The Accreditation Council for Graduate Medical Education (ACGME), the organization that oversees residency programs in the United States, specifies that "the privilege of progressive authority and responsibility, conditional independence, and a supervisory role in patient care delegated to each resident must be assigned by the program director and faculty members" [3]. Benefits of autonomy include increased resident self-confidence, engagement in learning, sense of ownership of patients, and self-directed learning $[1,2]$. The benefits of autonomy, however, must be balanced (Figure 1) against the potential risk to patient safety. In the years since the 1999 publication of To Err is Human, the Institute of Medicine's report on patient safety [4], the focus of the healthcare field on safety has increased, resulting in more 


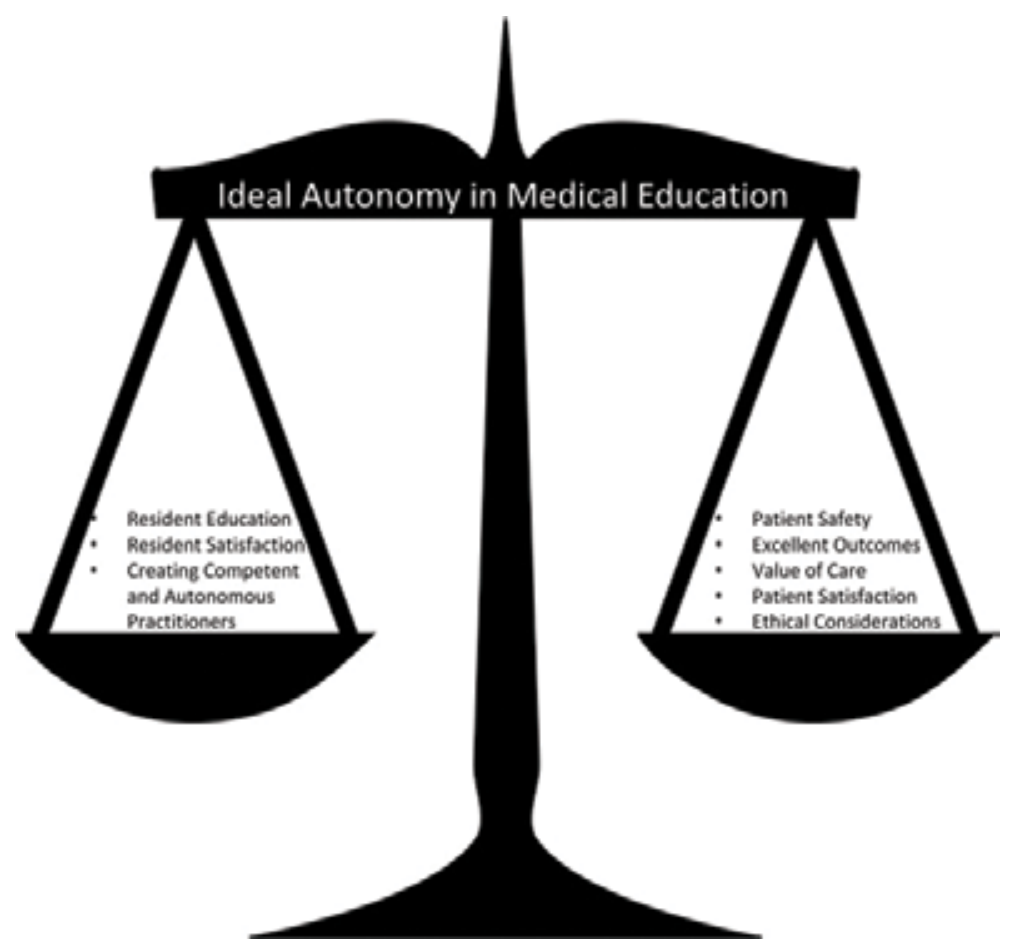

Figure 1.

Balance of resident autonomy and patient outcomes.

stringent requirements for supervision of residents [3], which has contributed to a trend in decreased autonomy for residents and may be resulting in recently graduated trainees who are entering the workforce less prepared than their predecessors were. Concerns about these trends have led to a recent focus on resident autonomy within the medical education community.

In this chapter we will discuss the theories that support the incredibly important subject of autonomy in medical education. Furthermore, we will review the ethical issues surrounding autonomy amongst trainees and the attending physicians' obligation to providing quality and safe medical care to their patients. Additionally, we will describe methods of granting autonomy and objective measures to determine how much autonomy should be granted. Finally we will discuss the outcomes associated with increased autonomy amongst trainees in medicine and finally describe methods that help to promote autonomy all while keeping patients safe.

\section{Theories supporting autonomy}

\subsection{Self-determination theory}

Self-determination theory is a validated motivational theory that has been often applied to education. Self-determination theory states that autonomy, competence, and relatedness are powerful motivators of intrinsic motivation and are important factors in well-being [5]. Intrinsic motivation is critical for learning. Self-determination theory provides a powerful argument in favor of not only maintaining, but working to increase autonomy in medical trainees. As Ten Cate, Kursurkar, and Williams have argued, "High IM [intrinsic motivation], e.g., learning out of interest, curiosity or enjoyment, and autonomous forms of self-regulation 
are associated with better learning, better conceptual understanding, better academic performance and achievement and higher levels of well-being than high extrinsic motivation" [6]. These outcomes are ones that all medical educators aspire for their learners to achieve.

An important caveat about autonomy, however, is that "autonomy does not mean acting without help from others, it means having feelings of volition and free will in whatever actions are carried out" [6]. This underscores the role the educator can play in supporting autonomous decision making in trainees. In particular, for those learners who may be more novice or struggling with obtaining competence, the supervising physician can provide scaffolding [7] (discussed further below) and other types of autonomy support, which includes asking the learner for personal goals, encouraging questions, answering questions in a thoughtful way, and avoiding judgment about prior behavior [6].

\subsection{Expectancy theory}

Work-motivation theories try to account for individuals' choice of tasks to pursue and their performance on and persistence in those tasks [8]. Expectancy theory is one such theory, which postulates that expectancy, one's belief about how well one will do on future tasks (vs. ability, which is one's belief about one's current competence) [9], is one of the main factors explaining the amount of time and effort one is willing to put into an activity. Expectancy theory also states "that a person's choice in certain action reflects a belief that such action will result in a desired outcome" and that those who believe that greater effort is associated with success and lower effort is associated with higher likelihood of failure are more likely to exert greater effort to master a task [8]. Shweiki et al. argue that if expectancy theory is applied to residency training then more frequent formative assessments will be given, which in turn will foster constant progress toward autonomy and competence and that if improvement in competence is rewarded with autonomy, then resident motivation will increase [8]. This provides another powerful argument for increasing resident autonomy.

\subsection{Cognitive apprenticeship framework}

Wakatsuki et al. interviewed anesthesia residents to find out what their most effective attending teachers in the operating room were doing that others were not and found that fostering autonomy was one of the nine key behaviors these faculty exhibited [10]. This behavior and the other effective teaching behaviors identified in this study fit within the cognitive apprenticeship framework of learning, which includes teaching methods such as scaffolding, modeling, articulation, reflection, and exploration [11]. As Stalmeijer explains, "In cognitive apprenticeship, the cognitive and meta-cognitive (thinking about thinking) processes and skills that experts use when performing a task are emphasized and are the focus of teaching activities" [12]. Cognitive apprenticeship particularly emphasizes using scaffolding to support novice learners as they develop skills that allow autonomous practice of medicine. The steps an educator can take to lead learners through the progression from novice to expert include modeling, coaching, articulation, reflection, and exploration [11]. When modeling for learners, educators should explicitly state what they are demonstrating so that learners do not reach mistaken conclusions about what is being taught. Clinical preceptors should act as a coach by providing real-time feedback and when needed, motivation and mentoring. Clinical educators should articulate their clinical reasoning to help learners develop expert reasoning. Experts should also encourage their trainees to reflect on what they have learned 
from a clinical encounter, how to apply this knowledge to new problems, and how future patients might require a modification in approach. Medical educators also need to allow their learners to explore the limits of their comfort zone so that they can maximize their learning [11].

\section{Ethical considerations}

Training residents involves a delicate balance between appropriate supervision and progressive autonomy to develop the skills and confidence necessary for independent practice. With this in mind, teaching attendings must balance ethical obligations to both their patients and their trainees (and by extension the future patients of their trainees). Other authors have argued that "patient safety goals must account for both short-term outcomes associated with trainees and future outcomes attributable to early career surgeons" [13,14]. Much like the ethical conflicts that arise between respect for a patient's autonomy and beneficence toward that patient, and what offers the best learning opportunity for the resident caring for that patient is sometimes at odds. In situations where patient autonomy and beneficence are in conflict, patient autonomy is felt to take priority over beneficence; however, in the case of patient autonomy and resident education, the decision is not so clear-cut [15].

The first question to consider when weighing the potential ethical dilemma regarding patient safety and resident autonomy is whether increasing resident autonomy impacts patient safety negatively, positively, or indifferently. The published data on this topic is inconclusive. A study analyzing malpractice claims to determine factors that contribute to surgical errors found that interns, residents or fellows contributed to $46 \%$ of surgical errors (attending surgeons contributed to $92 \%$ of surgical errors) and $40 \%$ of cases involved lack of competence in a surgical trainee (whereas $58 \%$ of cases involved lack of knowledge or technical competence by the attending surgeon) [16]. This study also found that $47 \%$ of errors involved inappropriate supervision and in $53 \%$ of cases resulting in patient harm a surgical trainee had the highest or equally highest rate of contribution to the error [16]. A follow up analysis limited to technical errors identified in malpractice claims reported that only $4 \%$ of technical errors were the sole responsibility of a surgical resident or fellow with another $27 \%$ of errors attributable to both an attending surgeon and a trainee; only $9 \%$ of technical errors occurred because of inadequate supervision of trainees [17].

Some studies have suggested that teaching hospitals have higher rates of adverse events than nonteaching hospitals [18] and that surgical complication rates (but not mortality rates) are higher at teaching than nonteaching hospitals [19]. A randomized controlled trial comparing outcomes in a single medical intensive care unit (ICU) for patients treated with nocturnal intensivist staffing to those treated with the daytime intensivist available by phone at night found no significant difference in length of hospital or ICU stay, ICU or in-hospital mortality, rates of ICU readmission, or discharge disposition between the two groups [20]. A systematic review and meta-analysis by Snowdon et al. analyzed studies of clinical supervision of health care professionals and found low quality evidence that clinical supervision of medical professionals (the vast majority were residents) decreased the risk of mortality and complications [21]. This study also found moderate quality evidence that direct supervision of invasive procedures (central venous catheter placement and emergent intubation) resulted in lower rates of complications [21].

Studies examining surgical outcomes with and without residents also have mixed results. Celentano et al. compared outcomes in laparoscopic surgery for 
inflammatory bowel disease for operations which were trainee performed (with either the attending assisting or supervising while unscrubbed in the operating room) or trainer performed (i.e., the attending performed two or more of the critical steps of the operation) and found no significant difference in overall 30-day mortality rate, intraoperative blood loss, reoperation rates, readmission rates, or rates of conversion to an open operation between the two groups [22]. In fact, the only significant difference was an increase in operative time in the trainee group (166.6 vs. 130.4 minutes). In another study post hoc analysis of prospective study data on outcomes of inguinal hernia repairs compared results for hernia repairs performed by junior surgical residents as opposed to those performed by senior surgical residents and found that those performed by junior residents had similar overall complication rates, but increased operative times and higher recurrence rates at 2 years for open, but not laparoscopic repairs [23]. The protocol of this study required the attending surgeon to be scrubbed in from the initial skin incision to the beginning of skin closure, but the degree of involvement of the attending surgeon in performing the critical steps of the operation was not measured. The systematic review and meta-analysis by Snowdon et al. found moderate quality evidence that direct supervision of surgery results in a significant decrease in mortality rates, but low quality evidence that direct supervision did not significantly impact surgical complication rates [21]. This study also found moderate quality evidence that direct supervision decreased the rates of conversion of laparoscopic operations to open operations and evidence that direct supervision did not significantly impact reoperation rates. The meta-analysis, however, does not provide sufficient detail to determine what degree of autonomy the supervised surgical residents in the included studies were granted. A recent report on a resident-run surgical service designed to increase autonomy for senior residents found that patients undergoing cholecystectomy or appendectomy on this service had similar rates of 30-day postoperative complications, readmissions, and major operative adverse events as patients undergoing these operations on other surgical services at the same hospital [23]. The structure of the service was designed so that the supervising attending surgeon scrubbed in for the critical portions of the procedure, but there was no report on how often, if ever, the attending actually performed the critical steps of the operation.

The ACGME, American Colleges of Surgeons (ACS), and the American Medical Association (AMA) all have guidelines specifying that patients must be notified of the roles and level of training of both attending physicians and medical trainees $[3,24,25]$. Despite these guidelines, previous studies have shown that attendings and resident physicians rarely make their roles, level of training, and expected level of participation in procedures or that of their trainees explicitly known to patients [26, 27]. Multiple other studies have shown, however, that patients want to know if residents are going to be involved in their care, particularly if they are going to be involved in an operation they are having [28-30]. Several studies have also found that although the general public is overwhelmingly supportive of physicians-in-training learning through hands on practice, even among patients being treated in teaching hospitals, a significant number of them report they do not want residents involved in their care [31, 32], particularly if that care involves an operation and the resident involved is an intern $[2,29,33]$. Many of these studies have found that the general public has a limited level of understanding of the level of training of residents $[28,31,32]$, but if education is provided about the role of residents and their extent of training, comfort levels with resident participation in their care greatly increases $[28,30]$. 


\section{Traditional methods of granting autonomy}

Traditionally the highest levels of autonomy have been experienced by residents during overnight shifts when there were typically few, if any, attending physicians in the hospital $[14,34,35]$. With the increasing expectations for resident supervision and the decrease in resident work hours, over the last 15 years, the implementation of 24-hour coverage by hospitalists has become more common, resulting in a decrease in resident experience managing patients without direct supervision immediately available; however, this is not uniformly rated by residents as being detrimental to their education $[35,36]$. Another time-honored practice for promoting resident autonomy is the continuity clinic, which has been (and in many cases continues to be) a common part of training for pediatric [37], internal medicine [38], neurology [39], obstetrics and gynecology [40], and even ophthalmology [41] residencies. Although the level of supervision in continuity clinics varies by institution [41], continuity clinics provide the opportunity for residents to be involved in longitudinal care, develop a strong sense of ownership for their patients, and practice some degree of independent decision making.

In years past, senior surgical residents were often allowed to operate with junior residents without attending surgeons physically present in the operating room [27]. This has become a much less frequent occurrence as more stringent requirements for supervision of operations have been adopted and as a result, chief surgical residents have been graduating with significantly fewer teaching assistant cases (cases where the senior resident leads a junior resident through an operation), a trend which began even before the implementation of a universal 80-hour work week for residents in the United States in 2003 [42, 43]. It should be noted, however, that although it is more challenging to have two residents participating in the same operation without risking work hours violations, it is still possible to allow senior residents to operate with junior resident assistants while the attending surgeon is directly supervising (either scrubbed in or present, but not scrubbed). This just requires more restraint on the part of the supervising surgeon. Another, more structured approach to providing surgical residents autonomy is the residentrun surgical service [44]. On such services, the pre- and post-operative care, decisions about when and on whom to operate, and the pre-operative work up are typically managed by senior surgical residents with limited attending involvement. The degree of attending oversight in the operating room may vary, but is typically less hands-on than in most other surgical cases.

\section{Attending responsibilities when granting autonomy}

Supervising attendings have a responsibility to utilize their best judgment about when a trainee has demonstrated the appropriate trustworthiness, level of awareness of his or her limitations, and adequate competence to be given the autonomy to perform a particular patient care task $[45,46]$. Attendings should clearly specify their expectations for residents so that they are aware of the goal they are trying to reach [7]. It is also imperative that supervising physicians provide constructive feedback that is specific enough to allow residents to understand how to improve their performance [46]. The provision of specific and timely feedback may be the most important factor in improving resident performance and is a key component of deliberate practice $[47,48]$. Supervising physicians also have a responsibility to provide appropriate assistance when their trainees reach their limits of ability and/or comfort [46]. Attendings who want to promote resident autonomy should 


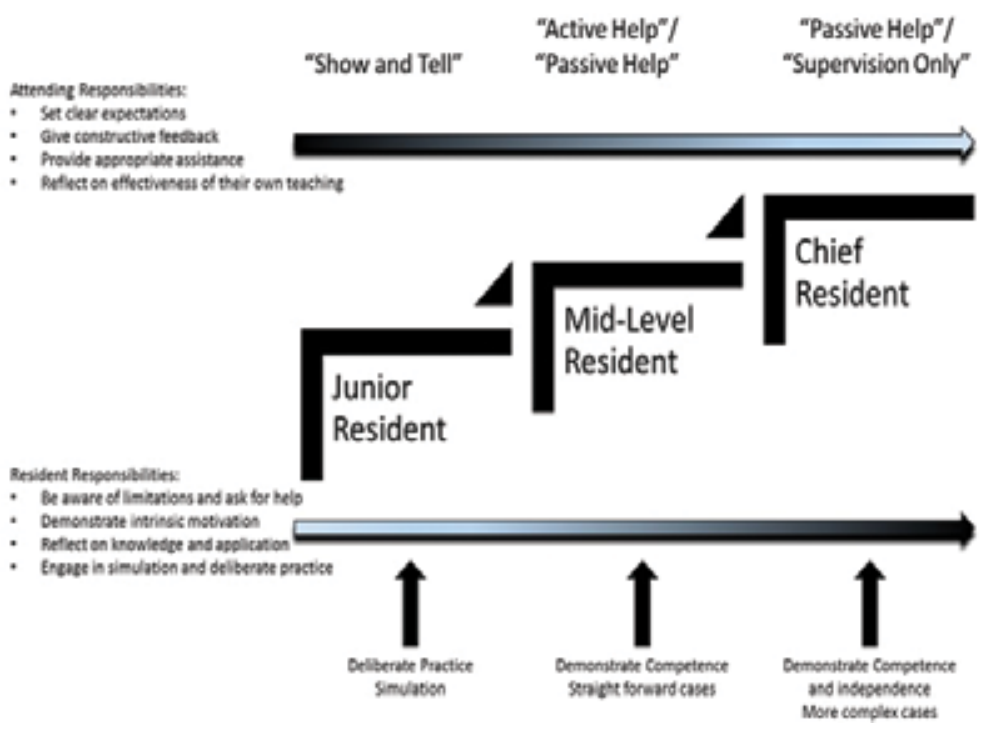

Figure 2.

Schema of graduated autonomy in medical training.

work to provide appropriate scaffolding for novice trainees and to gradually withdraw that scaffolding as the resident develops competence and gains confidence $[7,11]$. Teaching attendings also have a responsibility to their learners to continually reflect on their teaching and its effectiveness and to adopt new techniques that may improve their trainees learning and retention of knowledge and skills (Figure 2).

\section{Trainee responsibilities when being granted autonomy}

Trainees who wish to gain autonomy in patient care have several responsibilities. Perhaps the most essential of these is to develop an awareness of their limitations and a willingness to ask for help when they have reached the limit of their abilities or comfort level [49]. Attendings also want to see trainees demonstrate that they have intrinsic motivation to learn and improve their performance before granting them significant autonomy [7]. Residents should also engage in reflection on both their personal knowledge [50] and how it can be applied to other patients and their performance and how it can be improved. Liability, as determined by courts of law, provides information about the responsibility to which the public and the legal system hold physicians. A study that analyzed court cases where resident physicians were found to have liability found that "the law expects first-year residents to exercise at least that level of knowledge and care expected of other practitioners at a similar state of training or that standard of care applicable to licensed nonspecialists, i.e., general practice doctors" [51]. This study also found that unless residents specifically inform patients of their status as trainees, most courts have ruled that the resident's liability is equivalent to that of licensed physician in their specialty.

Some surgical educators have urged that "the earlier stages of teaching technical skills should take place outside the operating room to permit deliberate practice" [48]. This suggests that residents have a responsibility to ensure that they engage in simulation of basic technical skills with the intent to deliberately practice them until they have achieved proficiency prior to expecting to perform these skills on 
patients. Studies of virtual reality simulation for laparoscopic operations have found that such training decreases error rates and improves efficiency during actual operations $[47,52]$. In fact, some surgical residencies require their residents to demonstrate proficiency of basic technical skills in the simulation lab prior to performing those skills in the operating room [48]. A study that evaluated resident autonomy in over 7000 operations found that the resident's performance in a particular case was the single biggest predictor of how much autonomy the attending granted during that operation [53], which suggests engaging in deliberate practice to improve technical skills is one of the most effective strategies residents can employ to increase their level of operative autonomy. Residents hoping to be granted autonomy during an operation should "hold themselves accountable for entering the operating room as prepared as possible; this should intuitively include review of the patient history" [54]. Preparation for the operating room should also include appropriate knowledge of the relevant anatomy for the case and familiarity with the steps of the operation to be performed. Discussing the surgical plan with the attending prior to surgery is also a valuable step in preparation [54] (Figure 2).

\section{Tools to measure autonomy and competence}

The competence of surgical trainees has historically been assessed in a very subjective manner. The actual performance and competence has been at the discretion of the training program. Traditional methods to assess trainees' competence lie primarily in their fund of knowledge and the ability to pass both qualifying (written) and certifying (oral) board exams. Very little attention has been spent on adequately assessing a trainee's ability to safely and effectively perform various surgical procedures. With the inception of the core competencies set forth by the ACGME there has been an increasing effort to more effectively and objectively assess procedural as well as clinical competence among trainees [55].

The goal of any training program is to graduate competent surgeons who may safely operate independently. However, a prior survey of fellowship directors reported that nearly two-thirds of residents are not able to operate independently for 30 minutes of a major procedure [56]. This issue raises the issue of progressive autonomy in preparation for independent practice. This autonomy must be given in a safe and thoughtful manner to residents that have demonstrated procedural competence. This competence and objective evaluation of performance must be appropriately documented [56]. With that in mind, multiple instruments have been designed to assess competency in the clinical and procedural realms.

Previous utilized approaches to assess operative exposure to various cases have included procedure or case logs. This strategy is highly dependent on timely and accurate recording by the resident. Furthermore, these are often recorded in a retrospective manner and do not allow for fruitful or timely evaluation and more importantly-feedback. Additionally, simply capturing which cases a resident is performing does not adequately capture one's ability or competence in performing a given procedure. Furthermore, previously described and validated tools such as the Objective Structured Assessment of Technical Skills (OSATS) [57] and Multiple Objective Measures of Skill (MOMS) [58] are useful for assessing one's technical skills in a general way but do not adequately assess a trainee's ability to competently complete a specific surgical procedure. This chapter will review several of the most commonly utilized tools to assess competence among surgical trainees. 


\subsection{Entrustable professional activities}

Entrustable professional activities (EPA) are a concept in medical education that allows faculty to make competency-based decisions on the level of supervision for a trainee. EPA's are individual tasks within professional practice that trainees have demonstrated sufficient competence to perform in an unsupervised manner. EPA's are tasks or skills that are able to be executed independently, observable and objectively measurable in process and outcome. These may be introduced in training based on difficulty, degree of risk or complexity in a sequential fashion and may serve as a backbone for graduate medical training. EPA's differ from competencies as EPA's are descriptors of work performed by physicians while competencies are descriptors of the physicians themselves.

Entrustment decisions require more than competence of a particular clinical skill. Trainees must understand their own limitations and know when to ask for help. Entrustment decisions are based on four factors: (1) attributes of the trainee (level of training, confidence, exhausted or not); (2) attributes of the trainers (strict or lenient); (3) context (time of day, resources and facilities available); (4) nature of the EPA (complex vs. straightforward, rare vs. common) [59]. By allowing trainees to perform various clinical tasks or skills, one acknowledges that the trainee has passed a certain threshold allowing for decreased supervision for that particular task or skill. It is recommended that these accomplishments should be documented. One method of documentation is a certificate awarded called a statement of awarded responsibility (STAR) [60].

With EPA's one must ask, "Can we trust this trainee to execute this EPA?" The answer should be translated into five levels of supervision for that EPA (Table 1). The EPA's are translatable for all specialties within medicine and may be tailored to individual specialty needs for trainees. The EPA's are currently in development for multiple medical specialties but the formula for their development is generalizable and should be based on the specialty's requirements and milestones. Faculty development in providing EPA-based assessments is paramount. The decisions based on these EPA's may serve as a "license" for trainees to perform a specific EPA with the appropriate level of supervision [59].

\subsection{The superb/safety model}

In an effort to optimize resident supervision in non-procedural situations (established within general internal medicine) a bidirectional model SUPERB/SAFETY was developed by Farnan et al. [61]. The components of the acronym are as follows: Set expectations for when to be notified; Uncertainty is a time to contact; Planned communication; Easily available; Reassure fears; and Balance supervision and autonomy. Those for SAFETY include: Seek attending physician input early; Active clinical decisions; Feeling uncertain about clinical decisions; End-of-life care or family/legal issues; Transitions of care; and You need help with the system/hierarchy [61].

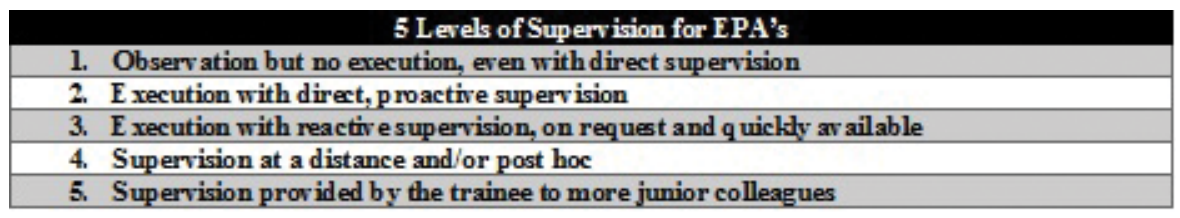

Table 1.

Entrustable professional activities. 
The SUPERB/SAFETY model is developed off of qualitative data and demonstrates the specific actions necessary for optimizing clinical care in parallel with ensuring effective education and development of residents. This is referred to as a bidirectional model in that both the resident and faculty members must take an active role in the supervisory relationship. This model helps to establish that expectations are clear, communication is available and timely and that autonomy is appropriate with providing adequate resident supervision [61].

\subsection{Resident supervision index}

A group of scholars from Graduate Medical Education (GME) and the Institute of Medicine have come together to identify "optimal" level of supervision of trainees. This definition of what optimal means in graduate medical education and supervision is paramount to not only optimize trainee development but also to ensure patient safety. In order to accomplish this, the Resident Supervision Index (RSI) was developed to measure the intensity of resident supervision during outpatient clinical encounters. The RSI is comprised of (1) the RSI inventory-a validated and reliable instrument $[62,63]$ to be completed by faculty and residents to assess supervision data on outpatient care encounters [62, 64]; and (2) RSI scores are calculated from the RSI inventory to quantify the intensity of supervision of residents [65].

The RSI theory rests on several theoretical assumptions regarding patientcentric optimal resident supervision. This model is based on supervision as "resource allocation of scarce clinic resources, including residents, so as to maximize the collective health outcomes of all clinic patients seen in the teaching clinic" [65]. The explicit theoretical assumptions include: optimal supervision, informed decision, patient assignment, professional time, patient outcomes and resident learning (Table 2).

Theoretical results derived mathematically include: optimal supervision identified-when the attending physician supervises residents in a way that allows those that supervise to allocate time among patients and between patient care and supervision to achieve the greatest effect on patient outcome. This theory is centered on resource allocation to achieve a single goal of optimal patient outcomes. There must be a balance between being under-supervised and over-supervised, where residents miss appropriate clinical activities that would not have resulted in adverse patient outcomes. Optimal patient outcomes and progressive autonomy for residents are theoretically compatible. The RSI establishes a framework for scientific research to more accurately measure the connection between resident supervision and patient outcomes and may be very useful in informing GME policies related to appropriate resident autonomy [65].

\subsection{Ottawa surgical competency operating room evaluation (O-SCORE)}

The O-SCORE (Table 3) is a tool that was designed as a succinct instrument to assess competence on any surgical procedure. In the development of the O-SCORE, four surgeons, two evaluation experts and one psychometric researcher considered all of those features of any surgical procedure that are necessary to assess competence. They defined surgical competence as "readiness for independent performance of the procedure." The key to the O-SCORE's assessment method is to evaluate a trainee's readiness for independent performance for a given procedure rather than comparing that trainee to a peer group. The goal of this design was to force raters away from a central scoring tendency. The evaluation was based on a trainee's degree of "active participation" in each key aspect of a given procedure [55]. 


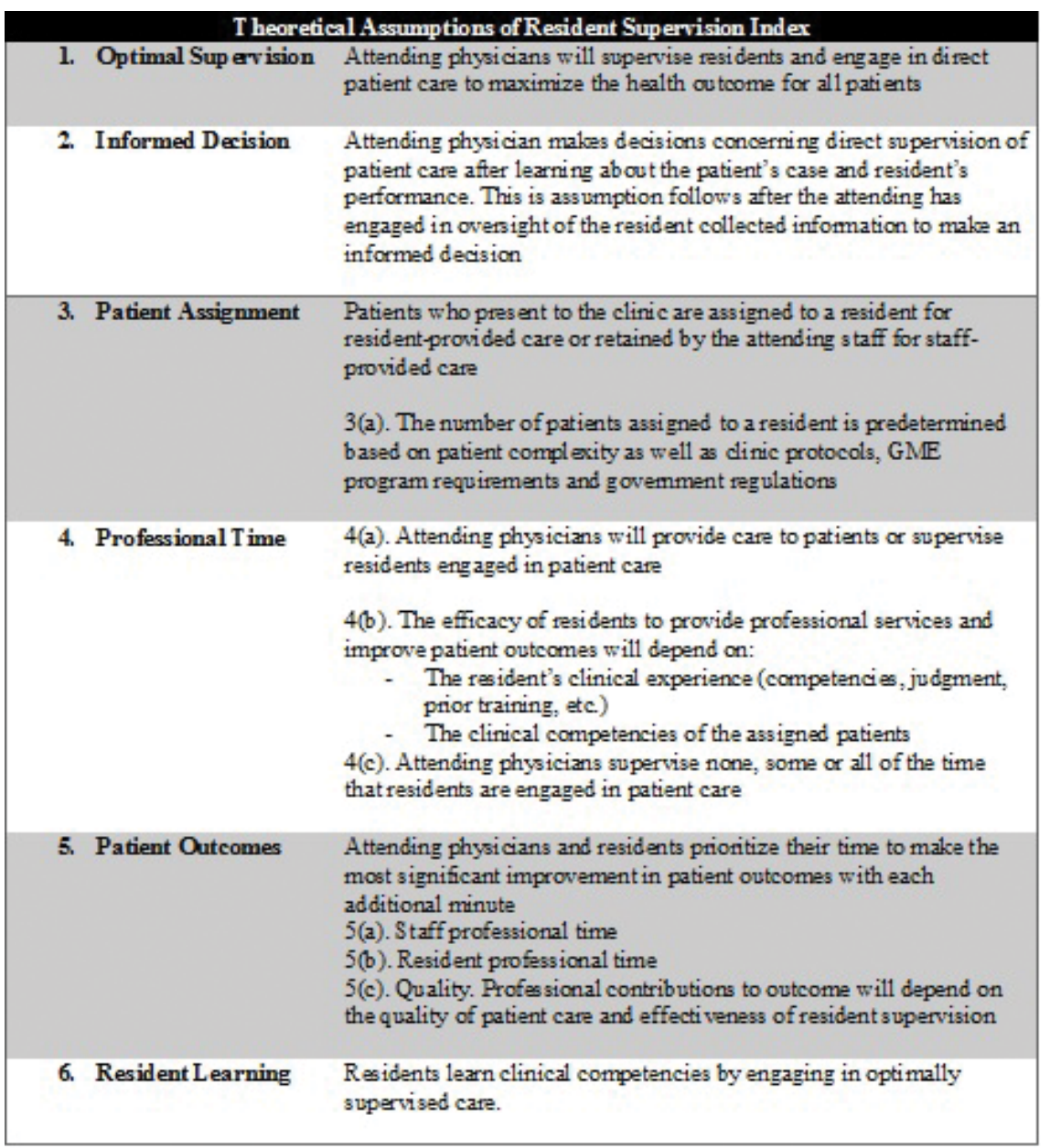

Table 2.

Resident supervision index: Adapted from Kashner et al. Journal of Graduate Medical Education [65].

The first version of the O-SCORE consisted of a 14-item instrument that consisted of 10 items rated on a 5-point scale, 2 yes/no questions and 2 open-ended questions (one specific aspect of the case performed well and one that requires improvement). This was later refined to 11 items ( 8 items rated on the 5-point competency scale, 1 yes/no question about the competency to perform the procedure independently and the same 2 open-ended questions). While initially piloted amongst orthopedic surgery trainees, this was expanded across specialties to include general surgery. The O-SCORE was used to evaluate residents' performance with five common general surgery procedures: open hernia repair, laparoscopic appendectomy, laparoscopic cholecystectomy, emergency laparotomy and axillary node dissection [55].

The O-SCORE was able to accurately differentiate amongst senior, midlevel and junior trainees. There was a trend towards improvement in performance with several procedures with increasing PGY-levels. While this evaluation instrument was only assessed in two surgical specialties, the O-SCORE is felt to provide an objective and reliable assessment of competence for perioperative decision-making and procedural competency [55]. 


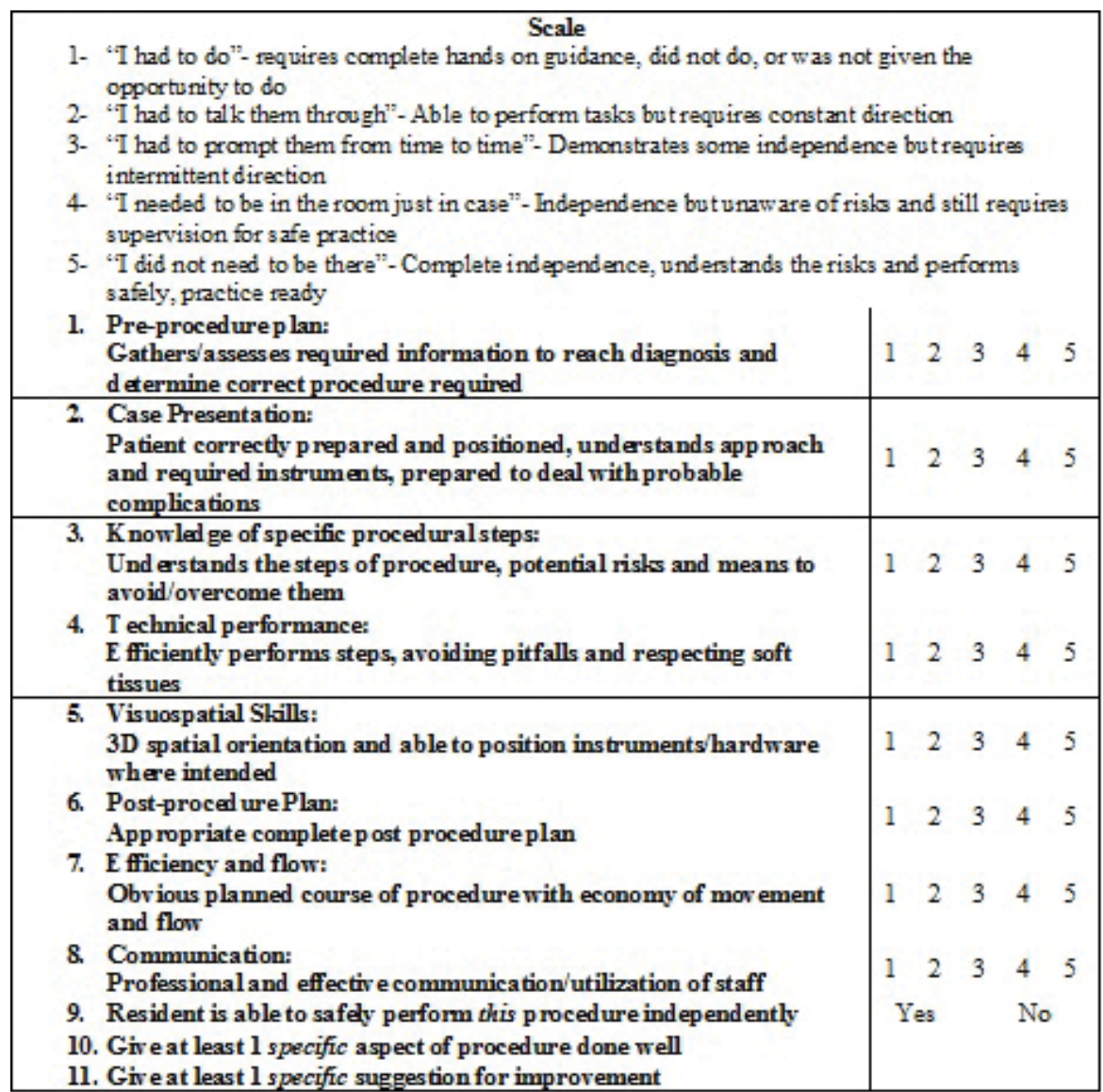

Table 3.

Ottawa surgical competency operating room evaluation (O-SCORE): Adapted from Gofton et al. Academic Medicine, $2012[55]$.

\subsection{Zwisch scale}

The Zwisch scale was initially described by DaRosa et al. in 2013 [56, 66] in which the authors describe a 1-dimensional behaviorally anchored ordinal scale used to assess the amount of guidance provided by an attending surgeon provides to the trainee during the "critical portion" of a procedure (Table 4). This 4-level scale describes the spectrum of assistance required by the attending surgeon ranging from "show and tell" in which the attending surgeon performs the critical portions of a case and explains each step of the procedure to the resident to "supervision only"-the most advanced level — where the attending surgeon is present only to guarantee patient safety. The middle levels consist of "active help" and "passive help." These levels are coded as 1 through 4, with supervision only receiving a level of 4 [56].

The Zwisch scale was compared to modified versions of the OPRS and O-SCORE instruments. The study by George et al. looked at 1490 operative performance assessments for 31 residents across several surgical procedures, with laparoscopic cholecystectomy and laparoscopic appendectomy being the most common. The authors demonstrated a significant increase in Zwisch scores across increasing PGYlevels. Interestingly, among PGY-5 residents, only 23.2\% of the observed operations were scored at level 4 (supervision only). Additionally, the Zwisch scale correlated closely with the operative volume of a given procedure for each resident. If a 


\begin{tabular}{|c|c|c|}
\hline Stage of Supervision & Attending Behaviors & $\begin{array}{c}\text { Resident Behaviors with Level } \\
\text { of Supervision }\end{array}$ \\
\hline Show and Tell & $\begin{array}{l}\text {-Does majority of key portions } \\
\text { of the case } \\
\text { - Narrates steps of the case } \\
\text {-Demonstrates key concepts and } \\
\text { skills }\end{array}$ & $\begin{array}{l}\text {-Opens and closes } \\
\text {-First assists and observes }\end{array}$ \\
\hline Active Help & $\begin{array}{l}\text { Shifts between and sorgeon and } \\
\text { firt assist roles } \\
\text {-As first assist, actively as sists } \\
\text { resident in surgeon role } \\
\text {-Coaches for specific technical } \\
\text { skills } \\
\text {-Identifies anatomical landmarls } \\
\text { for the resident }\end{array}$ & $\begin{array}{l}\text { Shifts between surgeon and } \\
\text { first assist roles } \\
\text {-Knows all the needed technical } \\
\text { skills } \\
\text {-Demonstrates increased ability } \\
\text { to perform different key parts of } \\
\text { an operation with assistance }\end{array}$ \\
\hline Passive Help & $\begin{array}{l}\text {-Assists and follows the lead of } \\
\text { the resident } \\
\text {-Coaching regarding refinement } \\
\text { of skills and techniques }\end{array}$ & $\begin{array}{l}\text {-Able to "set up" and } \\
\text { accomplish next step for the } \\
\text { entire case with increasing } \\
\text { efficiency } \\
\text {-Recognizes critical transition } \\
\text { points }\end{array}$ \\
\hline Supervision only & $\begin{array}{l}\text {-Provides no unsolicited advice } \\
\text {-Assisted by a junior resident or } \\
\text { attending who acts like a junior } \\
\text { resident } \\
\text {-Monitors progress and ensures } \\
\text { patient s afety }\end{array}$ & $\begin{array}{l}\text {-Able to work with } \\
\text { inexperienced first assistant } \\
\text {-Can safely complete a case } \\
\text { without faculty assistance } \\
\text {-Able to recover from most } \\
\text { errors } \\
\text { - Recognizes when to seek } \\
\text { advice/help }\end{array}$ \\
\hline
\end{tabular}

Table 4.

Zwisch scale: Adapted from DaRosa et al. Journal of Surgical Education, 2013 [66].

resident performed 5 or fewer procedures of a given type, the median Zwisch scale was "active help" while if more than 5 had been performed prior to evaluation with the Zwisch scale the median score was "Passive Help" [56].

The Zwisch scale was highly correlated with other assessment tools such as the OSCORE and OPRS as assessed by video rating by blinded reviewers. These results come to mean that the Zwisch scale can be used to accurately assess intraoperative performance. Additionally there was noted to be high inter-rater reliability. Interestingly, there was significant reliability amongst "types" of reviewers (attending physicians, in-room observer, and video raters) giving the Zwisch scale the flexibility of not needing to be used physically in the operating room as long as the audio-visual recording of the faculty-resident interaction is able to be reviewed [56].

The simplicity of the Zwisch scale with its 4-point scale and its accessibility on a smart-phone app allow for real-time accessibility and promotes a significantly higher response rate [67]. Limitations of the Zwisch scale include it being limited to a summative assessment tool. It does not provide the more granular information that is provided by other evaluation tools such as the OPRS. Despite these limitations, the Zwisch scale allows for an accurate and reliable measure of resident operative performance and the amount of faculty guidance for a given procedure and allows for a longitudinal resident operative performance evaluation method.

\subsection{Procedural autonomy and supervision system (PASS)}

PASS is a smartphone-based app that utilizes the Zwisch scale and a scale of procedural difficulty. With this instrument, the faculty member receives an 
evaluation prompt through the smartphone. The faculty member must then enter the procedure that was performed, the date and time and resident name. Then the faculty member scores the resident performance using the Zwisch scale and then scores the difficulty of that particular procedure as easiest $1 / 3$, middle $1 / 3$ or most difficult $1 / 3$ for a particular procedure [68].

The PASS app was designed to encourage the completion of operative performance assessments in a timely and efficient manner that minimizes disruptions in a busy surgical practice. In fact, a recent study by Fryer et al. demonstrated no increase in mean OR times while using PASS. Additionally, there was no significant difference in OR satisfaction amongst residents. However, depending on statistical methods used, the authors did identify a slight decrease in positive responses in the OR Educational Environment Measure (OREEM) scale. In their assessment, faculty reported a lesser degree of agreement with four specific items on the OREEM scale: (1) my resident(s) and I got along well; (2) the atmosphere in the OR was pleasant; (3) the staff in the OR was friendly to my resident(s); and (4) my resident(s) felt like part of a team in the OR; following implementation of PASS. While one may ponder reasons for this difference, including greater anxiety amongst faculty members as they work with residents who wish for more participation during a given procedure; a less relaxed environment knowing that an imminent evaluation was looming; or there may have been a sense of a more formal mentor-trainee relationship that may have led to this perceived decrease in faculty satisfaction [68].

Three additional OREEM items that prompted more negative responses by faculty after PASS implementation were related to residents' skills. This difference may be related to a greater awareness of a given resident's operative performance that led to a more critical evaluation of performance. Thus, using this PASS and the built-in Zwisch scale, there may have been an inherent change in the way a faculty member perceived a resident's operative performance [68].

\subsection{Global operative assessment of laparoscopic skills (GOALS)}

GOALS is an assessment instrument that evaluates performance during laparoscopic procedures over five domains. It was initially validated using common laparoscopic procedures-laparoscopic appendectomy and cholecystectomy. Others have worked to validate this instrument in other procedures including both groin and incisional hernias. GOALS assesses performance over five domains (depth perception, bimanual dexterity, efficiency, tissue handling, and autonomy). For each of these five domains, there is a rating from 1 to 5 with a descriptive anchor for scores 1 , 3 and 5 . A total score for each operation is calculated by adding the scores from all five domains and is used as an overall assessment of a trainee's performance [69].

While GOALS provides formative feedback across multiple domains, the domain of autonomy assesses the degree to which the trainee completed a given procedure with varying amounts of supervision. A score of 1 means that the trainee was "unable to complete the entire procedure, even in a straightforward case and with extensive verbal guidance." A score of 3 states that the trainee is "able to complete operation safely with moderate prompting" while a score of 5 is "able to complete operation independently without prompting" [69]. The combination of domains that evaluate a trainee's operative skills (depth perception, bimanual dexterity and tissue handling) along with the degree to which a trainee required supervision and intervention by an attending surgeon is critical for a more complete assessment of one's ability to perform a given procedure. Thus GOALS may be useful in assessing a trainee's progress across their time in training as well as identify specific areas of improvement required to attain adequate technical proficiency. 


\section{Outcomes associated with autonomy}

Across disciplines, patient safety and providing high quality care are the priorities within any health system. This is balanced with providing appropriate trainee supervision to allow for adequate education and resident development. Concerns that center on increasing regulations of resident education including limited work hours may hinder residents' training experience and an appropriate level of autonomy [70-73]. Studies across many disciplines of medical training have studied the effects of autonomy among trainees on patient outcomes from in-patient pediatrics, anesthesia as well as surgery. While there are studies that demonstrate less than ideal outcomes related to longer operative times [74], or overall worse outcomes, many studies have demonstrated significant improvement in resident satisfaction and confidence without any detrimental effects on patient outcomes.

Biondi et al. hypothesized that modifying the extent of attending input during the pediatric admissions process would improve efficiency in the admission process, increase perceptions of patient ownership without compromising the quality of patient care. In this study, a new process was implemented regarding pediatric admissions in which the ED physician called the pediatric admitting resident (PGY-3 or 4 pediatrics resident) directly. In this new process the admitting resident was empowered to accept the patient and perform necessary admission duties. The patient was evaluated by the attending on the day of admission during daytime hours (7 am-5 pm). However, during evening hours ( $5 \mathrm{pm}-10 \mathrm{pm})$, the resident was expected to evaluate the patient and staff the patient with the attending after developing a plan. During overnight hours (10 pm-7 am), the resident contacted the attending at the resident's discretion and the patient was seen and evaluated by the attending in the morning. The residents were encouraged to contact the overnight attending on call if they had questions or felt the admission was inappropriate [70].

This new process resulted in a reduction in the time from the initial request by the ED for admission to the time the admission order was placed by over an hour. While there was improved efficiency in the admission process there were several cases noted where the resident's plan did not meet the standard of care. However, due to the relative low frequency of these events there was not identifiable impact on major patient outcomes [70]. This study was likely underpowered to truly detect a difference in the infrequent quality of care and patient harm events. Despite the limitations outlined by the authors, they concluded increased resident autonomy by limiting mandatory attending input at the time of admission improves efficiency and does not adversely affect major patient outcomes.

Several published studies have studied outcomes associated with appendectomies performed by general surgery residents compared to those performed by senior surgeons (attendings). One such study by Siam et al. [75] demonstrated no difference in outcomes for those appendectomies performed by senior general surgeons and general surgery residents. In this large retrospective study from Israel, over 1600 appendectomies were evaluated with over 500 of those performed alone by a general surgery resident. As mentioned in an earlier section, Siam et al. did demonstrate a longer length of procedure time in procedures performed by residents as well as a higher rate of open appendectomies compared to those performed by attending surgeons. However, there was no difference in post-operative complications or hospital length of stay between the two groups. Thus, the authors concluded that it is safe to allow surgical residents to perform appendectomies done under standard conditions to be safe. This is balanced with the residents' ability to recognize and engage an attending should such a clinical situation arise [75]. Other studies have similarly demonstrated safety in resident performed appendectomies with minimal attending supervision [76-79]. 
This idea of safe and effective resident autonomy amongst surgical subspecialties may translate to safe and quality patient care in the clinic setting as well. A study by Day et al. demonstrated progressive surgical autonomy amongst plastic surgery residents in a plastic surgery resident clinic (PSRC). The PSRC was staffed by attending plastic surgeons, but all aspects of patient care history and physical exams and interpretation of radiology studies were performed by residents and a supervising chief resident. In this study, over 3300 clinic appointments and 653 operations were performed and there was a statistically significant increase in resident autonomy as one progressed through post-graduate years. Not only did this study confirm improved autonomy with the PSRC model, but it also demonstrated improved continuity of patient care amongst residents, one facet of training threatened by the duty hour regulations. Furthermore, graduated chief residents were surveyed and reported significant value in the surgical experience, operative autonomy, medical knowledge and ACGME core competencies afforded by supporting a resident run clinic. These findings have been echoed in other studies as well in the settings of a resident-run aesthetic clinic [80] which demonstrated a structured, autonomous experience in rhinoplasty with acceptable complication rates as well as good satisfaction as rated by both patients and residents [81].

Additional studies have been performed in the realm of plastic surgery, specifically cosmetic surgery. A retrospective chart review by Walker et al. [82] of 326 patients with 714 aesthetic procedures demonstrated that a chief resident run clinic allowed the chief residents to feel "very comfortable" based on survey results performing facelifts, body contouring and aesthetic breast surgery. None of the residents who responded completed a cosmetic fellowship with $60 \%$ of the residents surveyed stating that the experience from the chief led clinic contributed to their decision to not pursue further cosmetic training. The authors demonstrated that a chief-run clinic with the appropriate supervision and autonomy allows chief residents to feel comfortable performing common cosmetic procedures. They conclude that chief resident clinics may provide good results with no increase in complications rates or need for revisions. Additionally, it significantly improved resident comfort levels with common procedures and may even mitigate the need for graduating chief residents to pursue further training in aesthetic fellowships.

Despite the challenges (legal, regulatory, staffing) of establishing a chief resident run service or clinic in any specialty, recent studies by Jarman et al. [44, 83, 84] demonstrated that this can be done safely and effectively in the realm of general surgery. Chief resident run services afford chief residents the opportunity to provide and hone perioperative decision making and hone operative skills on common general surgical procedures with appropriate supervision-most often in the "passive help" or "supervision" roles only as determined by the Zwisch scale. Jarmen et al. demonstrated a strong correlation between procedures performed on the chief resident service and during their first year of clinical practice. While this study did not measure the quality or rates of complications associated with a chief-run service, there was significant increase in positive survey results by the graduated chief residents who participated in the chief resident service and the autonomy that the service afforded them [85]. Wojcik et al. [84] demonstrated that implementation of a resident-run minor surgery clinic safe in regards to 30-day post-procedure complications which persisted on multivariate analysis. Addition to demonstrating patient safety, residents evaluated the clinic experience in a very positive manner, increased operative autonomy being the biggest strength cited.

Another measure to assess for autonomy in the operating room is based on the volume of teaching assistant (TA) cases. As eluded earlier, it has been demonstrated that TA cases have declined by 79\% between the years of 1999 and 2012. A study by Kantor et al. [42, 83, 86] sought to implement a resident acute care surgery 
consult service (RACS) and to assess the impact of resident autonomy as demonstrated by TA cases by chief residents. Over a 12-month period, over 1100 consults were seen with nearly one third of those being operative. For appropriate operative cases, $82 \%$ of the cases were done entirely by residents. Using graduating chief residents prior to implementation of RACS as controls, the number of TA cases after RACS more than doubled (mean 13.4-32.2) per chief resident over the period of 1 month. Along with increased TA cases, the authors demonstrated an increase in resident satisfaction with case complexity and variety. Furthermore, there was improved efficiency with operative consultation times [83].

Autonomy both in and out of the operating room is necessary to allow surgical trainees in all surgical specialties to become confident in their operative skills and decision-making. While there are studies that demonstrate less than ideal outcomes related to longer operative times, many studies have demonstrated significant improvement in resident satisfaction and confidence without any detrimental effects on patient outcomes. Trainees must be afforded the right balance of autonomy and supervision to ensure appropriate growth of technical and non-technical skills for their given field of specialization but also to ensure appropriate decision making and patient safety.

\section{Encouraging autonomy}

In the face of increased scrutiny of decreased resident work hours and an increasing amount of administrative responsibilities placed on residents in this day and age, residents and their supervising faculty must strike a balance between maximizing education in a limited timeframe, a gradual and appropriate increase in autonomy across the spectrum of patient care and quality of care and patient safety. There are some attributes that are harder to objectively measure that may indicate a resident's investment or "ownership" for a patient. Ownership, as defined by McLaren et al. [87] is a broad term that includes professionalism, patient care and patient safety. It is this ownership and its perceived implications upon which many faculty members may grant increased autonomy. A recent qualitative study by Chen et al. demonstrated that willingness to grant a resident autonomy may hinge on various resident characteristics, medical knowledge and factors that go beyond the current OR case [87].

Resident characteristics that may allow an attending to offer more autonomy include the resident's level of training, the amount of personal effort the resident placed into preparing for a given case, the resident's attitude and motivation as well as the perceived level confidence by the resident. Medical knowledge is based on several different aspects that may be assessed even before the safety time-out is performed for a particular case. First is the resident's basic knowledge of anatomy, pathology and the steps of the procedure. A resident is more likely to gain autonomy if he/she is able to understand the attending surgeon's guidance and directions. Taking this assessment of knowledge one step further is whether the resident is able to develop an appropriate operative plan, demonstrate adequate judgment and know what instruments are needed for the next step in an operation. This is further supported by the resident's ability to actively teach medical students and other residents these steps while performing the procedure. Autonomy is also guided by experience with that resident that goes beyond that of the current surgical case. These experiences may be the result of prior experiences that the attending surgeon has had with a particular resident, the reputation of a resident from co-residents, chief residents and other faculty members who have worked with that resident. Furthermore, objective evaluations and milestone documentation also often play a 
role in one's ability to gain autonomy in the operating room. An attending surgeon's teaching philosophy and the contextual issues about the case (case complexity) also will determine the amount of autonomy a resident may be allowed to have during a case [88].

Faculty development efforts are paramount to optimizing the assessment and appropriate granting of autonomy for residents among attending surgeons. Faculty development would allow for expert surgical teachers to educate other faculty members as to their best practices. One example of a best practice as outlined by Chen et al. is the set of questions (Table 5) "Performance-ImpressionCharacteristics-Knowledge-Situation" (PICKS) that will allow an attending to quickly and easily assess a resident's readiness for autonomy in the operating room, even prior to the safety timeout [88].

Optimizing resident autonomy is dependent on development of both the trainer (attending surgeon) and trainee (resident). Faculty development efforts that teach attending surgeons to assess and guide the appropriate level of autonomy based on the three groups of evidence (resident characteristics, medical knowledge and factors beyond the current OR case) as outlined by Chen et al. as well as the context variable of a specific surgical case can assure more appropriate levels of autonomy for trainees. Similarly, teaching residents to demonstrate increased medical knowledge and preparation for the surgical operation to the attending surgeon will also help to optimize the autonomy they are granted in the operating room [88].

Other strategies to promote autonomy as described by Beck et al. [89] on Family-Centered Rounds (FCR) may be broken down to various phases of the FCR activity. Prior to the beginning of FCR, framing expectations and agreeing upon nonverbal signals were found to be helpful. Setting expectations allows for defining roles of team members and allows the attending to be viewed primarily as a consultant who may be allowed to step in based on the predetermined nonverbal

\begin{tabular}{|c|c|}
\hline P-Performance & Do I feel comfortable with this resident's technical performance and skills? \\
\hline I -Impression & $\begin{array}{l}\text { What is my perception of this resident? } \\
\text { - Did the resident meet my expectation in recent cases? } \\
\text { - Does the resident have a good repu tation among faculty, residents and } \\
\text { patients? } \\
\text { - Does the resident do well in floor management? }\end{array}$ \\
\hline C-Characteristics & $\begin{array}{l}\text { What is the resident's training level (PGY-year)? } \\
\text { Has this resident done this procedure before? } \\
\text { Has the resident prepared for this case? } \\
\text { - Does the resident know ny patient (history, imaging, and previous } \\
\text { surgeries)? } \\
\text { - Does the resident know this procedure (indications and } \\
\text { - Domplications)? }\end{array}$ \\
\hline K - Knowledge & $\begin{array}{c}\text { Does this resident's knowledge and operative plan fit my case? } \\
\text { - Does the resident understand my instructions? } \\
\text { - Does the resident have a reasonable operative plan }\end{array}$ \\
\hline S- Situation & $\begin{array}{l}\text { Do I need to speed up this case? } \\
\text { - Is this case too complicated for this resident? } \\
\text { - Do I have a tight schedule today? }\end{array}$ \\
\hline
\end{tabular}

Table 5.

Performance-impression-characteristics-knowledge-situation (PICKS): Adapted from Chen et al. Journal of Surgical Education. 2017 [88]. 
signals. A pre-rounds huddle allows the resident to address any major clarifying questions and allows the resident to develop the logistical and educational aspects of the FCR. During the FCR, deliberate positioning of the senior resident and establishing them as the primary physician so that questions and issues are directed primarily to them. Allowing for flexibility and allowing for the resident to develop their own plan so long as to prevent patient harm and avoiding micro-management of those decisions will also be paramount to promoting autonomy in these situations. If a resident does not initially come up with a plan, the attending should probe and encourage the resident to develop a plan. Additionally, maintaining silence and allowing the resident to control rounds will encourage autonomy for the resident. After the FCR, promoting reflection and allowing the resident to reflect on how the rounds went is critical to improvement. Also allowing the resident to offer feedback to junior residents and medical students will promote the educational value of the FCR [89]. These steps outlined by Beck may be widely translatable to other activities within graduate medical education across specialties.

\section{Conclusion}

Training the next generation of physicians to be competent and independent physicians is paramount to ensure adequate experience and patient safety as they prepare to enter independent practice. This has become more difficult in the face of increasing regulatory guidelines and restricted work hours. The traditional models of promoting resident autonomy in training have been challenged over the past several years and require innovative solutions as outlined in this chapter. The responsibility for promoting and earning autonomy rests equally with attendings and trainees. These theories and methods of promoting and granting autonomy continue to evolve and must be objectively studied to ensure effectiveness and safety that we owe our patients. Efforts to optimize autonomy must continue to be a focus of graduate medical education.

\section{Conflict of interest}

The authors declare that they do not have any conflicts of interest to declare in the authorship of this book chapter.

\section{Author details}

Amanda Cooper and Steven Allen*

Penn State Milton S. Hershey Medical Center, Hershey, PA, United States

*Address all correspondence to: sallen4@pennstatehealth.psu.edu

\section{IntechOpen}

(C) 2019 The Author(s). Licensee IntechOpen. This chapter is distributed under the terms of the Creative Commons Attribution License (http://creativecommons.org/licenses/ by/3.0), which permits unrestricted use, distribution, and reproduction in any medium, provided the original work is properly cited. (cc) BY 


\section{References}

[1] Hinchey KT et al. "I can do patient care on my own": Autonomy and the manager role. Academic Medicine. 2009;84(11):1516-1521

[2] Kempenich JW et al. How do perceptions of autonomy differ in general surgery training between faculty, senior residents, hospital administrators, and the general public? A multi-institutional study. Journal of Surgical Education. 2015;72(6):e193e201

[3] Common Program Requirements. 2011. Available from: http://www.acg me.org/Portals/0/PDFs/Common_Prog ram_Requirements_07012011\%5B2\% 5D.pdf [Cited Sep 23, 2018]

[4] Kohn LT, Corrigan JM, Donaldson MS, editors. To Err Is Human: Building a Safer Health System. Washington, DC: National Academy Press, Institute of Medicine; 1999

[5] Ryan RM, Deci EL. Selfdetermination theory and the facilitation of intrinsic motivation, social development, and well-being. The American Psychologist. 2000;55(1): 68-78

[6] Ten Cate TJ, Kusurkar RA, Williams GC. How self-determination theory can assist our understanding of the teaching and learning processes in medical education. AMEE Guide No. 59. Medical Teacher. 2011;33(12): 961-973

[7] Hoffman BD. Using selfdetermination theory to improve residency training: Learning to make omelets without breaking eggs. Academic Medicine. 2015;90(4): 408-410

[8] Shweiki E et al. Applying expectancy theory to residency training: Proposing opportunities to understand resident motivation and enhance residency training. Advances in Medical Education and Practice. 2015;6:339-346

[9] Wigfield A, Eccles JS. Expectancyvalue theory of achievement motivation. Contemporary Educational Psychology. 2000;25(1):68-81

[10] Wakatsuki S et al. What makes for good anesthesia teaching by faculty in the operating room? The perspective of anesthesiology residents. Cureus. 2018; 10(5):e2563

[11] Merritt C et al. A cognitive apprenticeship-based faculty development intervention for emergency medicine educators. The Western Journal of Emergency Medicine. 2018;19(1):198-204

[12] Stalmeijer RE. When I say ... Cognitive apprenticeship. Medical Education. 2015;49(4):355-356

[13] George BC, Dunnington GL, DaRosa DA. Trainee autonomy and patient safety. Annals of Surgery. 2018;267(5):820-822

[14] Halpern SD, Detsky AS. Graded autonomy in medical educationManaging things that go bump in the night. The New England Journal of Medicine. 2014;370(12):1086-1089

[15] Scarlet A et al. Respect for whose autonomy? Communicating with patients regarding trainee involvement. Bulletin of the American College of Surgeons. 1 August 2018

[16] Rogers SO Jr et al. Analysis of surgical errors in closed malpractice claims at 4 liability insurers. Surgery. 2006;140(1):25-33

[17] Regenbogen SE et al. Patterns of technical error among surgical malpractice claims: An analysis of strategies to prevent injury to surgical 
patients. Annals of Surgery. 2007; 246(5):705-711

[18] Brennan TA et al. Hospital characteristics associated with adverse events and substandard care. The Journal of the American Medical Association. 1991;265(24):3265-3269

[19] Khuri SF et al. Comparison of surgical outcomes between teaching and nonteaching hospitals in the Department of Veterans Affairs. Annals of Surgery. 2001;234(3):370-382; discussion 382-3

[20] Kerlin MP et al. A randomized trial of nighttime physician staffing in an intensive care unit. The New England Journal of Medicine. 2013;368(23): 2201-2209

[21] Snowdon DA et al. Does clinical supervision of health professionals improve patient safety? A systematic review and meta-analysis. International Journal for Quality in Health Care. 2016; 28(4):447-455

[22] Celentano V et al. Safety of supervised trainee-performed laparoscopic surgery for inflammatory bowel disease. International Journal of Colorectal Disease. 2015;30(5):639-644

[23] Wilkiemeyer M et al. Does resident post graduate year influence the outcomes of inguinal hernia repair? Annals of Surgery. 2005;241(6): 879-882; discussion 882-4

[24] American College of Surgeons. Statements on Principles. 2016. Available from: http://www.facs.org/ab out-acs/statements/stonprin-ivb [Cited Sep 26, 2018]

[25] Association AM. Available from: https://www.ama-assn.org/deliveringcare/truth-advertising [Cited Sep 26, 2018]

[26] Santen SA, Rotter TS, Hemphill RR. Patients do not know the level of training of their doctors because doctors do not tell them. Journal of General Internal Medicine. 2008;23(5):607-610

[27] Knifed E, Taylor B, Bernstein M. What surgeons tell their patients about the intraoperative role of residents: A qualitative study. American Journal of Surgery. 2008;196(5):788-794

[28] Knifed E, July J, Bernstein M. Neurosurgery patients' feelings about the role of residents in their care: A qualitative case study. Journal of Neurosurgery. 2008;108(2):287-291

[29] Porta CR et al. Training surgeons and the informed consent process: Routine disclosure of trainee participation and its effect on patient willingness and consent rates. Archives of Surgery. 2012;147(1):57-62

[30] Kempenich JW et al. The effect of patient education on the perceptions of resident participation in surgical care. Journal of Surgical Education. 2016; 73(6):e111-e117

[31] Hemphill RR et al. Patients' understanding of the roles of interns, residents, and attending physicians in the emergency department. Academic Emergency Medicine. 1999;6(4): 339-344

[32] Santen SA et al. Do patients understand their physician's level of training? A survey of emergency department patients. Academic Medicine. 2004;79(2):139-143

[33] Cowles RA et al. Doctor-patient communication in surgery: Attitudes and expectations of general surgery patients about the involvement and education of surgical residents. Journal of the American College of Surgeons. 2001;193(1):73-80

[34] Kerlin MP, Halpern SD. Twentyfour-hour intensivist staffing in teaching hospitals: Tensions between 
safety today and safety tomorrow. Chest. 2012;141(5):1315-1320

[35] Greene JG. Neurohospitalists enhance resident perception of the educational and clinical value of a night float rotation. Neurohospitalist. 2013; 3(4):179-184

[36] Trowbridge RL et al. The effect of overnight in-house attending coverage on perceptions of care and education on a general medical service. Journal of Graduate Medical Education. 2010;2(1): 53-56

[37] Rosenberg AA et al. Training gaps for pediatric residents planning a career in primary care: A qualitative and quantitative study. Journal of Graduate Medical Education. 2011;3(3): 309-314

[38] Peccoralo LA et al. Resident satisfaction with continuity clinic and career choice in general internal medicine. Journal of General Internal Medicine. 2013;28(8): 1020-1027

[39] Robbins MS et al. Procedural headache medicine in neurology residency training: A survey of US program directors. Headache. 2016; 56(1):79-85

[40] Sepulveda D, Varaklis K. Implementing a multifaceted qualityimprovement curriculum in an obstetrics-gynecology resident continuity-clinic setting: A 4-year experience. Journal of Graduate Medical Education. 2012;4(2):237-241

[41] Singman EL et al. Supervision and autonomy of ophthalmology residents in the outpatient clinic in the United States: A survey of ACGME-accredited programs. BMC Medical Education. 2017;17(1):105

[42] Sachs TE, Pawlik TM. See one, do one, and teach none: Resident experience as a teaching assistant. The Journal of Surgical Research. 2015; 195(1):44-51

[43] Philibert I et al. New requirements for resident duty hours. Journal of the American Medical Association. 2002; 288(9):1112-1114

[44] Wojcik BM et al. Structured operative autonomy: An institutional approach to enhancing surgical resident education without impacting patient outcomes. Journal of the American College of Surgeons. 2017;225(6): $713-724 \mathrm{e} 2$

[45] Holzhausen Y et al. Applying occupational and organizational psychology theory to entrustment decision-making about trainees in health care: A conceptual model. Perspectives on Medical Education. 2017;6(2):119-126

[46] Bernstein M, Knifed E. Ethical challenges of in-the-field training: A surgical perspective. Learning Inquiry. 2007;1(3):169-174

[47] Issenberg SB et al. Features and uses of high-fidelity medical simulations that lead to effective learning: A BEME systematic review. Medical Teacher. 2005;27(1):10-28

[48] Sanfey H, Dunnington G. Verification of proficiency: A prerequisite for clinical experience. The Surgical Clinics of North America. 2010;90(3): 559-567

[49] Dijksterhuis MG et al. Assessment of competence and progressive independence in postgraduate clinical training. Medical Education. 2009; 43(12):1156-1165

[50] Teunissen PW et al. How residents learn: Qualitative evidence for the pivotal role of clinical activities. Medical Education. 2007;41(8):763-770 
[51] Wegman B, Stannard JP, Bal BS. Medical liability of the physician in training. Clinical Orthopaedics and Related Research. 2012;470(5): 1379-1385

[52] Ahlberg G et al. Proficiency-based virtual reality training significantly reduces the error rate for residents during their first 10 laparoscopic cholecystectomies. American Journal of Surgery. 2007;193(6):797-804

[53] Williams RG et al. What factors influence attending surgeon decisions about resident autonomy in the operating room? Surgery. 2017;162(6): 1314-1319

[54] Mundschenk MB et al. Are residents prepared for surgical cases? Implications in patient safety and education. Journal of Surgical Education. 2018;75(2): 403-408

[55] Gofton WT et al. The Ottawa surgical competency operating room evaluation (O-SCORE): A tool to assess surgical competence. Academic Medicine. 2012;87(10):1401-1407

[56] George BC et al. Reliability, validity, and feasibility of the Zwisch scale for the assessment of intraoperative performance. Journal of Surgical Education. 2014;71(6):e90-e96

[57] Martin JA et al. Objective structured assessment of technical skill (OSATS) for surgical residents. The British Journal of Surgery. 1997;84(2): 273-278

[58] Mackay S et al. Multiple objective measures of skill (MOMS): A new approach to the assessment of technical ability in surgical trainees. Annals of Surgery. 2003;238(2):291-300

[59] ten Cate O. Nuts and bolts of entrustable professional activities. Journal of Graduate Medical Education. 2013;5(1):157-158
[60] ten Cate O, Scheele F. Competencybased postgraduate training: Can we bridge the gap between theory and clinical practice? Academic Medicine. 2007;82(6):542-547

[61] Farnan JM et al. Strategies for effective on-call supervision for internal medicine residents: The superb/safety model. Journal of Graduate Medical Education. 2010;2(1):46-52

[62] Byrne JM et al. Measuring the intensity of resident supervision in the department of veterans affairs: The resident supervision index. Academic Medicine. 2010;85(7):1171-1181

[63] Kashner TM et al. Measuring progressive independence with the resident supervision index: Empirical approach. Journal of Graduate Medical Education. 2010;2(1):17-30

[64] Kennedy TJ et al. Preserving professional credibility: Grounded theory study of medical trainees' requests for clinical support. British Medical Journal. 2009;338:b128

[65] Kashner TM et al. Measuring progressive independence with the resident supervision index: Theoretical approach. Journal of Graduate Medical Education. 2010;2(1):8-16

[66] DaRosa DA et al. A theory-based model for teaching and assessing residents in the operating room. Journal of Surgical Education. 2013;70(1):24-30

[67] Bohnen JD et al. The feasibility of real-time intraoperative performance assessment with SIMPL (system for improving and measuring procedural learning): Early experience from a multi-institutional trial. Journal of Surgical Education. 2016;73(6):e118e130

[68] Fryer JP et al. Effect of ongoing assessment of resident operative autonomy on the operating room 
environment. Journal of Surgical

Education. 2018;75(2):333-343

[69] Hogle NJ et al. Evaluation of surgical fellows' laparoscopic performance using global operative assessment of laparoscopic skills (GOALS). Surgical Endoscopy. 2014;28(4):1284-1290

[70] Biondi EA et al. Tempering pediatric hospitalist supervision of residents improves admission process efficiency without decreasing quality of care. Journal of Hospital Medicine. 2014;9(2):106-110

[71] Chiong W. Justifying patient risks associated with medical education. Journal of the American Medical Association. 2007;298(9):1046-1048

[72] Feig BA, Hasso AN. ACGME 2011 duty-hour guidelines: Consequences expected by radiology residency directors and chief residents. Journal of the American College of Radiology. 2012;9(11):820-827

[73] Moonesinghe SR et al. Impact of reduction in working hours for doctors in training on postgraduate medical education and patients' outcomes: Systematic review. British Medical Journal. 2011;342:d1580

[74] Greenleaf EK, Aziz F, Hollenbeak CS. Operative autonomy among senior surgical trainees during infrainguinal bypass operations is not associated with worse long-term patient outcomes. Annals of Vascular Surgery. 2017;38: 42-53

[75] Siam B et al. Comparison of appendectomy outcomes between senior general surgeons and general surgery residents. JAMA Surgery. 2017; 152(7):679-685

[76] Shabtai M et al. The impact of a resident's seniority on operative time and length of hospital stay for laparoscopic appendectomy: Outcomes used to measure the resident's

laparoscopic skills. Surgical Endoscopy. 2004;18(9):1328-1330

\section{[77] Wong K, Duncan T, Pearson A.}

Unsupervised laparoscopic

appendicectomy by surgical trainees is safe and time-effective. Asian Journal of Surgery. 2007;30(3):161-166

[78] Fahrner R, Schob O. Laparoscopic appendectomy as a teaching procedure: Experiences with 1,197 patients in a community hospital. Surgery Today. 2012;42(12):1165-1169

[79] Graat LJ et al. Appendectomy by residents is safe and not associated with a higher incidence of complications: A retrospective cohort study. Annals of Surgery. 2012;255(4):715-719

[80] Day KM et al. Progressive surgical autonomy in a plastic surgery resident clinic. Plastic and Reconstructive Surgery. Global Open. 2017;5(5):e1318

[81] Brandel MG et al. Analysis of a resident aesthetic clinic: Process for rhinoplasty, resident experience, and patient satisfaction. Annals of Plastic Surgery. 2017;78(5 Supp. 4): S175-S179

[82] Walker NJ et al. Outcomes analysis of chief cosmetic clinic over 13 years. Annals of Plastic Surgery. 2018;80(6): 600-606

[83] Kantor O et al. Implementing a resident acute care surgery service: Improving resident education and patient care. Surgery. 2017;161(3):876-883

[84] Wojcik BM et al. The resident-run minor surgery clinic: A pilot study to safely increase operative autonomy. Journal of Surgical Education. 2016; 73(6):e142-e149

[85] Jarman BT et al. Enhancing confidence in graduating general 
surgery residents: Establishing a chief surgery resident service at an Independent Academic Medical Center. Journal of Surgical Education. 2018; 75(4):888-894

[86] Kairys JC et al. Cumulative operative experience is decreasing during general surgery residency: A worrisome trend for surgical trainees? Journal of the American College of Surgeons. 2008;206(5):804-811; discussion 811-3

[87] McLaren K et al. Ownership of patient care: A behavioural definition and stepwise approach to diagnosing problems in trainees. Perspectives on Medical Education. 2013;2(2):72-86

[88] Chen XP et al. Assessing residents' readiness for OR autonomy: $A$ qualitative descriptive study of expert surgical teachers' best practices. Journal of Surgical Education. 2017;74(6): e15-e21

[89] Beck J et al. Promoting resident autonomy during family-centered rounds: A qualitative study of resident, hospitalist, and subspecialty physicians. Journal of Graduate Medical Education. 2016;8(5):731-738 



\title{
Teaching Balanced Patient Care Using Principles of Reductionism and Holism: The Example of Chronic Low Back Pain
}

\author{
Alan Remde, Stephen DeTurk and Thomas Wojda
}

\begin{abstract}
This chapter explores how integrating holistic and reductionistic approaches to care may better optimize value based care. First, we define the terms 'Holistic', 'Reductionistic' and 'Integrative'. Then we explore their scope in the arenas of teaching and patient care, with the advantages, disadvantages and pitfalls of each approach. We review how these styles are embedded in and interact with the cultures of medicine and western societies at large. As an example of a balanced care approach, we focus on the example of chronic low back pain (CLBP), an increasingly common and expensive medical problem. We present practical examples of teaching and practicing these different styles, Holism and Reductionism, illustrating when each may be appropriate to optimize value of patient care. Study questions are included. A list of further readings and resources is included for the interested reader.
\end{abstract}

Keywords: graduate medical education (GME), holism, reductionism, chronic pain, chronic low back pain (CLBP), value based care

\section{Introduction}

Present U.S. graduate medical education (GME) teaches knowledge, skills and attitudes to future providers in a health care system that spends a higher \% of GDP with often poorer outcomes for chronic disease care than most other industrialized nations $[1,2]$. Much of the chronic disease burden is driven by preventable risk factors such as poor diet and exercise patterns and risky behaviors such as substance abuse $[3,4]$. This unsustainability is driving rapid transition to value based care, with payers demanding better outcomes for their dollars spent. Given the unsustainability and suboptimal effectiveness of our present chronic disease care, we might ask how GME needs to change in order to deliver more effective, value based care. This chapter explores how integrating holistic and reductionistic approaches to care may better optimize value based care, using as an example the dilemmas surrounding treatment of chronic low back pain (CLBP). Such integrative care may also lead to better physician satisfaction and less burn-out, as discussed later in this chapter. 


\section{Definition and descriptions of terms used in this chapter as related to healing and medical care}

\subsection{Reductionism}

Reductionism: the practice of analyzing complex phenomenon in terms of its fundamental constituents, with the intent to manipulate these constituents to improve the whole or health. Reductionism is a style of perception, teaching and practicing medicine that focuses on addressing symptoms and proximate (and therefore more easily measurable) causes.

1. An implicit assumption of reductionism is that correcting the part will enhance wholeness. That is, that we have enough understanding of how the part relates to the whole that we can safely and effectively manipulate it to improve wholeness or health.

2. Implied in the practical application of reductionism (e.g. the use of many pharmaceuticals) is the assumption that treating symptoms of a disease is a reasonable surrogate to healing the whole person.

3. Concepts and fields of inquiry that are emphasized in a reductionistic approach to medicine focus on analysis of body structure and function, such as anatomy and physiology, and methods of manipulating these, such as with surgery or pharmaceuticals.

4. Evaluating and treating a 'part' can have many advantages. It is often more practical, expedient and parsimonious in its use of health care resources. For example, an inflamed appendix is diagnosed as the proximate cause of acute abdominal pain with a CT scan and surgically removed, or a fractured hip is diagnosed by X-ray as the proximate cause of acute hip pain and pinned. Most would agree that this 'fix it' approach makes eminent sense in these acute cases and leads to a better health outcome for these patients.

5. Our healthcare system is largely built on this reductionistic paradigm. In this paradigm, focus is on disease treatment, not wellness. The characteristic of the patient that has the disease is much less important. For example, diabetes mellitus type 2 (DM2) or chronic low back pain (CLBP) is treated more or less the same regardless of which patient has it. Likewise, 'providers' are largely interchangeable, since care algorithms are tailored to the disease rather than the patient or the patient-physician relationship. The health of the relationship between a patient and their physician is given much less importance. In these and other chronic diseases, the same approach used in acute care of treating the simple proximate cause is often taken. For example, an easily measured proximate cause of microvascular complications in DM2 is hyperglycemia, so focus is often on treating this with pharmaceuticals that lower blood sugar. While certainly worthwhile as part of a therapeutic strategy, when hyperglycemia treatment is done to the exclusion of a large number of other issues in DM2, sub-optimal outcomes may result. Examples of less easily measurable issues in DM2 include addressing habituation to highly processed 'pseudo-food' such as snacks that have manipulated fat, salt \& sugar content [5], correcting the body's gut microbiome (which is altered in DM2 [6], improving vascular compliance and possibly autonomic balance $[7,8]$, calming chronic inflammation, reversing 
hepatic metabolic derangements related to steatosis, improving insulin sensitivity [9], decreasing excess load of advanced glycation end products [10,11] and many other less easily measurable but important aspects of DM2 treatment. Less attention to these other important factors may be one reason that present DM2 treatment algorithms have suboptimal outcomes, for example with macrovascular disease. Advice to eat right and exercise is commonly given, but very little resources or time is devoted to helping the patient actually accomplish these profound lifestyle changes in most DM2 care algorithms. Most doctors have very little training in nutrition or exercise science and thus may feel less qualified to guide the patient in these areas. A care model focusing on individual office visits is unlikely to allow delivery of the depth of lifestyle guidance actually required for significant change. If quality of care is narrowly defined by if glycemic goals are met, providers will focus on meeting this metric.

6. Many providers and patients perceive the overall approach of our present healthcare system as problematic, expressing concerns about 'hamster wheel medicine'-attempts to treat complex issues in 15 minute slots, over focus on treating symptoms rather than underlying cause, dissatisfied patients, epidemic levels of provider burn-out and soaring health expenditures as unintended consequences of our present Healthcare model.

7. The development of reductionist approaches to medical care, with its underpinning of largely reductionistic scientific methods, has led to enormous advances. This is especially true in the areas of acute care, such as emergency medicine, critical care medicine and surgery. However, serious limitations become apparent when this same reductionistic paradigm is applied to chronic disease. The major modifiable factors driving chronic diseases, such as obesity, diabetes mellitus type 2, chronic obstructive pulmonary disease (COPD), heart disease, depression and chronic pain are related to lifestyle and/or environmental influences. Outcome studies in general show sub-optimal results when reductionistic methods, such as an over-focus on pharmaceuticals are applied to many chronic diseases. Our present reductionistic models seem impotent when faced with the rising tide of obesity, DM, depression and chronic pain. A new paradigm is needed.

\subsection{Holism}

Holism: a style of perception, teaching and practice of medicine based on the axiom that the whole is irreducible and cannot be adequately understood by simply understanding the parts. MeSH Scope defines "Holistic Health" as "Health as viewed from the perspective that humans function as complete, integrated units rather than as aggregates of separate parts."

1. The implicit assumption of a holistic approach to care is that enhancing wholeness will lead to healing of any dysfunctional parts that are manifesting as disorder or disease. This also assumes that it is within the power of the patient (with the facilitation of the healer) to enhance this restoration toward wholeness.

2. 'Wholeness' implies balance and integrity within the patient and in their relationships. For example, a person is more 'whole' if they not only have less disease symptoms, but also have maintained a sense of internal emotional integrity, life meaning, and family and social relationships. 
3. The focus of holistic approaches to patient care are on overall wellness as relates to the presenting disorder of the patient, including healthy lifestyle aspects such as healthy eating, movement (exercise), rest \& sleep, personal and social relationships and relationships to a higher power or intelligence.

4. Concepts and fields of inquiry that are emphasized in holistic healing include whole food plant-based approaches to eating, meditative movement to support mind/body harmony and insightful emotional processing leading to emotional/energy balance, supportive relationships, including at inter-personal, family and community levels, cultural and socioeconomic justice, environmental health and spiritual relationship.

5. The assumption that 'healing the whole will heal the part' implies placing the locus of control more on the patient than the physician. The healer is more of a 'facilitator' rather than someone who 'fixes' the patient. This fundamentally changes and makes paramount the relationship between physician and patient, and places more importance on adequate time for this relationship to develop and be maintained. It also implies that the characteristics of the patient that has the disease are equally or more important than the particulars of the disease the patient has.

6. Skill sets emphasized in holistic care include the following:
a. intuitive and emotional perception and processing
b.developing skills in listening, understanding and communication
c. ability to sense and find balance and harmony

\subsection{Integrative care}

Integrative care for the purpose of this chapter means an approach that attempts to balance reductionistic and holistic approaches to medical care and healing to

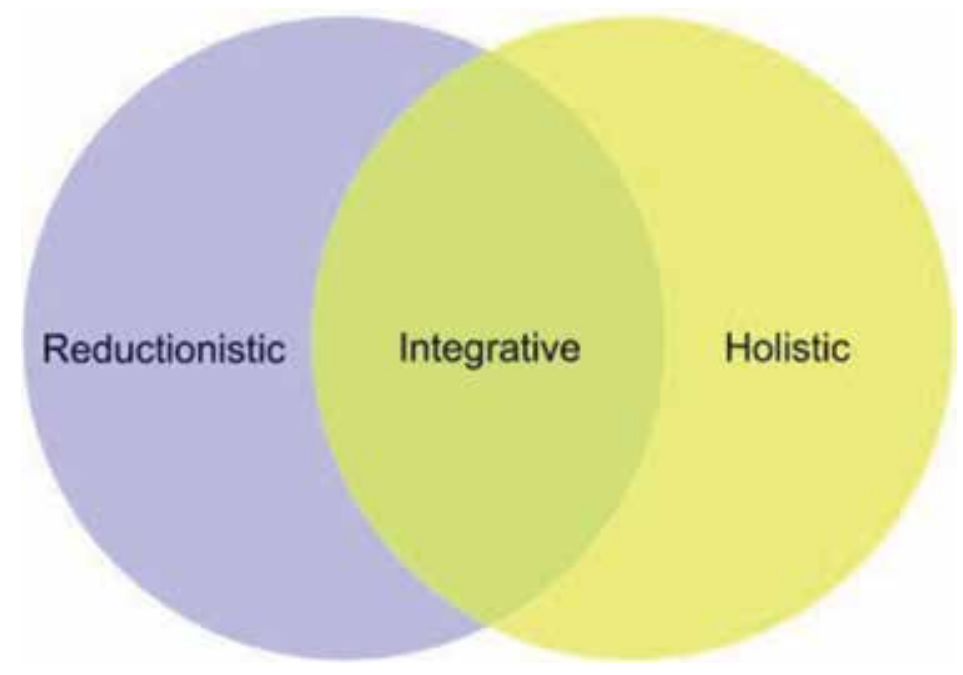

Figure 1.

Integrative care is the overlap between holistic and reductionistic methods. 
optimize patient outcomes. Concepts and fields of inquiry that are emphasized in integrative care include complexity and systems theory, mind/body integration related fields such as psychoneuroimmunology and an expanded biopsychosocial model that emphasizes supporting a healthy lifestyle in all its aspects. An integrative approach to care may be beneficial not only for patients but also for the physician. Integrative approaches support in depth, meaningful relationships between physician and patient and adequate time to develop these relationships, which may reduce burn-out [12] (Figure1).

\section{Balancing reductionistic with holistic aspects of care}

Metaphorically, reductionism is akin to viewing electromagnetic energy such as light as a particle, holism to viewing light as a wave. Each apparently contradictory viewpoint may have validity and usefulness in different contexts. Choosing the right approach based on the needs of the situation is the goal of an integrative approach in order to optimize value based patient care. All systems of care have holistic and reductionistic aspects, though one aspect may be more emphasized. The biopsychosocial model used in primary care in Westernized medicine is more on the holistic side of the spectrum, while procedural and surgical techniques are more on the reductionistic side. Compared to many indigenous healing systems such as Traditional Chinese and Ayurvedic medicine however, Western conventional medicine tends toward a more reductionistic approach. We may be going thru a stage of necessary re-balancing, with more emphasis on holistic aspects such as lifestyle medicine. However, this attempt to combine these two approaches presents its own set of new challenges.

\section{Common misperceptions, difficulties and pitfalls in conventional and holistic medicine}

\subsection{Cautions in initial approaches to combining healing systems}

\subsubsection{Simplistic, categorical descriptions of healing systems as 'reductionistic' or 'holistic' should be avoided}

All healing systems have reductionistic and holistic aspects. The practice of prescribing many supplements by some 'alternative medicine' practitioners is highly reductionistic, while a 'conventional' medical doctor empathically listening to a patient's illness story is more holistic.

\subsubsection{Categorizing conventional medicine as 'scientific', while less familiar healing systems as 'unscientific' is not helpful}

This is often used to imply that only Western conventional medicine has 'validity'. All healing systems are embedded in and reflect the culture they exist in, with all that culture's strengths, limitations, blind spots and implicit assumptions. Evidence based medicine teaches us that only about $18 \%$ of conventional primary care medical practice has strong scientific support [13]. Other healing systems also have a mix of scientific and more empirical support. Thus it is not reasonable to assert a simplistic dichotomy of conventional medicine as 'scientific' and other healing systems as 'non-scientific' [14]. 


\subsubsection{Assuming one can take a healing modality out of context of the original healing system it was used in and have similar efficacy can be problematic}

An example of taking a healing modality out of context is acupuncture. While acupuncture may be useful as a 'stand alone modality', it is quite possible that its effects in the larger context of Traditional Chinese medicine where it originated may be quite different.

\subsection{Pitfalls in research}

\subsubsection{Conflating limitations in scientific methodology for lack of validity of holistic systems}

While outcome studies can assess a holistic care modality in its entirety, there are severe limitations in our present scientific methodology needed to understand whole systems in general. This lack of scientific methodology does not necessarily mean lack of validity, anymore than a lack of perspective of a person on the face of the earth means the earth is flat rather than spherical.

Conversely, the lack of a deep theoretical framework to understand holistic systems also means that their efficacy should not just be assumed. All holistic systems of care need extensive outcome studies to demonstrate efficacy.

Use of sham therapy in studies for holistic treatments (e.g. osteopathic manipulation, acupuncture, yoga) does not take into consideration the possible benefits of sham therapy, and studies showing equivocal outcomes may falsely imply the benefits of these treatments are due to placebo effects $[15,16]$.

\subsubsection{Confusing the immeasurable with the invalid}

Present research techniques emphasize quantitative approaches of what is scientifically easily measurable. While this is practical and important, it does not imply that qualitative, experiential and other approaches assessing intangible factors are less valid. Over-reliance on quantitative approaches to research is itself a distorted lens of perceiving reality. Over-reliance on measurable parameters while discounting intangible factors in healing can lead to a 'tyranny of the measurable'.

Relationship between therapist and client appear to be one of the most important aspects of outcome in psychotherapy, rather than the training or type of therapy used, even though these are more measurable than the intangibles of relationship.

\subsubsection{Making conclusions on whole systems based on highly reductionistic scientific studies of a part of these systems}

Scientific studies of single nutrients are often not relevant to the effects of whole foods. For example, studies on the effects of megadoses of synthetic vitamin $\mathrm{E}$ do little to inform us of the effects of whole foods that contain vitamin $\mathrm{E}$ [17]. Similarly, nutrients can have widely varying and sometimes opposite effects depending on whether the research subject is deficient or not in the nutrient. Treating nutrients as if they are pharmaceuticals, either in research or in clinical practice, often leads to spurious results and confusion.

\subsubsection{Assuming scientific studies on efficacy or side effects in an acute care setting apply to a chronic disease care setting}

Studies showing efficacy of opioids in acute pain were assumed to apply to chronic pain, a very different situation, with sometimes disastrous results including 
Teaching Balanced Patient Care Using Principles of Reductionism and Holism: The Example... DOI: http://dx.doi.org/10.5772/intechopen.82618

contributing to the opioid epidemic $[18,19]$. Similarly, the multiple side effects and disadvantages to chronic proton pump inhibitors use were not appreciated or understood by studies demonstrating their short term efficacy.

\subsubsection{Basing conclusions on studies limited in scope to one culture}

Cross cultural nutrition studies show much more significant and often dramatic effects of dietary patterns on chronic disease compared to comparisons of dietary patterns confined to Western societies, because the variation in eating styles in other cultures are sometimes much wider than in Western cultures. Confining nutrition research to Western eating habits can lead to a myopic view [11].

\subsection{Approach to clinical care}

\subsubsection{Confusing surrogate biomarkers for the disorder, and hence mistaking correction of the surrogate biomarker for healing}

High blood pressure is an important index measure but only one aspect of hypertension, whose pathophysiology includes decreased arterial compliance, altered sympathetic - parasympathetic balance and other pathophysiological issues. Lowering blood pressure, while important per se, is only a partial correction of the pathophysiology of hypertension [20]. While lifestyle issues such as healthy diet, stress management and rest are usually mentioned, they are often given less than necessary attention. Similarly, as stated previously, hyperglycemia is an important index measure but not equivalent to diabetes. An overly pharmaceutical centered approach does little to address the underlying metabolic derangements of DM2.

\subsubsection{Assuming symptom suppression is equivalent to healing}

Proton pump inhibitors (PPIs) suppress acidity of gastric reflux, but do little to reduce the reflux itself or to address its underlying causes, which is often related to poor diet or eating habits, and expose the patient to a number of increase risks [21-24].

\subsubsection{Underestimating the resources needed for holistic healing modalities}

Many lifestyle changes and holistic care modalities require the patient to invest extensive time, attention and sustained effort. Unrealistic expectations of quick change are unfair and demoralizing for the patient and a set-up for failure. For example, changing eating habits likely requires extensive education, social support and reinforcement over many months. It is unrealistic to just tell a patient to 'eat more healthy' and expect much change.

\subsubsection{Assuming that supplements, botanical medicines or natural modalities are safer and superior (or more dangerous and inferior) to pharmaceuticals}

Such wide generalizations are neither prudent nor supported by evidence. Practitioners tend to view what they are familiar with as safer and more efficacious than less familiar approaches. Thus, conventional medicine oriented practitioners may categorize natural medicines as inferior and 'unscientific', while holistic oriented practitioners may assume that natural medicines must be better than pharmaceuticals. While many common natural supplements and botanicals appear relatively safe, there is no intrinsic reason that they should be worse or better than pharmaceuticals. For example, anti-HIV medications have no equivalent among botanicals and 
are clearly life-saving. Gut-directed hypnotherapy and enteric coated peppermint oil are efficacious and safe for certain irritable bowel syndrome symptoms [25, 26]. NSAIDs when used chronically have significant and often under-appreciated toxicity. Some commonly used botanicals can be hazardous if not formulated correctly. Kava kava, for example, should only be used in aqueous formulations, since alcohol extracts may lead to liver toxicity [27]. Similarly, while many botanicals can be used safely with many pharmaceuticals, on occasion clinically significant interactions can occur between them. It behooves the practitioner to be aware of these interactions (can access standard references such as Natural Medicines Database) or to consult a qualified integrative medicine practitioner when needed.

\section{Teaching balanced care using principles of reductionism and holism: the example of chronic low back pain (CLBP)}

\subsection{Chronic pain background}

Chronic pain, and in particular chronic low back pain (non-cancer related) is chosen to illustrate an integrative model described herein because of its large burden of suffering and difficulties in treatment with present models of care. Chronic pain in general has an enormous influence on the emotional, physical and social function of patients and society at large and effects about 100 million people in the US alone [28, 29].

\subsubsection{Approaches to CLBP}

CLBP has a wide range of causes that may benefit from different approaches. Some cases appear caused by specific anatomic derangements such as a herniated disc pressing on a nerve root-the 'nociceptive generator', which lend themselves to a 'fix it' reductionistic approach such as epidural injections or surgery. More general approaches such as physical therapy to build core strength and control can be helpful in many cases of CLBP. Muscle tightness per se can be a nociceptive generator and will often benefit from mind/body and physical therapy approaches. However, in many cases, a specific lesion is not identifiable, and there appear to be significant psychosocial factors related to how the patient experiences pain. Statistically, the biggest predictor of chronic pain, including CLBP, is the 'emotional charge' surrounding the disorder-mood, meaning of the pain in the patient's life, psychosocial consequences. Neuro-anatomic imaging of the spine (such as MRI) has variable correlation to severity and duration of chronic low back pain [30, 31]. The challenge for the GME teacher is to teach ability to discern these widely varying etiologic factors and to apply the most effective approach in each case. The efficacy of a sample of widely varying approaches is illustrated below.

\subsubsection{Challenges}

Chronic pain including CLBP is a highly subjective experience influenced by biological, psychological, and social factors. Alterations in central and peripheral nervous system processes add to its complexity and challenge [32]. Sleep disturbance, anxiety, and depression often accompany chronic pain [33]. These in turn may exacerbate the pain and further reduce quality of life [34]. For example, anxiety and sleep disturbance each occur in about half of people with chronic pain [35]. Depression has been linked with an increase in pain severity in $21-72 \%$ of cases [36]. The challenges of chronic pain are exemplified by returning war veterans from tours in Iraq and Afghanistan. Almost half have chronic pain [37], most which 
is musculoskeletal related [38]. Prevalent among military veterans is their high incidence of comorbid post-traumatic stress disorder (PTSD) and traumatic brain injury (TBI), which likely aggravate their chronic pain syndromes [39].

\subsection{Reductionist approaches}

Reductionist treatments include pharmaceuticals and interventional treatments. Pharmacological treatment of chronic back pain does not correct the underlying cause of back pain, but instead focuses on reduction of pain allowing improvement of functionality. Interventional treatment includes nerve blocks, injections at various points (e.g. trigger point, epidural and facet joint injections), nerve ablations, or surgery. The efficacy of these various treatments is highly dependent on the etiology of the pain.

\subsubsection{Pharmaceuticals}

Pharmaceutical treatments for CLBP provide relief of symptoms, decreasing pain and improving function. However, pharmaceutical interventions in isolation do not address the origin of pain. Correcting the pathology causing pain is better accomplished with exercise, physical therapy, or spinal manipulation. Pharmaceuticals provide a supporting role in helping patients tolerate pain that may result from some of these treatments. Studies show that medication plus exercise based therapy, performs better than either in isolation [40].

Additional consideration must be given to possible side effects when prescribing medications. NSAIDs are the first line pharmaceutical treatment recommended, while tramadol and duloxetine are second line treatment options [40]. The use of opiates for treating chronic non-cancer pain including CLBP is highly problematic, with strong evidence of harm and growing evidence they are no better than nonopioid analgesics for many chronic pain syndromes, including CLBP [19].

\subsubsection{Interventional}

In cases where the etiology of back pain can be determined, treatments can be specifically targeted. Radiofrequency ablation targets sources of pain at specific anatomic sites, as determined by a diagnostic nerve block. Systematic reviews of the technique showed significant reduction in back pain over an extended period in some cases [41], however a large RCT showed radiofrequency ablation combined with exercise provided no benefit over exercise alone [42].

Surgical discectomy is superior to conservative management in the treatment of lumbar disc prolapse [43], however this treatment would not be beneficial in cases of lumbar strain. A less obvious difference in treatment outcomes can be seen with transforaminal epidural steroid injections. A review article found good evidence to support transforaminal epidural injections (steroid + local anesthetic) in cases of radiculitis secondary to disc herniation, but there was insufficient evidence in cases of less specific axial pain and post surgery syndrome [44].

\subsection{Holistic approaches}

Even in patients who have not suffered war or other physical trauma, trying emotional experiences such as interpersonal conflicts, work stress, and social rejection can strongly influence the experience of chronic pain $[45,46]$. These include unresolved struggles with family members, conflicts over perfectionism, stigmatized desires that lead to shame, ambivalence toward one's children, and various other drivers of stress reactions. The main pathological process in both unresolved 
emotional trauma and internal conflict may be the evasion or concealment of emotions, which are believed to activate neural pathways that augment pain and other symptoms. Focusing on resolving these unprocessed emotions related to unresolved emotional trauma, conflict, and relational disturbances often improves chronic pain. This applies to a wide range of chronic pain syndromes including musculoskeletal pain such as CLBP, fibromyalgia, headaches, irritable bowel syndrome, and chronic pelvic pain [46-49]. Figure 2 illustrates one possible model to address these issues.

\subsubsection{Mind-body integration}

In cases of nonspecific back pain, it is not possible to identify a specific nociceptive generator leading to pain. In these situations, a holistic treatment approach to the whole person instead of a lesion may be preferable. Studies have shown various holistic treatments to provide significant outcome. The MORE study evaluated the effect of mindfulness training, cognitive reappraisal, and positive emotion regulatory strategies as a combined treatment in patients with chronic pain who were taking opiates. Early results showed the combination treatment group had lower pain severity, less functional interference, and less desire for opioids compared to the standard support group treatment [51].

\subsubsection{Exercise/rehabilitation}

Exercise and yoga have been proven to be effective treatments for CLBP. GME should not only focus on teaching about the EBM supporting these treatments, but also teach about regional resources that are available to patients.

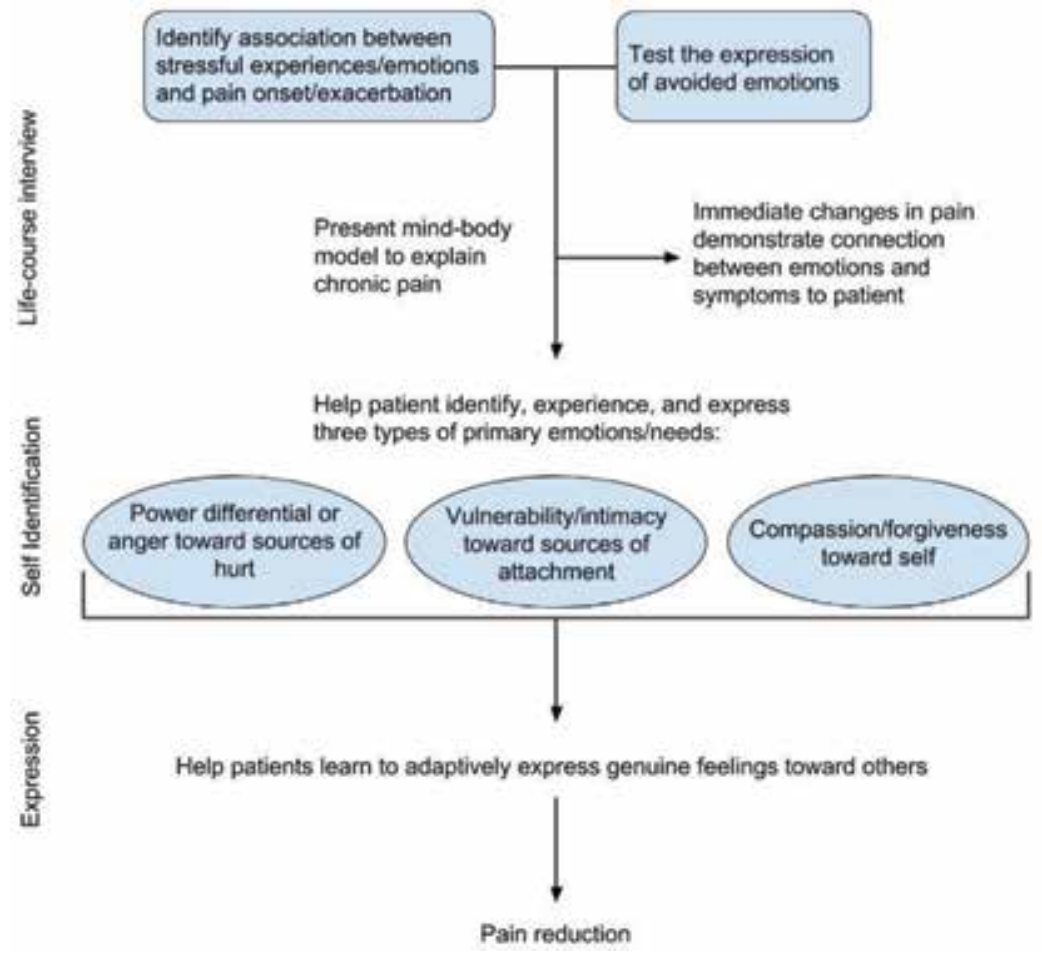

Figure 2.

A sample of intervention technique focused on the mind-body model [50]. This model explains how chronic pain due to neural pathways formed by prior maladaptive learning and maintained by emotional avoidance can be "unlearned" with powerful corrective emotional experiences. 
Teaching Balanced Patient Care Using Principles of Reductionism and Holism: The Example... DOI: http://dx.doi.org/10.5772/intechopen.82618

A systematic review examined a holistic treatment approach to chronic back pain. Multidisciplinary biopsychosocial rehabilitation (MBR) involves a combination of physical, psychological, and educational treatments usually provided by a multidisciplinary care team for the treatment of chronic back pain. The review found that MBR had moderate improvements in short and long term (1-2 years) pain intensity as well as disability compared to usual care. MBR also outperformed physical interventions (e.g. physiotherapy, exercise) in short term pain reduction, as well as short- and long-term disability. Patients receiving MBR were also twice as likely to be working after 1 year compared to physical interventions [52]. Integrating MBR into GME could be easily accomplished by having residents serve as part of the care team for select patients with uncontrolled back pain.

\section{Chronic Pain evaluation and treatment algorithm}
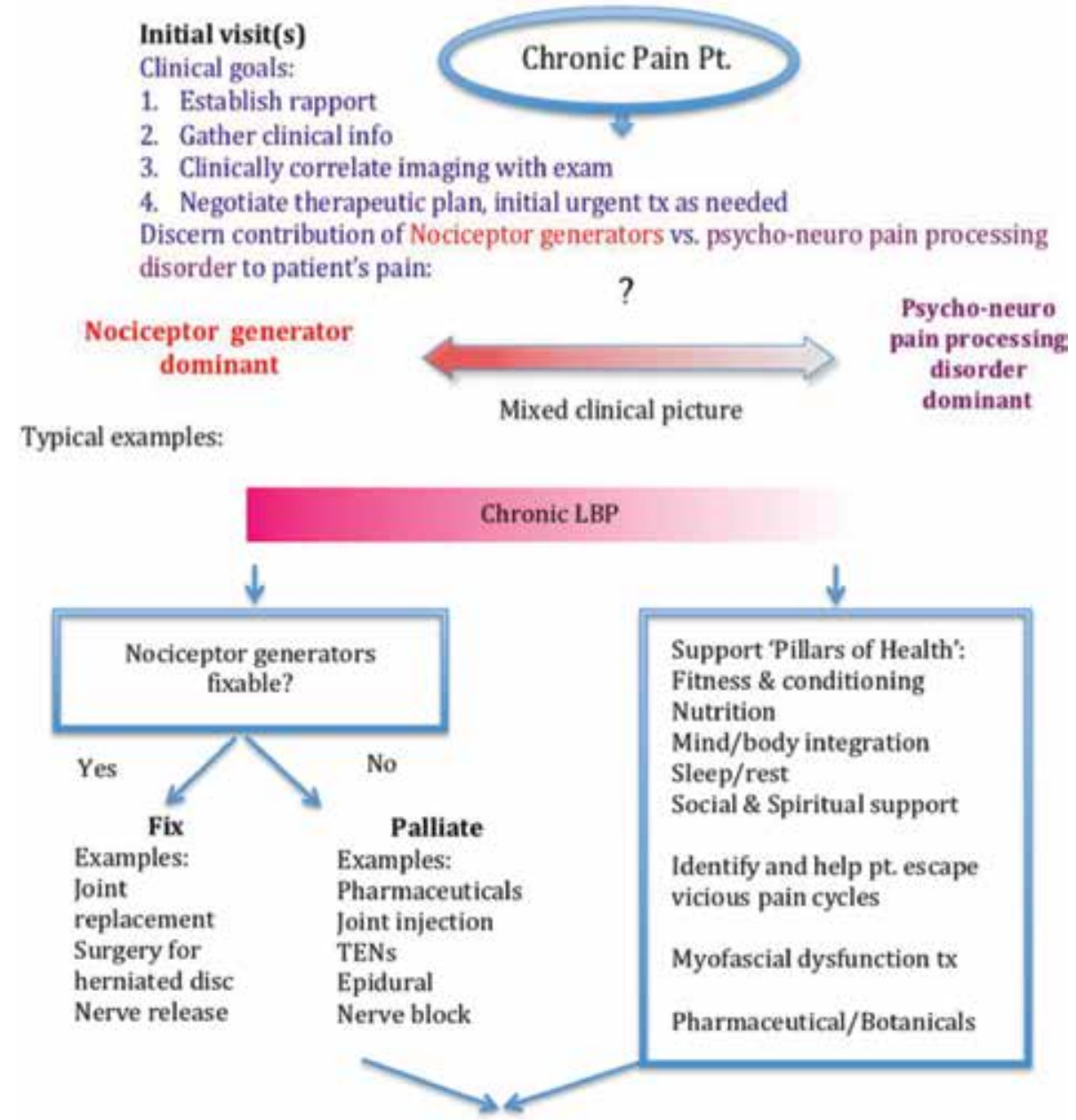

Maintenance visits (e.g. 30 minutes q month) Clinical goals:

1. Maintain therapeutic alliance

2. Monitor and adjust therapeutic plan

3. Set \& monitor pain and verifiable functional goals

Figure 3.

Sample schematic on approach to chronic pain and in particular CLBP that integrates both reductionistic 'fix it' and more holistic general support approaches. 


\subsubsection{Energy medicine treatments}

Acupuncture is recommended as a first line treatment option for CLBP [40]; however its basis on an unfamiliar Eastern paradigm involving energy meridians may cause some skepticism among conventional medicine doctors. Research shows that acupuncture therapy produces measurable physiologic effects [53], and randomized controlled trials demonstrate moderate improvement in function and pain reduction. Comparisons to sham acupuncture have had mixed results, but meta-analyses show small improvement. A Cochrane review of 35 RCTs of moderate quality, showed moderate lower pain $\&$ improved function vs. no acupuncture. When comparing acupuncture to other treatment modalities, there is again some inconsistency. Low quality studies showed small improvement in pain and function compared to various medications such as NSAIDs. When combined with usual care however, acupuncture and usual care outperformed usual care alone [54]. A sample schematic integrating reductionistic and holistic approaches to CLBP is noted in (Figure 3).

\section{Teaching competencies}

Given the widely variant causes of chronic pain, including CLBP, (as well as the causes of many other chronic disease entities), the next question is, how would the GME teacher approach teaching competencies in such a wide range of skill sets? Literature searches on the topic identified articles about teaching holism and wellness to medical students for the purpose of self care. However, there was sparse literature available focused on GME approaches to teaching application of general holistic care for treating patients.

Based on the author's experience, a few examples of general principles in GME teaching of reductionistic and holistic approaches to care of chronic disease are offered below. Some of these are obvious, others not so much:

\subsection{Teach basic precepts of reductionistic approaches}

Thorough and precise theoretical framework of body structure and function, and knowledge of and identification of specific disorders is key to successful specific treatments. Any interventional procedure skill requires ample well supervised hands on practice.

\subsection{Teach basic precepts of holistic approaches}

Teach a theoretical framework of mind/body unity, including evidence base of such fields as psychoneuroimmunology and meditative movement therapies.

Self-regulation of affect and soma can lead to less pain and better function. Skill sets that support this process include:

1. Mindful awareness of and resolution of intra- and inter-personal unconscious and conflictual emotional processes (as exemplified in the above mind/body model)

2. Development of 'kinesthetic intelligence'-awareness and interpretation of the body's 'language' of movement patterns and holding neuromuscular tension and postures. Understand how this correlates with emotional states and musculoskeletal pain syndromes. This also requires 'hands on' experiential 
practice, for example thru lessons in one of the mind/body practices such as Tai chi, Yoga, Feldenkrais or Somatics.

We suggest that much work is needed in GME education of both the reductionistic as well as the holistic skill sets listed above. Identification of teachers who can guide learners in these skill sets acquisition is important.

Any interventional procedure skill requires ample well supervised hands on practice.

\subsection{Approaches and modalities in caring for CLBP patients (and patients in general of course) should be guided mainly by the needs of the patient}

A common tendency for us providers is to perceive the patient's disorder and needs thru the lens of our own experience and professional scope of knowledge. Hence for the same CLBP patient the primary care physician may prescribe an NSAID and back stretches, the Naturopath may include a botanical such as curcumin, the interventionalist an epidural, the chiropractic manipulation. It is obvious that this is a suboptimal approach. Ideally, a generalist with a wide range of familiarity with different reductionistic and holistic modalities would first perform a careful evaluation of the patient's individual situation. They would then develop a reasonable assessment of the causes of their symptoms, including mechanical, emotional and spiritual factors. Then they would use carefully selected tests to support or refute their clinical hypothesis. They would take into account the personality and resources of the patient themselves. Then they would synthesize all these findings into a coherent therapeutic plan, making sure the patient understands and agrees with it and can reasonably carry it out. They would refer to other providers as needed, gathering a team to help the patient. Referrals could include reputable practitioners in both more reductionistic fields such as interventional pain and surgery and more holistic oriented fields such as physical therapy, psychotherapy, acupuncturists and Tai Chi teachers. Often such a team approach is key, since very few of us have mastered the wide range of skill sets that might benefit a patient.

\subsection{In specialties such as primary care (e.g. family medicine, internal medicine, pediatrics) in which a wide range of skill sets is expected, residencies need to identify teachers who can teach these skill sets}

For example, curriculum should include at least familiarity with holistic approaches such as mind/body medicine and acupuncture in their training. Ideally, there should be training in basic holistic techniques that can be done by the primary care physician such as stress management techniques and select natural medicine that have a good evidence base of effectiveness. Learning how to properly refer to holistic oriented practitioners such as Tai Chi, Yoga teachers and acupuncturists is important (Table 1).

6.5 Primary care residencies should also teach clear indications and evidence base for interventions and other reductionistic techniques

6.6 In more narrow focused specialties such as orthopedic surgery, residents should at least be exposed to the evidence base for holistic modalities. Teach and assess competency on choosing which patients would benefit from these modalities and how to appropriately refer

\subsection{Reductionist and holistic CLBP competency examples}




\begin{tabular}{|c|c|c|}
\hline $\begin{array}{l}\text { ACGME core } \\
\text { competency }\end{array}$ & Reductionistic approaches & Holistic approaches \\
\hline Patient care & $\begin{array}{l}\text { Discerning and correlating exam/imaging } \\
\text { findings to decide the contribution of } \\
\text { neuro-anatomical derangement vs. pain- } \\
\text { processing disorder } \\
\text { Effective pain suppression with } \\
\text { pharmaceuticals while optimizing risk/ } \\
\text { benefit } \\
\text { Effective referrals, e.g. to PT, Pain } \\
\text { specialists, proceduralists (e.g. for Epidural) }\end{array}$ & $\begin{array}{l}\text { Guiding patients in mind/body } \\
\text { awareness and processing of } \\
\text { nociceptive signals, spiritual } \\
\text { re-connection and meaning of pain } \\
\text { experience } \\
\text { Use of simple stress management and } \\
\text { psycho-therapeutic techniques } \\
\text { EBM supported botanicals and } \\
\text { understanding of possible botanical- } \\
\text { pharmaceutical interactions }\end{array}$ \\
\hline $\begin{array}{l}\text { Medical } \\
\text { knowledge }\end{array}$ & $\begin{array}{l}\text { Neuroanatomy of spine and spine lesions } \\
\text { Referral indications for interventionist } \\
\text { approaches such as Epidurals, neurosurgery }\end{array}$ & $\begin{array}{l}\text { Familiarity and basic applications } \\
\text { of Functional movement practices, } \\
\text { Whole foods anti-inflammatory diet, } \\
\text { Energy medicine approaches such as } \\
\text { acupuncture }\end{array}$ \\
\hline $\begin{array}{l}\text { Practice-based } \\
\text { learning \& } \\
\text { improvement }\end{array}$ & $\begin{array}{l}\text { QI of CLBP patients Appropriateness } \\
\text { criteria for spine imaging }\end{array}$ & $\begin{array}{l}\text { QI of CLBP patients effectiveness of } \\
\text { meditative movement referrals (e.g. } \\
\text { Yoga, Somatics) }\end{array}$ \\
\hline $\begin{array}{l}\text { Interpersonal \& } \\
\text { communication } \\
\text { skills }\end{array}$ & $\begin{array}{l}\text { Effective communication of therapeutic } \\
\text { assessment \& plan }\end{array}$ & $\begin{array}{l}\text { Ability to facilitate mindfulness and } \\
\text { release of blocked emotions and } \\
\text { related muscle tension }\end{array}$ \\
\hline Professionalism & Pt. survey scores on professionalism of care & Similar \\
\hline $\begin{array}{l}\text { Systems-based } \\
\text { practice }\end{array}$ & $\begin{array}{l}\text { Learner demonstrates effective referral to } \\
\text { pain specialists }\end{array}$ & $\begin{array}{l}\text { Learner demonstrates effective referral } \\
\text { to holistic practitioners }\end{array}$ \\
\hline
\end{tabular}

Table 1.

Example competencies for treating CLBP in primary care residency.

\section{Examples of chronic low back pain (CLBP) patients}

\subsection{Example 1}

Mr. Smith is a 45 y/o man with CLBP ranging in severity from 3 to $8 / 10$ for 7 months that began after a warehouse work accident in which he herniated his right L4/5 disc with sciatic symptoms down his right leg. His symptoms are aggravated by his job where he needs to lift boxes and so he was put on temporary disability. His clinical and MRI exam correlate well. He is otherwise healthy and well adjusted with no comorbidities. Physical therapy (PT) was only modestly helpful.

Study questions:

1. Would you focus on a more reductionistic 'fix it' approach or a more holistic approach in this case? Why?

2. What would be your next steps?

3. What pharmaceutical classes might be most useful for his pain?

4. What procedures might be most useful in this case?

5. Name 3 competencies that would be most relevant for learners in this case?

6. How would you approach teaching these 3 competencies? 
Therapeutic approach and rationale: Mr. Smith has a specific lesion causing his back pain that is amenable to being 'fixed', so a reductionistic approach will likely be most helpful. An epidural markedly improved his symptoms, and he is now using occasional Naprosyn for residual pain and a course of Gabapentin if his sciatica flares. Opioids should generally be avoided since there is little evidence of benefit and much evidence of harm in treating most cases of CLBP. If his symptoms do not resolve over several months or worsen, neurosurgical intervention may be indicated.

Competencies for learners might include:

1. Appropriateness criteria for different radiologic studies in chronic low back pain

2. Indications and evidence base for different pharmaceuticals to treat somatic and neuropathic pain

3. Indications for interventions such as epidural injections and neurosurgical procedures

4. Indications and efficacy of different approaches to physical therapy

\subsection{Example 2}

Mr. Jones is a successful $45 \mathrm{y} / \mathrm{o}$ business man who c/o CLBP ranging from 3 to 8/10 for 7 months. He has no history of trauma or overuse, his back pain began insidiously at the time he was very involved in a stressful time in his business and had to work long hours in the office for several weeks. PMH is significant for obesity, gastroesophageal reflux disease (GERD), stage 1 essential hypertension (HTN) for which he takes Lisinopril and recently diagnosed prediabetes for which he takes Metformin. His only exercise is walking on the weekends. Sleep is occasionally disturbed. He states he has a standard American 'meat \& potatoes' diet and eats out often at fast food restaurants or snacks when working late in the office. Previous X-rays and MRI show several levels of mild disc bulges and mild facet arthropathy. Active range of motion (AROM) of lumbosacral (LS) spine is full and causes only minimal discomfort, inconsistent with his 5-8/10 recurrent pain experience. Para-lumbar spasm/ tightness and anterior pelvic tilt is noted. He has no red flags or neurological deficits on his evaluation.

1. Would you focus on a more reductionistic 'fix it' approach or a more holistic approach in this case? Why?

2. What are some ways Mr. Jones' eating habits may be related to his CLBP?

3. How might his exercise/conditioning status affect his CLBP?

4. How would you address his paralumbar spasm/tightness and anterior pelvic tilt?

5. How would you approach his request for a pill to help his pain? What might you choose first line?

6. Name 3 competencies that would be most relevant to learners in this case?

7. What would be general strategies in teaching these 3 competencies? 
Therapeutic program \& rationale: Mr. Jones has the common pattern of impending 'diabesity' (central obesity with metabolic syndrome spectrum of derangements), which is associated in studies with about a double risk of chronic pain. Stress likely worsens his pain. Deconditioning with core spine stabilizer muscle deficits and resultant reactive chronic para-lumbar and other related muscle tension may be a main driver of his pain. Poor correlation between spine imaging lesions and clinical symptoms makes a reductionistic 'fix it' approach to back care less likely to be successful.

A more holistic and lifestyle medicine approach with graduated exercise focusing on core strength and control and posture supported by PT, plant-based whole foods diet to reduce body inflammation, Somatics or similar modality to 'reconnect' mind/body control of paralumbar spasm to help relax these muscles and a comprehensive stress management/emotional support program are more likely to be beneficial. Natural botanicals that reduce inflammation such as Curcumin and/ or Boswellia may be useful to reduce inflammation and help prevent progression of prediabetes to DM2 and would not have clinically significant interactions with his present pharmaceutical regimen. NSAIDs would likely have an unacceptable risk/ benefit ratio by worsening GERD and HTN. Opioids generally have an unfavorable risk/benefit ratio for non-specific CLBP and should be avoided.

Competencies for learners in this case might include:

1. Understanding correlations between nutrition, obesity, DM2 and chronic pain and ability to communicate these risks and how to reverse them effectively to patients.

2. Familiarity with basic principles of both PT and mind/body modalities such as Somatics in treating CLBP and how to refer to specialists in these areas

3. Ability to guide patients on simple techniques to reduce stress, such as breathwork, progressive relaxation, meditation. Experiencing some of these techniques themselves may be a more useful learning approach than limiting to didactic knowledge.

\section{Motivational interview skills to support lifestyle change}

5. Basic knowledge and use of evidence-based botanical medicines for chronic pain

\subsection{Example 3}

Mrs. Patel is a 45 y/o second generation American with Asian Indian ancestry with CLBP ranging from 3 to $8 / 10$ for 7 months. The back pain started after she made a left turn and an oncoming car hit her at about $20 \mathrm{mph}$. She has no sciatica or red flag symptoms or findings. An X-ray done in the ER after the accident showed mild degenerative disc disease and spondylosis. Labs done in the ER were unremarkable except for mild elevated AST and an elevated MCV on CBC. Other health issues include migraines and irritable bowel syndrome and insomnia. She denies smoking or illicit drug use and states she drinks a 'nightcap' regularly to help with her sleep and pain. She struggles with marital difficulties with her Indian husband. She is sedentary, appears deconditioned and mildly centrally obese. She prefers not to exercise or move much because she fears it will worsen her back pain. Evaluation of the LS spine shows mild ache on flexion and far extension and some para-lumbar spasm and tenderness. No red flag findings. 
Teaching Balanced Patient Care Using Principles of Reductionism and Holism: The Example... DOI: http://dx.doi.org/10.5772/intechopen.82618

Study questions:

1. Would you focus on a more reductionistic 'fix it' approach or a more holistic approach in this case? Why?

2. How do Mrs. Patel's co-morbidities inform your evaluation of the causes and solutions to her CLBP?

3. To what degree would you estimate Mrs. Patel's spine x-ray correlates with her clinical findings?

4. How would you approach Mrs. Patel's fear of movement?

5. How might concerns regarding the likely or possible cause of Mrs. Patel's car accident, elevated AST \& MCV and self-treatments for pain inform your therapeutic approach?

6. Name 3 key competencies for learners in this case, using a reductionistic approach

7. Name 3 key competencies for learners in this case, from a holistic perspective

8. What would be key strategies in teaching each of these competencies?

Therapeutic program \& rationale: Ms. Patel would likely benefit from re-conditioning with PT to help her confidently establish healthy movement patterns. She needs education that chronic pain does not equal tissue damage in her case, and it is safe to move. NSAIDs, while possibly useful at least for a limited time, may have unacceptable risks with her possible alcohol abuse. Stress and related back muscle tension is a likely contributor to her pain and might be addressed by a combination of marital/psychotherapy, Somatics or similar approaches. She needs evaluation for possible alcohol dependency, anxiety and mood disorders. Magnesium and other nutritional deficiencies may aggravate her chronic pain, and she may benefit from supplementation.

Competencies for learners in this case might include:

1. Understanding early symptoms and signs of alcohol abuse (which is a major factor in motor vehicle accidents) and evaluation for same. Learning how to communicate concerns about substance abuse and facilitate steps toward change and how to refer.

2. Ability to analyze risk/benefit of NSAIDs and other pain medications.

3. Diagnosis and treatment of mood disorders and anxiety and effective referral for different types of counseling.

4. Teaching patients difference between acute and chronic pain, and how chronic pain does not equate with tissue damage. Effectively encouraging movement and exercise in chronic pain patients.

5. Cultural competency in relating to patients from different backgrounds. 


\section{Conclusion}

Chronic low back pain serves in this chapter as an example of the wide range of competencies along the reductionistic-holistic spectrum that may be needed in treating patients. Flexibility of therapeutic approach to match needs of the patient may improve outcomes. Although there is some evidence of better outcomes and value with such an integrative approach [55], more research is needed to test this hypothesis. Similarly, an integrative approach may help physicians reconnect with their reasons for going into medicine and their role as a healer. 'Walking the walk' by modeling a healthy lifestyle can restore their own health and reduce burn-out. There is little research on effective GME methods of teaching learners to balance reductionistic and holistic approaches to care, so present approaches are largely guided by experience. Given the expense and burden of suffering of chronic disease, this should be a priority area of research in GME.

\section{Acknowledgements}

We would like to recognize the Staff and Faculty at the St. Luke's Coventry Family Medicine Residency Program as well as the St. Luke's Post-doctorate research fellowship program for their ongoing support.

\section{Conflict of interest}

No conflicts of interests.

\section{Author details}

Alan Remde*, Stephen DeTurk and Thomas Wojda

SLUHN FMR-Warren, Phillipsburg, NJ, USA

*Address all correspondence to: alan.remde@sluhn.org

IntechOpen

(C) 2018 The Author(s). Licensee IntechOpen. This chapter is distributed under the terms of the Creative Commons Attribution License (http://creativecommons.org/licenses/ by/3.0), which permits unrestricted use, distribution, and reproduction in any medium, provided the original work is properly cited. (cc) BY 
Teaching Balanced Patient Care Using Principles of Reductionism and Holism: The Example... DOI: http://dx.doi.org/10.5772/intechopen.82618

\section{References}

[1] Reinhardt UE, Hussey PS, Anderson GF. U.S. health care spending in an international context. Health Affairs (Project Hope). 2004;23(3):10-25

[2] Martin BI, Deyo RA, Mirza SK, Turner JA, Comstock BA, Hollingworth W, et al. Expenditures and health status among adults with back and neck problems. Journal of the American Medical Association. 2008;299(6):656-664

[3] Services UDoHaH. Healthy People 2020 Leading Health Indicators: Progress Update. 2014

[4] Collaborators USBoD. The state of US health, 1990-2010: Burden of diseases, injuries, and risk factors. Journal of the American Medical Association. 2013;310(6):591-606

[5] Gearhardt AN, Corbin WR, Brownell KD. Food addiction: An examination of the diagnostic criteria for dependence. Journal of Addiction Medicine. 2009;3(1):1-7

[6] Komaroff AL. The microbiome and risk for obesity and diabetes. Journal of the American Medical Association. 2017;317(4):355-356

[7] Kreier F, Yilmaz A, Kalsbeek A, Romijn JA, Sauerwein HP, Fliers E, et al. Hypothesis: Shifting the equilibrium from activity to food leads to autonomic unbalance and the metabolic syndrome. Diabetes. 2003;52(11):2652-2656

[8] Komaroff AL. The microbiome and risk for atherosclerosis. Journal of the American Medical Association. 2018;319(23):2381-2

[9] Ludwig DS, Ebbeling CB. The carbohydrate-insulin model of obesity: Beyond "calories in, calories out". JAMA Internal Medicine. 2018;178(8):1098-1103
[10] Uribarri J, Woodruff S, Goodman S, Cai W, Chen X, Pyzik R, et al. Advanced glycation end products in foods and a practical guide to their reduction in the diet. Journal of the American Dietetic Association. 2010;110(6):911-916 e12

[11] Campbell TC, Junshi C. Diet and chronic degenerative diseases: Perspectives from China. The American Journal of Clinical Nutrition. 1994;59(5):1153S-1161S

[12] Krasner MS, Epstein RM, Beckman H, Suchman AL, Chapman B, Mooney CJ, et al. Association of an educational program in mindful communication with burnout, empathy, and attitudes among primary care physicians. Journal of the American Medical Association. 2009;302(12):1284-1293

[13] Ebell MH, Sokol R, Lee A, Simons $\mathrm{C}$, Early J. How good is the evidence to support primary care practice? BMJ Evidence-Based Medicine. 2017;22(3):88-92

[14] Kumar S, Nash DB. Health care myth busters: Is there a high degree of scientific certainty in modern medicine? Two Doctors Take on the Health Care System in a New Book that Aims to Arm People with Information. Scientific American. 2011

[15] MacPherson H, Hammerschlag R. Acupuncture and the emerging evidence base: Contrived controversy and rational debate. Journal of Acupuncture and Meridian Studies. 2012;5(4):141-147

[16] Ning Z, Lao L. Acupuncture for pain management in evidence-based medicine. Journal of Acupuncture and Meridian Studies. 2015;8(5):270-273

[17] Ross AC, Caballero B, Cousins RJ, Tucker KL, Ziegler TR. Modern Nutrition in Health and Disease. Lippincott Williams \& Wilkins; 2014 
[18] Franklin GM. Opioids for chronic noncancer pain. A position paper of the American Academy of Neurology. 2014;83(14):1277-1284

[19] Krebs EE, Gravely A, Nugent S, Jensen AC, DeRonne B, Goldsmith ES, et al. Effect of opioid vs nonopioid medications on pain-related function in patients with chronic back pain or hip or knee osteoarthritis pain: The SPACE randomized clinical trial. Journal of the American Medical Association. 2018;319(9):872-882

[20] Giles TD, Berk BC, Black HR, Cohn JN, Kostis JB, Izzo JL Jr, et al. Expanding the definition and classification of hypertension. Journal of Clinical Hypertension (Greenwich, Conn). 2005;7(9):505-512

[21] Boghossian TA, Rashid FJ, Thompson W, Welch V, Moayyedi P, Rojas-Fernandez C, et al. Deprescribing versus continuation of chronic proton pump inhibitor use in adults. The Cochrane Database of Systematic Reviews. 2017;3:Cd011969

[22] Pappas M, Jolly S, Vijan S. Defining appropriate use of proton-pump inhibitors among medical inpatients. Journal of General Internal Medicine. 2016;31(4):364-371

[23] Reimer C, Sondergaard B, Hilsted L, Bytzer P. Proton-pump inhibitor therapy induces acid-related symptoms in healthy volunteers after withdrawal of therapy. Gastroenterology.

2009;137(1):80-87 7.e1

[24] Vaishnav B, Bamanikar A, Maske P, Reddy A, Dasgupta S. Gastroesophageal reflux disease and its association with body mass index: Clinical and endoscopic study. Journal of Clinical and Diagnostic Research. 2017;11(4):OC01-OOC4

[25] Khanna R, MacDonald JK, Levesque BG. Peppermint oil for the treatment of irritable bowel syndrome: A systematic review and meta-analysis. Journal of Clinical Gastroenterology. 2014;48(6):505-512

[26] Lindfors $P$, Unge $P$, Arvidsson $P$, Nyhlin H, Björnsson E, Abrahamsson $\mathrm{H}$, et al. Effects of gut-directed hypnotherapy on IBS in different clinical settings-Results from two randomized, controlled trials. The American Journal of Gastroenterology. 2012;107(2):276

[27] Whitton PA, Lau A, Salisbury A, Whitehouse J, Evans CS. Kava lactones and the kava-kava controversy. Phytochemistry. 2003;64(3):673-679

[28] Breivik H, Eisenberg E, O’Brien $\mathrm{T}$. The individual and societal burden of chronic pain in Europe: The case for strategic prioritisation and action to improve knowledge and availability of appropriate care. BMC Public Health. 2013;13(1):1229

[29] Simon LS. Relieving pain in America: A blueprint for transforming prevention, care, education, and research. Journal of Pain \& Palliative Care Pharmacotherapy. 2012;26(2):197-198

[30] Janardhana AP, Rajagopal SR, Kamath A. Correlation between clinical features and magnetic resonance imaging findings in lumbar disc prolapse. Indian Journal of Orthopaedics. 2010;44(3):263

[31] Tonosu J, Oka H, Matsudaira K, Higashikawa A, Okazaki H, Tanaka $\mathrm{S}$. The relationship between findings on magnetic resonance imaging and previous history of low back pain. Journal of Pain Research. 2017;10:47

[32] Merskey HE. Classification of chronic pain: Descriptions of chronic pain syndromes and definitions of pain terms. Pain. 1986 
[33] Attal N, Lanteri-Minet M, Laurent B, Fermanian J, Bouhassira D. The specific disease burden of neuropathic pain: Results of a French nationwide survey. Pain. 2011;152(12):2836-2843

[34] Bair MJ, Robinson RL, Katon W, Kroenke K. Depression and pain comorbidity: A literature review. Archives of Internal Medicine. 2003;163(20):2433-2445

[35] Kroenke K, Outcalt S, Krebs E, Bair MJ, Wu J, Chumbler N, et al. Association between anxiety, healthrelated quality of life and functional impairment in primary care patients with chronic pain. General Hospital Psychiatry. 2013;35(4):359-365

[36] McWilliams LA, Goodwin RD, Cox BJ. Depression and anxiety associated with three pain conditions: Results from a nationally representative sample. Pain. 2004;111(1-2):77-83

[37] Gironda RJ, Clark ME, Massengale JP, Walker RL. Pain among veterans of operations enduring freedom and Iraqi freedom. Pain Medicine. 2006;7(4):339-343

[38] Higgins DM, Kerns RD, Brandt CA, Haskell SG, Bathulapalli H, Gilliam W, et al. Persistent pain and comorbidity among operation enduring freedom/ operation Iraqi freedom/operation new dawn veterans. Pain Medicine. 2014;15(5):782-790

[39] Lew HL, Otis JD, Tun C, Kerns RD, Clark ME, Cifu DX. Prevalence of chronic pain, posttraumatic stress disorder, and persistent postconcussive symptoms in OIF/OEF veterans: Polytrauma clinical triad. Journal of Rehabilitation Research \& Development. 2009;46(6)

[40] Qaseem A, Wilt TJ, McLean RM, Forciea MA. Clinical Guidelines Committee of the American College of Physicians. Noninvasive treatments for acute, subacute, and chronic low back pain: A clinical practice guideline from the American College of Physicians. Annals of Internal Medicine. 2017;166(7):514-530

[41] Lee CH, Chung CK, Kim CH. The efficacy of conventional radiofrequency denervation in patients with chronic low back pain originating from the facet joints: A meta-analysis of randomized controlled trials. The Spine Journal. 2017;17(11):1770-1780

[42] Juch JNS, Maas ET, Ostelo R, Groeneweg JG, Kallewaard JW, Koes BW, et al. Effect of radiofrequency denervation on pain intensity among patients with chronic low back pain: The mint randomized clinical trials. Journal of the American Medical Association. 2017;318(1):68-81

[43] Gibson JNA, Waddell G. Surgical interventions for lumbar disc prolapse. Cochrane Database of Systematic Reviews. 2007;2(1):CD001350. DOI: 10.1002/14651858.CD001350.pub3

[44] Conn A, Buenaventura RM, Datta S, Abdi S, Diwan S. Systematic review of caudal epidural injections in the management of chronic low back pain. Pain Physician. 2009;12(1):109-135

[45] Afari N, Ahumada SM, Wright LJ, Mostoufi S, Golnari G, Reis V, et al. Psychological trauma and functional somatic syndromes: A systematic review and meta-analysis. Psychosomatic Medicine. 2014;76(1):2

[46] Lumley MA, Cohen JL, Borszcz GS, Cano A, Radcliffe AM, Porter LS, et al. Pain and emotion: A biopsychosocial review of recent research. Journal of Clinical Psychology. 2011;67(9):942-968

[47] Hsu MC, Schubiner H, Lumley MA, Stracks JS, Clauw DJ, Williams DA. Sustained pain reduction through affective self-awareness in fibromyalgia: 
A randomized controlled trial. Journal of General Internal Medicine. 2010;25(10):1064-1070

[48] Lumley MA, Sklar ER, Carty JN. Emotional disclosure interventions for chronic pain: From the laboratory to the clinic. Translational Behavioral Medicine. 2011;2(1):73-81

[49] Slavin-Spenny O, Lumley MA, Thakur ER, Nevedal DC, Hijazi AM. Effects of anger awareness and expression training versus relaxation training on headaches: A randomized trial. Annals of Behavioral Medicine. 2013;46(2):181-192

[50] Lumley MA, Schubiner H, Carty JN, Ziadni MS. Beyond traumatic events and chronic low back pain: Assessment and treatment implications of avoided emotional experiences. Pain. 2015;156(4):565

[51] Garland EL, Manusov EG, Froeliger B, Kelly A, Williams JM, Howard MO. Mindfulness-oriented recovery enhancement for chronic pain and prescription opioid misuse: Results from an early-stage randomized controlled trial. Journal of Consulting and Clinical Psychology. 2014;82(3):448-459

[52] Kamper SJ, Apeldoorn AT, Chiarotto A, Smeets RJ, Ostelo RWJG, Guzman J, et al. Multidisciplinary biopsychosocial rehabilitation for chronic low back pain. Cochrane Database of Systematic Reviews. 2014;9:CD000963. DOI: 10.1002/14651858.CD000963.pub3

[53] Han DJ-S. Physiology of acupuncture: Review of thirty years of research. The Journal of Alternative and Complementary Medicine. 1997;3(Suppl. 1): s-101-s-8

[54] Furlan AD, van Tulder MW, Cherkin D, Tsukayama H, Lao L, Koes BW, et al. Acupuncture and dry-needling for low back pain.
Cochrane Database of Systematic Reviews. 2005;(1):CD001351. DOI: 10.1002/14651858.CD001351.pub2

[55] Sundberg T, Petzold M, Kohls N, Falkenberg T. Opposite drug prescription and cost trajectories following integrative and conventional care for pain-A case-control study. PLoS One. 2014;9(5):e96717 


\title{
Chapter 6
}

\section{Physician Burnout}

\author{
Bess Connors, Charlotte Horne, Valery Vilchez \\ and Sofya Asfaw
}

\begin{abstract}
Burnout is pervasive among physicians and is rapidly becoming a pandemic in healthcare. It is characterized by increasing demands without adequate support and hallmarked by depersonalization, emotional exhaustion, and a reduced sense of personal accomplishment. It is essential to address burnout, as it can lead to decreased productivity, increased healthcare costs, medical errors, workforce attrition, depression, and even suicide. Many factors contribute to burnout, and it occurs at all stages of medicine: it can begin during medical school, intensify during the years of graduate medical education (GME) or residency training, and persist as residents become staff physicians. It affects both sexes, but may impact female physicians disproportionately. Impact can also vary among specialties. Recognizing the problem and intervening with unified physician and organization-directed solutions centered on well-being, efficient practice models, and goal prioritization may help to reduce the prevalence and effects of burnout.
\end{abstract}

Keywords: burnout, depersonalization, emotional exhaustion, suicidal ideation, attrition, patient safety, resilience, well-being

\section{Introduction}

Physician burnout is a quiet pandemic in health care. Its negative effects infiltrate the lives of those experiencing it and also negatively impact patient outcomes, increase medical errors, and decrease overall productivity. The number of physicians that experience burnout is staggering, with rates quoted from 50 to $67 \%[1,2]$. This is significantly higher than those seen in the general population, including occupations holding a professional degree [3]. Burnout is not only an issue of practicing physicians, but it is also common during graduate medical education and is becoming prevalent in medical students-even in their first year of study $[4,5]$. An increasing amount of attention is being given to this issue due to the high prevalence in physicians in the USA and globally. Although rates of burnout differ between disciplines and gender $[6,7]$, it is pervasive in all types of medical specialties. Due to the prevalence of burnout in all stages of training, implementing programs focusing on awareness have become a focus in graduate medical education. Unfortunately, despite an increased awareness, burnout rates continue to rise [8]. Numerous factors have been associated with this including increased utilization of the electronic medical record, changes in physician payment structure, increasing workload despite resource deficits, increasing administrative burdens, and overall lack of autonomy. 
1. I feel emotionally drained from my work.

2. I have accomplished many worthwhile things in this job.

3. I don't really care what happens to some patients.

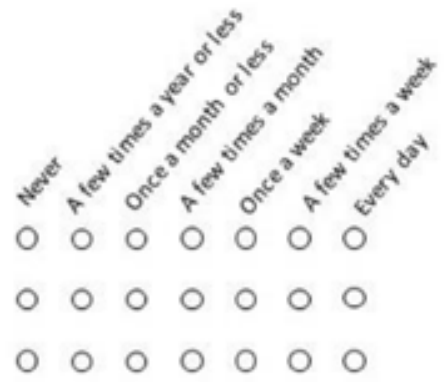

Figure 1.

Sample MBI questions. MBI-HSS: Copyright $@ 1981$ Christina Maslach and Susan E. Jackson. All rights reserved in all media. Published by Mind Garden, Inc., www.mindgarden.com.

Burnout was described by Freudenberger in 1974 as chronic stress due to intense work demands in the setting of inadequate resources [9]. It was further characterized by Maslach et al. based on three components: a state of depersonalization, emotional exhaustion, and reduced personal accomplishment [10]. When emotional exhaustion contributes to burnout in providers, they feel as though they no longer have the emotional capability to care for patients. Depersonalization results in cynical feelings toward patients, colleagues, or both. Personal accomplishment is decreased when physicians experience less satisfaction from or perceive less value in completed tasks. High levels of emotional exhaustion and depersonalization combined with low levels of personal accomplishment result in burnout. More recently, DeCaporale et al. described it as "an emotional manifestation of a profound mismatch between high expectations for one's future and the reality of daily life" [11].

Not only does burnout negatively affect overall work productivity and patient care, but burnout has been closely associated with depression [12] and other mental health issues. The rate of suicide and suicidal ideation is higher among physicians than in the general population [13], and physicians are more likely to commit suicide than non-physicians [14]. Addressing burnout is essential, as 400 physician lives are lost to suicide each year in the USA alone [14]. It is important to understand the factors that lead to burnout in students, GME trainees, and staff physicians and to implement strategies to address them.

\subsection{Measuring burnout}

The most common method of assessing burnout is based on the Maslach Burnout Inventory (MBI) (Figure 1). First introduced in 1981, the MBI is an externally validated survey that measures dimensions of burnout including emotional exhaustion and depersonalization. Using language that elicits personal attitudes, respondents evaluate statements indicating how frequently they relate to the statement using a 7-point scale. Higher-scored responses in the emotional exhaustion and depersonalization sections correspond to a higher likelihood of burnout, while lower scores in the personal accomplishment subscale indicate increased risk of burnout.

\section{Factors contributing to physician burnout}

Work in the medical field provides unique challenges. From early in GME training and even medical school, student doctors are exposed to death and human suffering on a daily basis. Ethical conflicts also present themselves-some with no 
satisfactory solution. The work has long hours, and there are ever-increasing clerical requirements. As such, factors thought to contribute to physician burnout are numerous: some include work-related factors at the individual and organizational levels, as well as more personal factors which may leave some physicians predisposed to burnout.

The era of the electronic health record (EHR) has arrived, and along with the myriad benefits, physicians are feeling the negative effects. Many physicians now spend a greater proportion of their time documenting care than providing it face-to-face with patients [15]. In the USA, documentation has become onerous due to requirements of legal compliance and billing. In the development of the EHR, financial and compliance features came first and often at the cost of usability. The division of physician time and attention between patients and the EHR has led to less fulfilling relationships with patients and a growing concern that the EHR may drive dissatisfaction and burnout. Electronic order entry alone was associated with a $29 \%$ increase in physician burnout [16]. The EHR is not the only example of workplace inefficiencies adding to burnout. Inefficient processes and clerical work not contributing to meaning in a physician's work likely contribute to burnout as well.

This loss of "meaning in work" is difficult to define yet is a strong contributor to dissatisfaction. Physicians who spend less than $20 \%$ of their work effort on the activity they find most personally meaningful are nearly three times more likely to be burned out than those who spend at least $20 \%$ of their work effort on such an activity [17]. It is possible that the shift in physician employment structures from private practice to employed providers has contributed to a loss of autonomy and diminished ability to choose one's work activities. This may contribute to the reported loss of meaning in work. When leadership makes a greater effort to seek input from physicians, recognize their individual contributions, and grant them greater control in workplace matters, physicians show greater levels of satisfaction and lower levels of stress [18]. The protective effect suggests that loss of autonomy is associated with increased rates of burnout.

The more a physician works, the more likely she will suffer from burnout. Multivariable analyses of data from cross-sectional studies of physicians have reported independent relationships between burnout and work hours, call shifts, time spent on work from home, and the presence of work-home conflicts. As an example, the presence of work-home conflicts more than doubles the incidence of burnout [2]. Unfortunately, many hospital systems are responding to small financial margins and patient access issues by demanding that physicians see and care for ever-increasing numbers of patients, necessitating longer work hours. The cycle will not be sustainable as burnout will lead more physicians to leave the workforce or reduce hours-seeing fewer patients.

Finally, it seems as though nearly any demographic collected has led to observed differences in rates of burnout. Independent relationships between burnout and physician sex, age, educational debt, relationship status, age of children, and spousal/partner occupation have all been reported [2]. Special attention has been paid to burnout during the different stages of physician training, where it is seen in both undergraduate and graduate medical education. Individual personality, personal experiences, and interpersonal skills may impact coping mechanisms and resilience among physicians; however, it does not appear that those entering the medical field are more predisposed or vulnerable to stress and burnout than the general population [19]. It is something about the work, and not the worker, that is defective. Some special cases including burnout across genders, stages of training, and in the surgical specialties will be discussed further. 


\section{Special cases}

\subsection{Gender differences in burnout}

Female physicians have been shown to experience burnout at a higher rate than males [7]. As females currently make up close to $50 \%$ of medical graduates, compared to just $8 \%$ in 1965 [20], gender disparity in burnout is likely to have an increasing effect as more women enter training and become practicing physicians. Some of the proposed factors influencing higher burnout rates are differences in work-life balance and gender roles women are expected to fulfill both in and out of the hospital.

A study comparing female to male surgeons by Dyrbye et al. explored certain demographic and social factors in an effort to explain why female doctors were more likely to become burned out. Factors that were likely to affect work-life balance including age, number of children, marital status, and other partner characteristics were compared between male and female surgeons. The study found that female physicians were more likely to be married to a spouse that also worked outside home compared to the male colleagues [7]. The spouses of these women were not only more likely to be doctors but also more likely to be surgeons when compared to their male colleagues. Despite more commonly being involved in situations where both partners were working, often in equally demanding careers, women played a more significant role in child rearing than their male colleagues.

Dyrbye went on to further evaluate how personal achievement may be different between genders. They demonstrated that compared to male surgeons, female surgeons felt that child rearing slowed their career advancement. In partnerships where both individuals were employed and especially in the case where both were employed as physicians, conflicts arose between the careers of the two individuals. These career conflicts were more common for women and less likely to resolve in their favor. Career conflicts were more likely to resolve in favor of the surgeon when the surgeon was male compared to when the surgeon in the partnership was female [7].

Female physicians' roles in their home life differ significantly from those of their male colleagues, but their roles in the hospital also differ. Female physicians have been found to treat a distinct patient population, and the expectations women face from those patients is also somewhat different from those faced by their male colleagues. This may begin to explain the higher incidence of burnout in women. Female physicians see a higher number of patients with more complex psychosocial issues, and patients often expect female physicians to be more empathetic [21, 22]. This combination of more complex issues arising and increased expectations of empathy from female providers leads to longer office visits [23]. Female physicians' appointment duration has been shown to be $10 \%$ longer that than that of their male colleagues despite being assigned equal clinic time slots [24]. Female physicians face difficult time constraints: as appointments risk running over, these women find they are also more likely than their male counterparts to be evaluated negatively in the event that they end the encounter sooner than the patient would like. The increasing pressure to remain empathetic, address all patient concerns, conduct clinic efficiently, provide appropriate patient care, support a significant other, and play a major role in child rearing, all while attempting to advance her career likely causes a significant amount of emotional exhaustion which may lead to higher rates of burnout for female physicians.

\subsection{Burnout in training}

\subsubsection{Burnout in medical school}

Burnout is not limited to those that have been in practice for numerous years. Even medical students in their first year of training report experiencing burnout. 
Santen et al. showed that as many as one third of medical students displayed a moderate to high degree of burnout, with rates of burnout as high as 41 and $43 \%$ in the second and third years of medical school, respectively [25]. There are numerous transitions that medical students experience throughout their education which may result in burnout. In the first 2 years of school, significant classroom responsibilities and pressure to perform well on tests as well as to retain pertinent information can be overwhelming. As the student transitions to their clinical years, feelings of decreased personal accomplishment; the transition to a clinically based education; rotation interactions with various physicians, residents, and patients; as well as the residency application process may increase emotional exhaustion and depersonalization.

In the first 2 years of medical school, burnout is strongly associated with the amount of support perceived by the student from their faculty [26]. As students transition to clinical practice and prepare residency applications in their third year of training, emotional exhaustion and depersonalization peak. It is this year that students report the highest degree of burnout $[25,26]$. Reasons for this include exposure to cynical residents, the organization of clerkship rotations, and increased hours spent in the hospital due to the requirement to take overnight call [27].

Life events experienced during medical school may also contribute to the presence or absence of burnout in students. Using survey data from medical students within Minnesota, Dyrbye et al. evaluated how either positive or negative life events affected overall rates of burnout in medical students. Like their peers, students in medical school have to deal with personal or family illness or death as well as marriage or the birth of a child. However, unlike their peers, these must be handled when schedules are less than flexible. Often, medical students are required to relocate away from their families to pursue their education, which can make coping with personal or family illness exceedingly difficult. Mental health has been shown to deteriorate during medical school. Burnout in medical students is extremely worrying due to the high association between burnout and suicidal ideation. Approximately $11 \%$ of medical students report suicidal ideation [27]. Students that reported feeling burnout were up to three times more likely to consider suicide. Students with high rates of depersonalization were especially at risk for suicidal ideation.

\subsubsection{Burnout in graduate medical education}

Residency is a challenging time regardless of which specialty a resident chooses. During this time, residents must obtain a significant amount of clinical knowledge, balance daily patient care activities, and deal with difficult complications, all with little control over their schedules. As many as $69 \%$ of surgical residents meet the criteria for burnout and $50 \%$ of surgical residents report high emotional exhaustion [28]. Medicine residents were similarly affected with as many as $76 \%$ of residents feeling burned out [29].

Long work hours, multiple call shifts, and lack of sleep are key features of residency that have been evaluated as potential causes of burnout. Other possible factors that may result in high degrees of burnout in residency are low sense of personal accomplishment due to feelings of decreased self-efficacy and competency. Purdy et al. surveyed family medicine residents to catalog perceived causes for burnout in residency. The residents in this survey reported significant time constraints as the major factor leading to burnout [30]. These findings were confirmed in a study involving anesthesia residents that cited lack of time but additionally noted lack of control over time management, inherently difficult job situations, and interpersonal conflicts as contributing causes to burnout [31]. Although residents reported that feeling overwhelmed was likely to increase the risk of burnout, it did 
not correlate with total hours at work but rather with the number of emergency admissions and patient deaths [32].

Self-efficacy may also play a significant role in the overall resident well-being. Achieving self-efficacy often correlates to the confidence that one has mastered a certain skill, and this appears especially true in specialties that require a significant amount of technical instruction [33]. As adjustments to duty hour restrictions have decreased total hours residents spend in the hospital, residents' perception of their self-efficacy and overall well-being may also decrease. Milam et al. evaluated the relationship between self-efficacy and well-being in a cohort of surgical residents. They found that residents that had high levels of self-efficacy reported less emotional exhaustion as well as higher levels of personal accomplishment, which in turn decreased burnout [34].

\subsection{Burnout in surgery}

Surgeons not only have a technically demanding career, but changes in delivery of medicine have transitioned to a larger amount of administrative work. Rates of burnout in surgeons are rising, and greater than $50 \%$ of surgeons report feeling burned out in some surgical subspecialties [8]. Factors specific to the practice of culture of surgery may put surgeons at risk for burnout.

Complications are common in surgery. Bosk stated that "the specific nature of surgical treatment links the action of the physician and the response of the patient more intimately than in other areas of medicine" [35]. Despite complications being a reality of the practice of surgery, often the emotional aspects of dealing with difficult complications are not routinely addressed. Luu et al. contend that there is a significant impact on the healthcare provider after an adverse event. Adverse events can lead to burnout and depression [36]. Often the necessary steps are not taken to reconcile with the complication. Increasing time constraints due to clinical demand do not allow the time to resolve these emotional disturbances after a complication, and surgeons may feel they need to "get back on the horse" as quickly as possible [36].

The culture of surgery also reinforces that significant emotional events should be handled privately. A study conducted by Lemaire and Wallace reported that the three most common coping mechanisms employed by surgeons include keeping stress to oneself, concentrating on what to do next, and going on as if nothing happened [37]. Having to silently confront significant complications on top of a demanding clinical schedule and incentive-based pay structures may contribute to the high degree of burnout seen in the surgical community.

\section{Impact of physician burnout}

Burnout has numerous effects on those who suffer from it. Outside the workplace, providers with burnout may suffer from marital problems, depression, substance abuse, and even suicide [11]. Burnout is also associated with negative health implications including sleep disturbances, hypertension, anxiety, and myocardial infarction [38]. Burnout not only affects the personal lives of those who suffer from it, but it also results in increased callousness and lack of empathy toward patients and can result in less attentiveness to patient care. This can lead to increased adverse events and errors [39-41]. The result is decreased patient satisfaction scores, increased medical error, increased short-term and long-term disabilities of those suffering from burnout, and an increase in physician turnover as providers leave patient care positions in efforts to mediate symptoms of burnout. 


\subsection{Consequences to physician health}

When examining the effects of burnout on physician health, it can be difficult to tease apart cause from correlation. The very question of whether burnout is a unique entity, or rather a form of depression, is debated. In a study that directly compared depressive symptoms in burned out workers and clinically depressed outpatients using a DSM-based approach, no diagnostically significant difference was observed between the two groups [42]. The team concluded that no burned out participant appeared to be free of depressive symptoms. At a symptom level, it has been difficult to isolate substantial differences between depression and burnout [12]. What probably matters more than the exact distinction between the two entities is their shocking prevalence and strong correlation that has been shown in numerous studies $[43,44]$.

Among the three most studied components of burnout, emotional exhaustion shows the strongest link to depression with moderate to high correlations. The link of depression with depersonalization and reduced personal accomplishment tends to be weaker [42].

Burnout is also strongly correlated with substance abuse in physicians [45]. In a study which surveyed surgical fellows only, alcohol abuse was found at a rate of $13.9 \%$ for male surgeons and $25.6 \%$ for female surgeons. Since women in the general population typically see reported rates of alcohol abuse half those of their male counterparts, these numbers are particularly concerning for the female surgeon group.

Physicians are also at high risk for suicidal ideation and death by suicide beginning as early as medical school [4]. The suicide rate among male physicians is more than $40 \%$ higher than that found among men in the general population, and the suicide rate for female physicians has been predicted as much as $130 \%$ higher than that of the general female population [46]. While the male suicide rate is four times the female rate in the general population, in the physician group, men and women have equivalent rates of suicide. The association between elevated suicide risk in physicians and prevalence of burnout has been investigated, with the presence of burnout appearing to double the risk of suicidal ideation [47].

\subsection{Consequences to patient safety}

After the 1999 Institute of Medicine report suggested that medical errors were a leading cause of preventable death in the USA, patient safety came to the forefront of healthcare policy [48]. Despite hard work in this area, medical errors by physicians remain a common cause of morbidity and mortality. Burnout has been linked with medical errors, and its presence doubles reported rates of medical errors [39]. Associations between burnout and medical errors do not prove causality, but medical errors are definitely a source of distress for physicians [49]. This is the basis for the "second victim" theory-providers who feel personally responsible for poor patient outcomes can become traumatized by these events. There is, however, increasing evidence that there is a bidirectional relationship between medical errors and physician distress. The longitudinal Internal Medicine Resident Well-Being (IMWELL) study found not only that self-perceived major medical errors were associated with worsening burnout, depressive symptoms, and decrease in quality of life but also that higher levels of burnout were associated with increased odds of reporting a major medical error in the subsequent 3 months [50].

The reported impact of medical errors and suboptimal care on patient morbidity and mortality is disturbingly high. In a review of 111 deaths at seven Veterans Affairs medical centers, $22.7 \%$ of active-care patient deaths were rated as at least 
“possibly preventable by optimal care," with 6.0\% rated as "probably or definitely preventable" [51]. This is similar to previously reported rates [52, 53]. If, as stated above, burnout doubles the risk of medical errors, and medical errors have a high impact on patient mortality, then burnout should be considered a major issue for public health. In the Minimizing Error, Maximizing Outcome (MEMO) study, a conceptual model was used to examine whether stressed, dissatisfied, and burned out physicians deliver poorer quality care. In a self-report, these physicians do demonstrate a greater likelihood of making errors and more frequent instances of suboptimal patient care [54]. This aligns with earlier studies, one of which found $75 \%$ of the resident population examined met criteria for burnout and that these burned out residents were two to three times more likely to report suboptimal patient care practices [55]. Beyond increased hospital morbidity and mortality, this suboptimal care in the hospital setting may have other long-term effects. Patients who are cared for by burned out physicians experience longer recovery times post-discharge. This effect was tied in particular to depersonalization, a hallmark of burnout [56]. There is also an increased likelihood that burned out surgeons will be named in a malpractice suit (17\% increase) [2]. One might imagine this could be due to the combination of increased medical errors and depersonalization leading to unsatisfactory communication and, thus, poorer relationships with patients. Patients do report lower satisfaction with their medical care and show poorer adherence to the doctor's recommendations when their physician is suffering from burnout [57]. Compromised physicians lack the capacity to build the personal trust and rapport in relationships with their patients that leads to improved compliance and patient satisfaction with their care.

\subsection{Consequences to the healthcare system}

Cross-sectional studies have associated physician burnout with decreased productivity, job dissatisfaction, and a more than doubled self-reported intent to leave one's current practice for reasons other than retirement [58]. Some physicians do leave the work force, and the cost of replacing them, from recruitment to lost productivity while the position is vacant, can total well over 1 million dollars in some markets. For those physicians that push through burnout, many will reduce work hours and workload. In a study published in 2016, a one point increase in emotional exhaustion or decrease in work satisfaction on a 7-point scale was associated with greater likelihood of reducing work hours (measured by administrative FTE data) [40]. Even those who do not officially leave the workforce or decrease hours demonstrate greater absenteeism and lower productivity [41]. At a time when physician shortages loom, decreased productivity in the physician workforce represents a serious threat to our ability to provide care to patients in need. Aside from the cost incurred through attrition and lost productivity, there are other ways in which burnout may impact the ballooning cost of medical care in the USA-medical errors and malpractice claims, both discussed above, are very costly. There is some evidence that physicians experiencing burnout or high workloads also have higher rates of referral to specialists and the use of diagnostic tests which may translate into additional costs for patients and insurers [59].

\section{Recognizing the problem}

Clinician well-being is crucial for safe, high-quality patient care. As we have described previously, burnout is associated with serious risks to both physicians and patient care; thus, it is imperative that healthcare providers have access to 
evidence-based interventions that reduce the risk for burnout. It is essential to understand that when physicians are burned out, healthcare system performance is suboptimal. Awareness of this problem in the healthcare community is essential. The difficulty with this subject is recognizing the drivers and applying timely interventions.

Interventions for burnout have been classified into two main categories: physician-directed and organization-directed, with the latter often targeting the working environment. Physician-directed interventions typically involve mindfulness or cognitive behavioral techniques to identify burnout symptoms early, improve communication skills, and augment personal coping strategies. Organizationdirected interventions can involve simple changes in schedule or workload or more ambitious changes in operation of practices within the institution. These usually involve improved teamwork, supervision to reduce job demand, enhanced job control, adjusted work-life balance, and increasing the level of participation in decision-making or leadership among the healthcare providers [60, 61].

Despite some limitations (including focus on single interventions rather than combined approaches, limited application of randomized and controlled designs, difficulties in generalization of results across specialties), intervention studies have demonstrated reductions in the proportion of physicians with burnout symptoms.

A meta-analysis by West et al. evaluated outcomes of potential intervention for burnout. Individual burnout domain scores were scaled to the relevant full Maslach Burnout Inventory range [60]. The authors report an overall burnout decrease of $10 \%$ (from 54 to $44 \%$ ), emotional exhaustion score decrease of 2.65 points in 36 studies, and depersonalization score decrease of 0.64 points in 36 studies.

Panaglioti et al. performed a meta-analysis to evaluate the effectiveness of interventions to reduce physician burnout and examine whether different types of interventions, physician characteristics, or healthcare settings were associated with decreased burnout symptoms. Three prespecified subgroup analyses were carried out: (1) physician-directed vs. organization-directed interventions, (2) physician working experience of greater than or less than 5 years, and (3) primary care vs. secondary care settings. The authors found that interventions were associated with small but significant reductions in burnout. Of note, the effects of organization-directed interventions were significantly larger than the effects of physician-directed interventions [62]. The results also suggested that interventions targeting experienced physicians and delivered in primary care setting showed greater effectiveness when compared with interventions targeting less experienced physicians and delivered in secondary care settings.

\subsection{Resident wellness in graduate medical education (GME)}

Burnout, depression, and suicidality among residents of all specialties have become a critical focus for the medical education community, especially among learners in GME. In 2017, the Accreditation Council for Graduate Medical Education (ACGME) updated the Common Program Requirements to focus more on resident well-being. These changes establish a mandate to educate residents and faculty members in the identification of burnout, depression, and substance abuse and to implement programs that encourage optimal resident and faculty well-being. Some of the programs established by the ACGME include (1) Clinical Learning Environment Review CLER Program which provides sponsoring institutions with periodic feedback from site visits addressing duty hours, fatigue management and mitigation, as well as well-being, (2) back to bedside initiatives to empower residents to develop projects that address burnout by fostering meaning in their learning environments, and (3) Action Collaborative on Clinician Well-Being 
and Resilience in conjunction with the American Association of Medical Colleges (AAMC) and the National Academy of Medicine (NAM).

\subsection{Trial interventions and effects}

Evidence has linked 1-point changes in burnout scores with meaningful differences in self-perceived major medical errors, reductions in work hours, and suicidal ideation. These concerns have prompted calls for increased attention to physician well-being, including efforts targeting burnout [60, 61].

Effective physician-directed strategies include mindfulness-based approaches, stress management training, small group curricula, belonging intervention, and Balint intervention. Balint intervention, a method which came to prominence in the 1950s in England, allows groups of practitioners to focus on the emotional content of doctor-patient relationships through case presentation followed by general discussion with emphasis placed on emotional reaction. Effective organizational approaches include duty hour regulations, shortened attending rotation length, and locally developed modifications to clinical work processes (Table 1 ).

Medical training, particularly residency, poses many challenges that may lead to burnout. The ACGME implemented mandated nationwide restrictions on resident work hours as of July 1,2003 . The new requirements include an 80 -hour weekly limit, averaged over 4 weeks, at least 8 hours of rest between duty periods, a

\begin{tabular}{|c|c|c|}
\hline Driver & Organization-level solutions & Individual-level solutions \\
\hline $\begin{array}{l}\text { Excessive } \\
\text { workload }\end{array}$ & $\begin{array}{l}\text { - Duty hour limits } \\
\text { - Appropriate distribution of job roles } \\
\text { and time needed for each role }\end{array}$ & $\begin{array}{l}\text { - Part-time status } \\
\text { - Informed practice choices }\end{array}$ \\
\hline $\begin{array}{l}\text { Work } \\
\text { inefficiency }\end{array}$ & $\begin{array}{l}\text { - Optimized EMS } \\
\text { - Appropriate interpretation of regulatory } \\
\text { requirements }\end{array}$ & $\begin{array}{l}\text { - Skill training sessions } \\
\text { - Delegate work appropriately }\end{array}$ \\
\hline $\begin{array}{l}\text { Lack of work- } \\
\text { home integration }\end{array}$ & $\begin{array}{l}\text { - Respect for home responsibilities in } \\
\text { setting office and meeting schedules } \\
\text { - All required work tasks should be able } \\
\text { to be completed within expected work } \\
\text { hours } \\
\text { - Support flexible work schedules }\end{array}$ & - Attention to self-care \\
\hline $\begin{array}{l}\text { Loss of } \\
\text { autonomy } \\
\text { Loss of meaning } \\
\text { from life }\end{array}$ & $\begin{array}{l}\text { - Physician leadership and shared } \\
\text { decision-making }\end{array}$ & $\begin{array}{l}\text { - Stress management and resiliency } \\
\text { training } \\
\text { - Positive coping strategies } \\
\text { - Mindfulness }\end{array}$ \\
\hline $\begin{array}{l}\text { Loss of meaning } \\
\text { from work }\end{array}$ & $\begin{array}{l}\text { - Promote shared core values } \\
\text { - promote physician communities } \\
\text { - Protect physician time with patients } \\
\text { - Professional development opportunities } \\
\text { - support marginalized groups } \\
\text { - Leadership training } \\
\text { - Awareness around physician burnout }\end{array}$ & $\begin{array}{l}\text { - Self-awareness of most fulfilling } \\
\text { work roles } \\
\text { - Mindfulness } \\
\text { - Promote sharing work experiences } \\
\text { - Belonging/Balint }\end{array}$ \\
\hline
\end{tabular}

Adapted from: West et al. [2].

Table 1.

Drivers and selected solutions for physician burnout. 


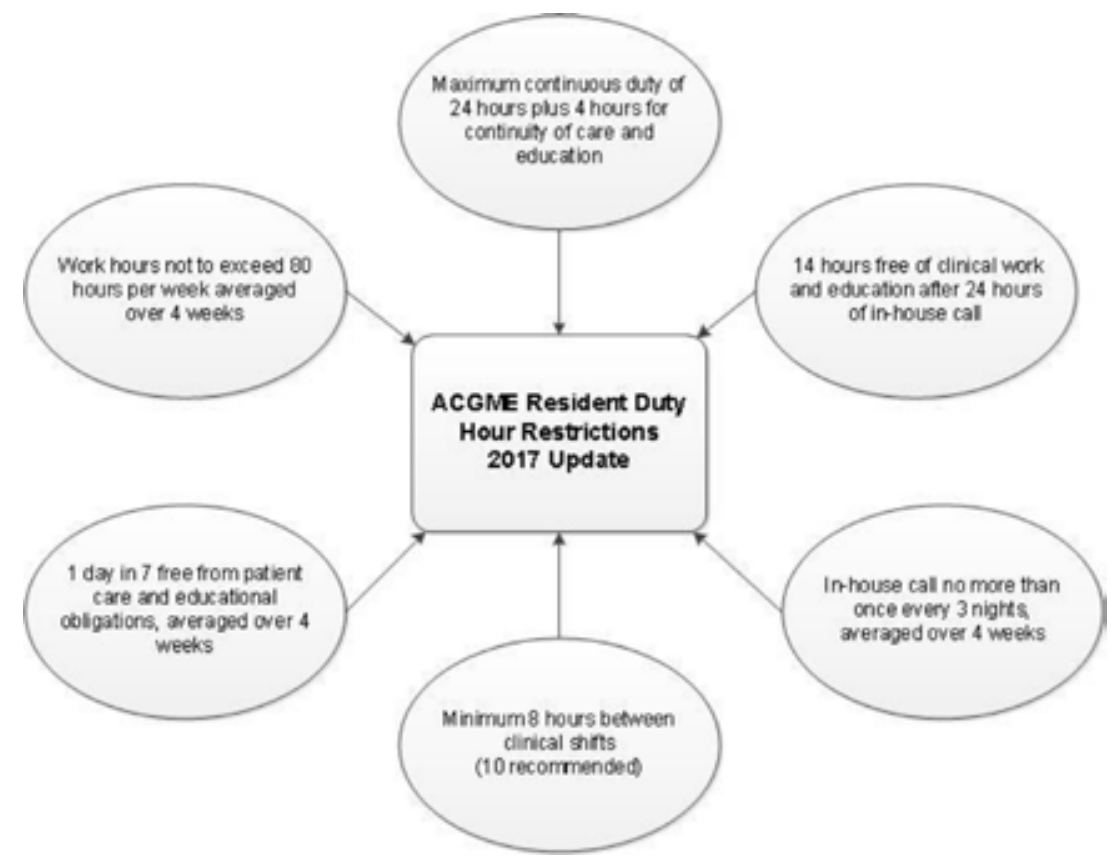

Figure 2.

ACGME clinical work hour common program requirements.

24-hour limit to continuous duty, 1 day in 7 free from patient care, and in-house call no more than once every 3 nights averaged over 4 weeks (see Figure 2 ).

Studying the impact of the 80 -hour resident workweek on surgical residents and attending surgeons implemented by the ACGME in a single institution, Hutter et al. reported that surgical residents had decreased burnout scores, with significantly less emotional exhaustion (Maslach Burnout Inventory (MBI): 29.1 "high" vs. 23.1 "medium," p 0.02). Residents seem to have better quality of life both in and out of the hospital: they reported getting more sleep, lighter workload, and increased motivation to work (Herzberg Motivation Dimensions). However, for attending surgeons, MBI scores on personal accomplishment, emotional exhaustion, and depersonalization resulted in the "medium" range for every category at both time points, showing no significant change after duty hour restrictions [63].

Salles et al. [64] studied the effect of the belonging intervention on surgical resident attrition. The authors included participants from seven surgical specialties who were randomized into either belonging treatment or control condition. Junior residents read anecdotes from senior residents describing the challenges the seniors had faced early in residency (treatment condition) or describing challenging ethical dilemmas they had encountered (control condition). Juniors were then asked about their attitudes and beliefs as a proxy for future intent to leave residency. The authors reported that residents who felt a stronger sense of belonging were more likely to think they would complete residency. After the intervention, residents in the treatment condition had significantly lower rates of burnout than those in the control condition.

Formal implementation of a program to improve resident well-being has been proven to positively impact residents' perceived stress, emotional exhaustion, emotional intelligence, life satisfaction, and their perception of the residency program [65]. A group from the University of Arizona created a program designed to improve surgical resident well-being named Energy, Leadership, Well-Being and Resiliency Program. Forty-nine general surgery residents participated in the program. They were assessed at baseline and 1 year after implementation using 
the Energy Leadership index, which measures emotional intelligence, MBI score, Perceived Stress Scale, the Beck Depression Inventory, and the annual required ACGME resident survey. The authors reported that 1 year after implementation, resident scores on the Energy Leadership Index improved, residents' perceived stress decreased from baseline, and scores on the emotional exhaustion scale of the MBI decreased. Resident-reported satisfaction increased in many areas over the 1 year interval: satisfaction with leadership skills, work relationships, communication skills, productivity, time management, and work-life balance.

\subsection{Listen-Act-Develop model: the Mayo Clinic experience}

The Listen-Act-Develop model is a physician engagement model that has been established by the Mayo Clinic and is practiced as a core value. This model is based on organization-sponsored practice improvement initiatives created, developed, and implemented by staff members $[66,67]$.

The four steps in the process are: (1) Listen: to actively seek specific burnout drivers and create a mitigation plan focused on the driver of greatest concern; (2) Act: to identify physician leaders and work with them to create solutions, facilitate implementation, monitor outcomes, and finally communicate results of the initiative to all staff members; (3) Develop: to support physician leadership development with mentoring, assessment, assignments, and goal planning; and (4) Repeat: to revisit findings from focus groups to identify the next round of initiatives to decrease specific burnout drivers

Since 2013, the institution's board of governors has sponsored an in-depth burnout engagement initiative with physicians from seven high-opportunity clinical departments. Initially, a team of two physicians and one administrator met with the division or department chair to obtain his or her opinions regarding local challenges and issues. They then conducted multiple focus groups with the members of the division or department and summarized the areas of greatest concern for the division or department chair. The institutional drivers of burnout identified through this process were communicated to the appropriate management groups and addressed by means of a department-organization partnership. A Burnout Mitigation Process Playbook was developed out of this process which has been used to facilitate the burnout mitigation process [68]. Follow-up surveys of physicians in these seven divisions and departments showed a median burnout reduction of 11 percentage points. After administration of the 2013 all-staff survey, work units were identified on the basis of lower scores for engagement and overall satisfaction ( $<75 \%$ on overall satisfaction and $<78 \%$ on engagement). Fifty percent of the targeted units experienced an increase in satisfaction, engagement, or both of these after actions were implemented [66].

\subsection{A unified approach}

In 2017, the NAM in conjunction with GME and AAMC introduced the Action Collaborative on Clinician Well-Being and Resilience, a network of more than 60 organizations committed to battle clinician burnout. There are three main goals of this action collaborative: (1) identify and actively seek signs of clinician anxiety, depression, stress, and suicide, (2) improve understanding of challenges to clinician well-being, and (3) advance multidisciplinary solutions to improve patient care by caring for the caregiver. Five working groups will meet over the course of 4 years to identify evidence-based strategies to improve clinician well-being at both the individual and systems levels. Activities of these groups include a series of NAM perspective discussion papers and a model that reflects the most important domains 
affecting clinician well-being [69]. This is an example of the unified approach necessary to address the problem of physician burnout.

\subsection{Moving forward}

Burnout is prevalent among physicians. Meaningful progress requires combined efforts by national organizations and individual physicians, as each is responsible for factors that contribute to the problem and must own their part of the solution.

Many knowledge gaps still remain in the understanding of physician burnout. It is necessary to continue following the outcomes of interventions aimed at mitigation and prevention of burnout that have been published in the literature. The effect of physician-oriented and organizational interventions in combination has also not been studied.

Organizational efforts that target burnout are an essential step to enhance physician engagement and improve teamwork. Regulations related to documentation, adequate and meaningful use of EHRs, and workflow should be implemented. Insurance companies' requirements that physicians perform and document unnecessary elements that do not improve medical care should be eliminated. The National Institutes of Health should allocate funds to support research on clinician well-being and its impact on the care delivery system as well as strategies looking into improvement of work-life balance. Organizational policies that require physician maintenance of their certification must be accompanied by appropriate allocation of protected time for physicians to complete these tasks.

Healthcare institutions should recognize the potential effect of physician wellbeing on the quality of their performance. Well-being should be assessed as an institutional performance metric alongside measures such as cost, patient volume and patient satisfaction. This could help to allocate resources to work units in greatest need. Practice models proven to increase productivity and efficiency should be identified, adapted to fit the organization, adopted, and spread.

Physicians should also do their part. Regular self-evaluation should be considered a standard of optimal professionalism. Adequate sleep, family time, exercise, and attention to personal medical needs should be considered a minimal standard

\begin{tabular}{ll}
\hline Level & Proposed solutions \\
\hline Organizational effort & - Allocation of funding/support for research on physician well-being \\
- Optimize use of EHR & - Establish boundaries with insurance companies regarding required \\
documentation to minimize physician workload. Appropriate interpretation \\
of regulatory requirements \\
- The use of institutional metrics like cost, patient volumes, and patient \\
satisfaction to assess well-being \\
- Allocation of resources to work units in greatest need \\
- Leadership training \\
- Enhance self-awareness. Regular self-evaluation should be priority of the \\
core component of professionalism \\
- Ensure adequate sleep, exercise, and/or family time \\
- Prioritize goals \\
- Optimization of daily schedules; limit obligations to those required
\end{tabular}

Table 2.

Proposed solutions for physician burnout. 
for self-care. Building community at work and connections with colleagues has also been shown to reduce burnout and should be pursued [70].

Lastly, individual physicians should prioritize life goals. These goals should be realistic. Daily schedules should be optimized, limiting obligations and including protected time dedicated to self-care. The best way to do this is by learning effective and professional strategies to say "no." Individuals must continually evaluate their current condition, compare it with the ideal situation, and make the specific changes needed to approach the ideal state. When the status quo diverges from the ideal, change must be sought (Table 2 ).

\section{Conclusion}

Burnout, characterized by depersonalization, emotional exhaustion, and reduced personal accomplishment, has become a pandemic in healthcare. It has been associated with higher rates of depression among physicians, and its negative effect on overall work productivity and patient care is well established. Multiple factors contribute to physician burnout including work-related factors (work hours, call shifts, time spent on work from home, and the presence of work-home conflicts) as well as personal factors (sex, age, educational debt, relationship status, and presence of children). Higher rates of burnout have been reported among female physicians, and burnout is reported in both sexes as early as the first years of medical school. Burnout is also prevalent in both undergraduate and graduate medical education, and programs to mitigate burnout in these settings have been proposed. The consequences of physician burnout range from personal effects, such as increased rates of depression, substance abuse, sleep disturbances, and even suicides, to patient safety concerns, such as increased occurrence of medical errors, suboptimal patient care, and increased litigation. Physiciandirected interventions, such as mindfulness and cognitive behavioral techniques, target identification of burnout symptoms and development of personal coping strategies. Organization-directed interventions that involve workload changes, operation practices within the institution, increased level of leadership, and participation in decision-making among healthcare providers have also been proven to reduce the rates of burnout. Institutional efforts like the Energy Leadership Well-Being and Resiliency Program from University of Arizona and the ListenAct-Develop Model from Mayo Clinic and national strategies such as the Action Collaborative on Clinician Well-Being and Resilience network from the National Academy of Medicine have been launched to identify burnout drivers and symptoms and encourage timely adoption of changes to battle these drivers and achieve optimal patient care. Awareness of this problem among individual physicians and the healthcare community is imperative. Recognizing the drivers and personal triggers of burnout and applying timely interventions are the keys to mitigating this harmful syndrome.

\section{Conflict of interest}

The authors have no conflicts of interest to disclose. 


\section{Author details}

Bess Connors ${ }^{\dagger}$, Charlotte Horne ${ }^{\dagger}$, Valery Vilchez ${ }^{\dagger}$ and Sofya Asfaw*

Department of General Surgery, Cleveland Clinic Foundation, Cleveland, OH, USA

*Address all correspondence to: sofya.asfaw@gmail.com

${ }^{\dagger}$ These authors share equal contributions to this work.

\section{IntechOpen}

(C) 2019 The Author(s). Licensee IntechOpen. This chapter is distributed under the terms of the Creative Commons Attribution License (http://creativecommons.org/licenses/ by/3.0), which permits unrestricted use, distribution, and reproduction in any medium, provided the original work is properly cited. (cc) BY 


\section{References}

[1] Rotenstein LS, Torre M, Ramos MA, et al. Prevalence of burnout among physicians: A systematic review. Journal of the American Medical Association. 2018;320(11):1131-1150

[2] West CP, Dyrbye LN, Shanafelt TD. Physician Burnout: Contributors, consequences and solutions. Journal of Internal Medicine. 2018;283(6):516-529

[3] Shanafelt TD, Boone S, Tan L, et al. Burnout and satisfaction with work-life balance among US physicians relative to the general US population. Archives of Internal Medicine. 2012;172(18):1377-1385

[4] Dyrbye L, Thomas MR, Massie FS, et al. Burnout and suicidal ideation among US medical students. Annals of Internal Medicine. 2008;149(5):334-341

[5] Thomas NK. Resident burnout. Journal of the American Medical Association. 2004;292(23):2880-2889

[6] Balch CM, Shanafelt TD, Sloan JA, et al. Distress and career satisfaction among 14 surgical specialties, comparing academic and private settings. Annals of Surgery. 2011;254(4):558-568

[7] Dyrbye LN, Shanafelt TD, Balch CM, et al. Relationship between work-home conflicts and burnout among American surgeons: A comparison by sex. Archives of Surgery. 2011;146(2):211-217

[8] Shanafelt TD, Hasan O, Dyrbye LN, et al. Changes in burnout and satisfaction with work-life balance in physicians and the general US working population between 2011 and 2014. Mayo Clinic Proceedings. 2015;90(12):1600-1613

[9] Freudenberger HJ. Staff burnout. Journal of Social Issues. 1974;30:159-165
[10] Maslach C, Jacson SE, Leiter MP. Maslach Burnout Inventory Manual. 3rd ed. Palo Alto, CA: Consulting Psychologist Press; 1996

[11] DeCaporale-Ryan L, Sakran JV, Grant SB, et al. The undiagnosed pandemic: Burnout and depression within the surgical community. Current Problems in Surgery. 2017;54(9):453-502

[12] Bianchi R, Schonfeld IS, Laurent E. Burnout-depression overlap: A review. Clinical Psychology Review. 2015;36:28-41

[13] Schernhammer ES, Colditz GA. Suicide rates among physicians: A quantitative and gender assessment (meta-analysis). The American Journal of Psychiatry. 2004;161(12):2295-2302

[14] Andrew LB. Physician

Suicide: Overview, Depression in Physicians, Problems With Treating Physician Depression. [online] Emedicine.medscape.com. 2016 Available at: https://emedicine. medscape.com/article/806779-overview [Accessed 4 Mar. 2019]

[15] Downing NL, Bates DW, Longhurst CA. Physician burnout in the electronic health record era: Are we ignoring the real cause? Annals of Internal Medicine. 2018;169(1):50-51

[16] Shanafelt TD, Dyrbye LN, Sinsky C, et al. Relationship between clerical burden and characteristics of the electronic environment with physician burnout and professional satisfaction. Mayo Clinic Proceedings. 2016;91:836-848

[17] Shanafelt TD, West CP, Sloan JA, Novotny PJ, Poland GA, Menaker R, et al. Career fit and burnout among academic faculty. Archives of Internal Medicine. 2009;169(10):990-995

[18] Linn LS, Brook RH, Clark VA, Davies AR, Fink A, Kosecoff J. Physician 
and patient satisfaction as factors related to the organization of internal medicine group practices. Medical Care. 1985;23(10):1171-1178

[19] Tyssen R, Vaglum P, Grønvold NT, Ekeberg $\varnothing$. Factors in medical school that predict postgraduate mental health problems in need of treatment. A nationwide and longitudinal study. Medical Education. 2001;35(2):110-120

[20] Association of American Medical Colleges. FACTS: Total graduates by U.S. medical school and sex, 2002-2010. 2012. Available from: https://www. aamc.org/download/147312/data/ table29-gradsraceeth0210_test-web.pdf. pdf [Accessed: November 18, 2018]

[21] McMurray JE, Linzer M, Konrad TR, et al. The work lives of women physicians: Results from the physician work life study. Journal of General Internal Medicine. 2000;15:372-380

[22] Houle C, Harwood E, Watkins A, Baum KD. What women want from their physicians: A qualitative analysis. Journal of Women's Health. 2007;16:543-550

[23] Mast MS, Hall JA, Roter DL. Disentangling physician sex and physician communication style: Their effects on patient satisfaction in a virtual medical visit. Patient Education and Counseling. 2007;68:16-22

[24] Linzer M, Harwood E. Gendered expectations: Do they contribute to high burnout among female physicians. Journal of General Internal Medicine. 2018;33(6):963-965

[25] Saten SA, Holt DB, Jemp JD, Hemphill RR. Burnout in medical students: Examining the prevalence and associated factors. Southern Medical Journal. 2010;103(8):758-763

[26] IsHak W, Nikravesh R, Lederer S, et al. Burnout in medical students: A systematic review. The Clinical Teacher. 2013;10(4):242-245

[27] Dyrbye LN, Thomas MR, Huntington JL, et al. Personal life events and medical student burnout: A multicenter study. Academic Medicine. 2006;81(4):374-384

[28] Lebares CC, Guvva EV, Ascher NL, et al. Burnout and stress among US surgery residents: Psychological distress and resilience. Journal of the American College of Surgeons. 2018;226(1):80-90

[29] Shanafelt TD, Bradley KA, Wipf JE, Back AL. Burnout and self-reported patient care in an internal medicine residency program. Annals of Internal Medicine. 2002;136(5):358-367

[30] Purdy RR, Lemkau JP, Rafferty JP, Rudisill JR. Resident physicians in family practise: Who's burned out and who knows. Family Medicine. 1987;19(3):203-208

[31] Nyssen AS, Hansez I, Baele P, et al. Occupational stress and burnout in anaesthesia. British Journal of Anaesthesia. 2003;90(3):333-337

[32] Baldwin PJ, Dodd M, Wrate RW. Young doctors' health: How do working conditions affect attitudes, health and performance. Social Science $\&$ Medicine. 1997;45:35-40

[33] Erez A, Judge TA. Relationship of core self evaluations to goal setting, motivation and performance. The Journal of Applied Psychology. 2001;86(6):1270-1279

[34] Milam LA, Cohen GL, Mueller C, Salles A. The Relationship Between Self-Efficacy and Well-Being Among Surgical Residents. Journal of Surgical Education. 2019;76(2):321-328

[35] Bosk CL. Forgive and Remember: Managing Medical Failure. 2nd ed. 
Vol. 276. Chicago, IL: University of Chicago Press; 1979

[36] Luu S, Patel P, St-Martin L, et al. Waking up the next morning: Surgeons' emotional reactions to adverse events. Medical Education. 2012;46(12):1179-1188

[37] Lemaire JB, Wallace JE. Not all coping strategies are created equal: A mixed methods study exploring physicians' self report coping strategies. BMC Health Services Research. 2010;10(1):1

[38] Melamed S, Shirom A, Toker S, et al. Burnout and risk of cardiovascular disease: Evidence, possible causal paths, and promising research directions. Psychological Bulletin. 2006;132:327-353

[39] Shanafelt TD, Balch CM, Bechamps $\mathrm{G}$, et al. Burnout and medical errors among American surgeons. Annals of Surgery. 2010;251(6):995-1000

[40] Shanafelt TD, Mungo M, Schmitgen J, et al. Longitudinal study evaluating the association between physician burnout and changes in professional work effort. Mayo Clinic Proceedings. 2016;91(4):422-431

[41] Dewa CS, Loong D, Bonato S, et al. How does burnout affect physician productivity? A systematic literature review. BMC Health Services Research. 2014;14:325

[42] Bianchi R, Boffy C, Hingray C, Truchot D, Laurent E. Comparative symptomatology of burnout and depression. Journal of Health Psychology. 2013;18(6):782-787

[43] Glass DC, McKnight JD, Valdimarsdottir H. Depression, burnout, and perceptions of control in hospital nurses. Journal of Consulting and Clinical Psychology. 1993;61(1):147

[44] Becker JL, Milad MP, Klock SC. Burnout, depression, and career satisfaction: Cross-sectional study of obstetrics and gynecology residents. American Journal of Obstetrics and Gynecology. 2006;195(5):1444-1449

[45] Oreskovich MR, Kaups KL, Balch CM, Hanks JB, Satele D, Sloan J, et al. Prevalence of alcohol use disorders among American surgeons. Archives of Surgery. 2012;147(2):168-174

[46] Davis M, Detre T, Ford DE, Hansbrough W, Hendin H, Laszlo J, et al. Confronting depression and suicide in physicians: $A$ consensus statement. Journal of the American Medical Association. 2003;289(23):3161-3166

[47] van der Heijden F, Dillingh G, Bakker A, Prins J. Suicidal thoughts among medical residents with burnout. Archives of Suicide Research. 2008;12(4):344-346

[48] Donaldson MS, Corrigan JM, Kohn LT, editors. To Err is Human: Building a Safer Health System. Washington, D.C.: National Academy Press; 2000

[49] Seys D, Wu AW, Gerven EV, Vleugels A, Euwema M, Panella $\mathrm{M}$, et al. Health care professionals as second victims after adverse events: A systematic review. Evaluation \& The Health Professions. 2013;36(2):135-162

[50] West CP, Huschka MM, Novotny PJ, Sloan JA, Kolars JC, Habermann TM, et al. Association of perceived medical errors with resident distress and empathy: A prospective longitudinal study. Journal of the American Medical Association. 2006;296(9):1071-1078

[51] Hayward RA, Hofer TP. Estimating hospital deaths due to medical errors: Preventability is in the eye of the reviewer. Journal of the American Medical Association. 2001;286(4):415-420 
[52] Brennan TA, Leape LL, Laird NM, Hebert L, Localio AR, Lawthers AG, et al. Incidence of adverse events and negligence in hospitalized patients: results of the Harvard Medical Practice Study I. New England Journal of Medicine. 1991;324(6):370-376

[53] Hayward RA, McMahon LF, Bernard AM. Evaluating the care of general medicine inpatients: How good is implicit review? Annals of Internal Medicine. 1993;118(7):550-556

[54] Williams ES, Manwell LB, Konrad TR, Linzer M. The relationship of organizational culture, stress, satisfaction, and burnout with physician-reported error and suboptimal patient care: Results from the MEMO study. Health Care Management Review. 2007;32(3):203-212

[55] Firth-Cozens J, Greenhalgh J. Doctors' perceptions of the links between stress and lowered clinical care. Social Science \& Medicine. 1997;44(7):1017-1022

[56] Halbesleben JR, Rathert C. Linking physician burnout and patient outcomes: Exploring the dyadic relationship between physicians and patients. Health Care Management Review. 2008;33(1):29-39

[57] Balch CM, Oreskovich MR, Dyrbye LN, Colaiano JM, Satele DV, Sloan JA, et al. Personal consequences of malpractice lawsuits on American surgeons. Journal of the American College of Surgeons. 2011;213(5):657-667

[58] DiMatteo MR, Sherbourne CD, Hays RD, Ordway L, Kravitz RL, McGlynn EA, et al. Physicians' characteristics influence patients' adherence to medical treatment: Results from the Medical Outcomes Study. Health Psychology. 1993;12(2):93

[59] Kushnir T, Greenberg D, Madjar N, Hadari I, Yermiahu Y, Bachner YG. Is burnout associated with referral rates among primary care physicians in community clinics? Family Practice. 2013;31(1):44-50

[60] West CP, Dyrbye LN, Erwin PJ, et al. Interventions to prevent and reduce physician burnout: A systematic review and meta-analysis. Lancet. 2016;388:2272-2281

[61] Shanafelt TD, Balch CM, Dyrbye LN, et al. Special report: Suicidal ideation among American surgeons. Archives of Surgery. 2011;146:54-62

[62] Panaglioti M et al. Controlled interventions to reduce burnout in physicians. JAMA Internal Medicine. 2017;177(2):195-205

[63] Hutter MM, Kellogg KC, Ferguson CM, et al. The impact of the 80-hour resident workweek on surgical residents and attending surgeons. Annals of Surgery. 2006;243:864-875

[64] Salles A, Nandagopal K, Walton G. Belonging: A simple, brief intervention decreases burnout. Journal of the American College of Surgeons. 2013;217:S116

[65] Riall T, Teiman J, Chang M, et al. Maintaining the fire but avoiding burnout: Implementation and evaluation of a resident well-being program. Journal of the American College of Surgeons. 2018;4:369-379

[66] Swensen S, Kabcenell A, Shanafelt T. Physician-organization collaboration reduces physician burnout and promotes engagement: The Mayo Clinic experience. Journal of Healthcare Management. 2016;61:105-127

[67] Berry LL, Seltman KD. The enduring culture of Mayo Clinic. Mayo Clinic Proceedings. 2014;89(2):144-147

[68] Dilling JA, Swensen SJ. Accelerating the use of best practices: The Mayo Clinic model of diffusion. The Joint 
Commission Journal on Quality and

Patient Safety. 2013;39(4):167-176

[69] National Academy of Medicine.

Action collaborative on clinician well-

being and Resilience. 2018. Available

from: https://nam.edu/initiatives/

clinician-resilience-and-well-being

[70] Shanafelt TD, Noseworthy JH.

Executive leadership and physician

well-being: Nine organizational

strategies to promote engagement and

reduce burnout. Mayo Clin Proceedings.

2017;92(1):129-146 


\title{
Wellness in Residency: A Paradigm Shift
}

\author{
Roderick M. Quiros and Elspeth Black
}

\begin{abstract}
There has been a cultural shift in the state of residency training over the past two decades. While the traditional view of trainees heavily emphasized the service component of residency, training programs are gaining an increasing awareness of the trainees' well-being as crucial to their functioning, the success of the training program, and ultimately, to the care of patients. To this end, work-hour limitations have been imposed universally. Additionally, some programs have established interventions that allow residents to lead balanced lives with emphasis on time away from work, sleep, and outside activities. A paradigm shift recognizing the importance of wellness in residency may reduce the risk of physician burnout in the long term.
\end{abstract}

Keywords: residency, training, wellness, well-being, burnout

\section{Introduction}

The notion of the physician as an agent by which health is maintained or restored is an archetypal one. While it is a noble image, it does not address the possibility that the physician's own health may be compromised, even as he or she works in the service of patients. Physicians in training are at risk for depression; continued stressors after completion of residency have elevated the risk of suicide in physicians compared to the general population. Furthermore, the rate of death by suicide is approximately $70 \%$ higher for male physicians compared to men in the general population, and between 250 and $400 \%$ higher for female physicians than women in the general population [1]. These numbers are startling and should initiate widespread changes in how we train our future physicians, but they are far from new data. A brief literature review will reveal similar statistics published in the 2000s, the 1990s, the 1980s, and nearly every decade before that for over a century [2].

It has also been demonstrated that there is a preponderance of burnout in medicine that is greater than that of the general US working population. Nearly half of all US physicians report at least one symptom of burnout, and physicians were more likely than their non-physician counterparts to be dissatisfied with work-life balance (approx. 40 vs. 23\%) [3]. Especially pertinent in these times of the US physician shortage are the statistics demonstrating that those on the front lines of primary care are more at risk. Even after adjusting for demographics and such frequently blamed characteristics such as hours worked per week and call schedule, physicians practicing emergency medicine, general internal medicine, and family medicine remained at higher risk of burnout [3]. It is thought that the US loses approximately 1 doctor every day to suicide, and assuming the average family 
physician patient panel is 1500 patients (which is a low estimate), the extrapolated public health crisis is that over $1 / 2$ million patients in the US per year are losing their physicians due to suicide $[2,4,5]$. This number does not include doctors lost due to insurance changes, natural death, moving, and the litany of other reasons that patients lose continuity care with their primary care providers.

The US healthcare system could benefit from changes across the board, but the specific malady of physician wellness is treatable. The current culture of medical training promotes a strong, resilient-to-a-fault façade that contributes to a perceived negative stigma toward receiving help for burnout and depression $[6,7]$. By shifting the way medical students and residents are recruited, mentored, and trained, the culture should gradually shift to one of encouragement rather than one of punition. Making this change requires an understanding of the journey to becoming a physician.

\subsection{The risk of burnout is inherent from the start}

The first step in becoming a physician is acceptance into medical school. This is achieved by being an elite, well-rounded student with excellent exam scores. The selection process tends to favor highly motivated students who are at the top of their class [7]. Interestingly, while students entering medical schools are no different from their non-medical peers in terms of their mental health, their overall mental health state starts to decline soon after they begin their medical studies [8]. Once in medical school, the student experiences the so-called firehose of information that composes the first 2 years of medical school. While attempting to learn this extremely broad and detailed material, the competition continues; one is expected to excel at coursework while also getting involved in research and interest groups for the specialties for which one may apply. In the 3rd and 4th years of medical school, the students are thrust into the clinical hierarchies of the hospital education system. It is expected that the 3 rd year student will come in early and leave late while trying to learn as much as possible and trying to impress their senior staff who may eventually write their recommendation letters. The 4 th year medical student may gain some comfort as he or she develops some mastery in the workflow of their training and after matching into the residency of their choice. Comfort may be short lived as the young doctor catches sight of the true summit as they begin their intern year, and the road seems to be stretching limitlessly ahead yet again.

An interesting phenomenon that begins to occur in medical school, but that is not exclusive to the field of medicine, is the imposter syndrome (IS). This is a state characterized by feelings of self-doubt and fear of being outed as an intellectual fraud, despite direct evidence to the contrary. Affected individuals experience an inability to feel accomplished in their field and that they are lacking the competency that others perceive them to have [7]. Studies show that approximately $25 \%$ of male medical students and nearly $50 \%$ of female medical students experience IS. The experience of IS is significantly associated with burnout, which leads one to ponder what influences in the medical education system are leading so strongly to both burnout and IS. Some suggest it is the milieu of competition in the setting of shamebased learning and the overall teaching style that contribute to their growth [7].

Upon graduation from medical school and at the start of residency, growth and learning again occur logarithmically. While the vast majority of residents complete their respective programs, the intensity and duration of training can take a toll on the resident's well-being. Some programs in particular may seem to struggle with resident wellness, and seeds of discontent quickly spread among the trainees. This in turn can lead to the training institution being labeled a "malignant program" among the network of residents. A malignant residency program is one where the residents feel 
overworked and undervalued, shifts are long, days off are rare, duty hour rules are constantly flouted, residents feel demeaned and disrespected, faculty are disinterested and/or belligerent, and the light at the end of the tunnel frequently feels very far away. One can logically conclude that at these programs, one would find the ultimate environment for burnout. Research in this area is strongly lacking, most likely due to the lack of reporting. There is a culture of fear and silence in these programs, as the resident who reports their own program perceives themselves to be knowingly putting their own career at risk. Thus, the term "malignant program" is found everywhere in the blogosphere but is exceptionally rare in the medical literature [9-11].

With a clear link between physician burnout and the public health crisis of the consequence of that burnout, it is important that we can measure the burnout severity and make appropriate recommendations to those identified at highest risk. Before we can measure burnout, we must first understand what it is. As defined by Christina Maslach, burnout is a syndrome characterized by a loss of enthusiasm for work (emotional exhaustion), feelings of cynicism (depersonalization), and reduced personal accomplishment [12]. There has been a lot of focus both in the popular press as well as in medical literature about monitoring, defending against, and eradicating burnout. There has been much less focus on the defining of, education in, and promotion of physician wellness [13]. This makes for a conundrum where students may know what to avoid but not necessarily be equipped to navigate between the pitfalls. Educators may promote behaviors like "self-care" and encourage students to get enough sleep without necessarily laying out a plan by which their students could accomplish these goals.

Eckleberry-Hunt and colleagues describe the promotion of wellness through changing the culture of medicine. They defined wellness as "a dynamic and ongoing process involving self-awareness and health choices resulting in a successful, balanced lifestyle" [13]. Tenets of wellness need to be woven into the fabric of medical education, just as clearly emphasized as those of professionalism and patient safety. The means for incorporating wellness into residency training may lie in the establishment of a curriculum that objectively measures resident wellness and identifies and addresses potential burnout. Ultimately, such a curriculum would contribute to career longevity long after completion of residency.

\section{Physician training: the traditional model}

While the concept of a physician in training has always included both a service component (to the patient) as well as an educational component (for the trainee rendering care), the historical model emphasized the service component to a higher degree. The expectation was that under the guidance and instruction of a more senior physician, the trainee would learn by doing; working in the trenches, so to speak, would impart on-the-job experience. Indeed, this "master-apprentice" model was the norm until the late nineteenth century, with trainees often starting in their teenage years. The pupil was expected to learn by directly observing, then imitating the master's technical and clinical skills. While seemingly personal in that the relationship between mentor and mentee was an individualized, tailored one, the disadvantage to this was that there were no standardized guidelines as to what knowledge was to be imparted or for how long training was to last.

In the 1890s, Sir William Osler introduced bedside rounds with students at the Johns Hopkins School of Medicine. This was a break from the standard practice, as direct patient contact was thought to be beyond the skills of students. Soon after, William Halstead, Osler's surgical counterpart, took over the department of surgery at Johns Hopkins and decided to incorporate principles of basic science into the clinical 
curriculum. The Halstead model for training emphasized recurrent opportunities for trainees to take care of patients under supervision of a skilled teacher, married to the need for the trainee to understand the scientific basis of disease and the need for the trainee to acquire skills in management of increasing complexity with graded responsibility and ultimately, independence with each advancing year [14].

While the length of training and the general graded curriculum was established both in surgical and medical specialties, the balance between service and education was weighted toward the former. Trainees lived up to the name "residents" who were expected to be ever present in hospital and available for continuous patient care. This was the expectation for the last century, and while the system did have its advantages, namely, nearly continuous immersion in the clinical arena (and presumably continuous learning), it did have its share of problems as well in that it tended to dehumanize the trainee to the detriment of caregiver and patient alike. The resident was expected to work tirelessly, often at the expense of his/her personal well-being and health. Physical and mental fatigue were understood and accepted as part of the job and even an expected part of the training as it imparted a toughness to the trainee that would serve them well upon completion of their training.

\subsection{Culture change: the impact of the Libby Zion Law}

Libby Zion was a freshman college student who, in March of 1984, was admitted to a New York City hospital by her family physician for workup of flu-like symptoms which she had been experiencing for several days. She was assigned to the care of two resident physicians. The two residents could not pinpoint the cause of Libby's symptoms and ultimately prescribed meperidine to help manage the jerking motions that Libby displayed on admission. One of the residents also ordered restraints be placed on Libby and prescribed Haldol to help control agitation. At the time, both residents were covering several other patients in the hospital.

Libby ultimately fell asleep, but on the following morning, she was febrile to $107^{\circ} \mathrm{F}$. She shortly had a cardiac arrest and could not be revived. Ultimately, Libby's death was attributed to the interaction of an antidepressant, phenelzine, which she had been taking prior to admission to the hospital, with the meperidine given to her by the residents. The combination led to development of serotonin syndrome, which is associated with increased agitation and fever. The fever progressed to the point of causing cardiac dysfunction and arrest.

Libby's parents were convinced that her death was due to staffing problems at the hospital. Libby's father Sidney took issue with the long hours, and residents were working at the time of her death as he believed that residents working a 36-hour shift were incapable of making medical judgment calls. The residents in this case had prescribed a medication that ultimately led to an untoward effect by interaction with the patient's own medication. They also ordered restraints for her when she progressively became agitated. Sidney attributed these errors in part due to the residents working prolonged shifts and making erroneous judgments due to fatigue [15]. These events culminated in a lawsuit against the residents.

The case ultimately became a high-profile legal battle with multiple charges brought against the residents involved. Ultimately, after several years of litigation, charges against the residents were dropped. Additionally, the court completely cleared the records of the two residents of findings that they had provided inadequate care [16].

The case put the spotlight on the effect of resident work hours on patient care and led to the formation of a panel of experts headed by Dr. Bertrand Bell from the Albert Einstein College of Medicine, another New York institution. This committee, which became known as the Bell commission, evaluated the training of doctors in 
New York State and ultimately made a series of recommendations based on their findings. In 1989, New York State adopted the Commission's recommendations that residents could not work more than 80 hours a week or for more than 24 consecutive hours. In 2003, the Accreditation Council for Graduate Medical and Education (ACGME) adopted similar regulations for all accredited medical training institutions, regardless of specialty [17].

\subsection{The ACGME and the 80-hour work week}

Before the adoption of such regulations, it was understood that physicians in training would commonly work between 80 and 100 hours per week, sometimes more, as a matter of routine. While a portion of this time was involved in direct patient care, some of those hours involved other tasks indirectly related to patient care such as paperwork. While the Libby Zion case was not directly about prolonged resident working hours, the aftermath led to scrutiny on the role of prolonged hours on residents and the patients for which they were responsible and the resulting Bell Commission's recommendations. Residents on call are expected to be available at all times during their 24 hour shifts while in hospital, and, if particularly busy, may be awake for the full 24 hours of the shift. A survey of 1st and 2 nd year residents revealed that $66 \%$ of respondents got 6 hours or less of sleep per night, while $20 \%$ got 5 hours or less of sleep per night [18]. Presumably, this sleep schedule applies to days when the residents are not on call/or in hospital.

The expectation that physicians in training were to be near constantly available was in part based on the assumption that residency positions are limited and highly desired; residents therefore willingly subject themselves to such a work schedule. Getting through residency, particularly the ones with highly demanding schedules and workloads, was a sign of toughness and a badge of honor. Additionally, the continuity of care of the patient, which is conceptually sound, can lead to prolonged hours, and a physician accepting a patient early in their shift may be compelled to follow that patient's care well beyond the end of their shift in order to follow through with any patient-related issues [19].

The case against the "old school" schedule present before the start of the 80-hour workweek in 2003 is based in part on the decline of physician performance from mental and physical fatigue resulting from sleep deprivation. Multiple studies have shown that residents who are chronically sleep-deprived are acutely at higher risk for personal harm such as motor vehicle accidents, as well as work-related injuries like sharps injuries or needlesticks. Over the long term, lack of sleep can also put physicians both in training and beyond at higher risk for cardiovascular disease, being overweight, or diabetes [20].

Resident sleep deprivation adversely affects trainees both mentally and physically, which may translate into poorer patient outcomes. In a meta-analysis of 959 physicians, a 24 to 30-hour continued state of being awake without sleep decreased physicians' overall performance by almost 1 standard deviation and clinical performance by more than 1.5 standard deviations [21]. A web-based survey by Barger et al. showed that interns committed significantly more fatigue-related medical errors culminating in adverse patient outcomes on months with five or more overnight calls than with months with no extended shifts [22]. Another study showed the effects of sleep deprivation on residents' technical skills; surgical residents who had been awake the previous night made $20 \%$ more errors and took $14 \%$ longer to complete a simulated laparoscopic task than their colleagues who had slept well the previous night [23]. A study found that after 24 hours of sustained wakefulness, hand-eye coordination decreased to a level equal to the performance observed at a blood alcohol concentration of roughly $0.10 \%$ [24]. 
In 2003, under pressure from OSHA (the Occupational Safety and Health Administration) and other regulatory bodies, the ACGME responded to these and other data by crafting mandatory duty hour requirements for all ACGME-accredited training programs. First, the number of hours that trainees would be capped is at 80 hours per week, averaged over 4 weeks, and inclusive of all in-house call activities. Residents were to receive a minimum 10 -hour rest period between duty periods and after in-house call. Call itself could not exceed a 24 hour limit. However, while residents could not receive new patients after 24 hours of continuous duty, there are allowable provisions of up to 6 hours for continuity of care or for education. Residents were to have 1 day a week off, averaged over 4 weeks. Finally, there has to be an in-house call no more than once every 3 nights, averaged over 4 weeks.

Early data after the institution of the 80 -hour workweek seemed to show a benefit to the new guidelines. In 2004, one study that found reducing sleep deprivation significantly reduced errors in intensive care units [25]. This study from The Brigham and Women's Hospital study was a prospective, randomized study comparing the rates of serious medical errors made by interns while they were working according to a traditional schedule with extended ( 24 hours or more) work shifts every other shift (an "every third night" call schedule) and while they were working according to an experimental schedule that eliminated extended work shifts and reduced the number of hours worked per week. Adverse incidents were identified by means of a multidisciplinary approach that included direct, continuous observation where two physicians blinded to the interns' schedule assignments independently rated each incident. During a total of 2203 patient days on 634 admissions, interns made $35.9 \%$ more serious medical errors during the traditional schedule than during the experimental schedule. The rate of serious errors on the critical care units was $22.0 \%$ higher during the traditional than during the experimental schedule. Interns made $20.8 \%$ more serious medication errors during the traditional than during the experimental schedule. Interns also made 5.6 times as many serious diagnostic errors during the traditional as during the experimental schedule. The authors concluded that interns made substantially more serious medical errors when they worked frequent shifts of 24 hours or more. Based on their data, the authors recommended eliminating extended work shifts and reducing the number of weekly hours in order to reduce medical errors in the intensive care unit.

In the years after the ACGME adopted the 80-hour workweek, these restrictions were again scrutinized. In 2008, the common duty hour requirements instituted in 2003 had been in effect for five years, and the ACGME was prepared to refine their recommendations. Ongoing investigation of interns in particular resulted in a further reduction in work hours for that subset of trainees, so that in 2011, the number of continuous hours an intern could work was reduced to 16 . This rule affected more senior residents and some faculty who, with interns working fewer hours, were forced to provide more coverage. Not surprisingly, senior resident and faculty satisfaction concurrently declined after this rule was put in place.

The debate on schedule restrictions for residents is ongoing. Several questions remain unanswered, as more recent data on the beneficial effects of hour reduction are mixed. Are 80 hours a week appropriate or even too much? Other countries besides the US place limits ranging from 37 hours per week in Denmark to 48 in the European Union [26]. Are residents missing valuable experiences monitoring the course of illness with their patients? There is an old saying in surgical training that the problem with every-other-night call is that the resident misses half of all the interesting cases in the hospital; clearly this sentiment originated well before 2003. While the average person may agree that 8 hours of sleep per night may be ideal, the corollary question as it applies to residency training is, "What amount of time for rest and recovery does a person need between shifts?" 


\section{Resident wellness: it is more than just about hours worked}

While the institution of restrictions on duty hours was thought to result in safer, higher quality medicine as well as improvements in resident quality of life, there have been unintended negative consequences to reduced working hours. Bolster and Rourke reviewed papers published between 2010 and 2013, specifically on interventions made as a result of duty hour restrictions (such as night float systems, shortened shifts, and protected time for sleep) and measured the effects of the interventions on patient care, resident well-being, and resident education [27]. They found that frequently, the studies as a whole concluded that the restrictions had no impact on patient care $(50 \%)$ or resident wellness $(47 \%)$, and actually had a negative impact on resident education (64\%). The night float system was the most universal way of implementing duty hour restrictions, though ironically it was actually associated with the highest proportion of unfavorable findings. The authors concluded that a focus on duty hours alone did not result in consistent improvements in patient care or resident well-being and that the added duty hour restrictions implemented in 2011 appear to have had an unintended negative impact specifically on resident education. They concluded that residents missed educational opportunities, including requisite teaching conferences, on their required time out of the hospital.

Another systematic review among surgical programs showed an inconsistent effect of restricted resident duty hours on resident wellness and a negative impact on patient outcomes and performance on certification exams [28]. This study included papers written between 1980 and 2013. Articles that examined mortality data were combined in a random-effects meta-analysis to evaluate the impact of resident duty hours on patient mortality. There was no overall improvement in patient outcomes as a result of reduced resident hours; however, some studies suggest increased complication rates in high-acuity patients. There was no improvement in education related to duty hour restrictions, and surprisingly, performance on certification examinations actually declined in some specialties. Additionally, while there were collective improvements in resident wellness after the institution of the 80-hour workweek in 2003, there was little improvement and even some negative effects on wellness after the 16-hour duty maximums were implemented in 2011.

One of the concerns for training programs after the institution of resident duty hour restrictions is that some residents may adopt a "shift mentality" where one simply clocks in and out of work. This tendency challenges notion of physician professionalism where the trainee sees the case through to the end and maintains continuity of care. Training with the shift mentality does not reflect life after residency when the 80-hour restriction no longer applies. Part of the solution may be not just a reduction in duty hours but allowing flexibility in managing resident schedules in a way that patient care, resident wellness, and education are least adversely affected. The Flexibility in Duty Hour Requirements for Surgical Trainees (FIRST) Trial involved 117 American general surgical residency programs and was conducted to test whether surgical patient outcomes under flexible, less-restrictive duty hour policies would be non-inferior to those under standard ACGME policies [29]. Programs were randomized to current ACGME duty hour policies or more flexible policies that waived rules on shift lengths and time off between shifts. Outcomes included the 30-day rate of postoperative death or serious complications (primary outcome), other postoperative complications, and resident perceptions and satisfaction regarding their well-being, education, and patient care. The authors reported that flexible, less-restrictive duty hour policies were in fact not associated with an increased rate of death or serious complications $(9.1 \%$ in the flexible-policy group and $9.0 \%$ in the standard-policy group) or of any secondary postoperative 
outcomes studied. Among the 4330 residents in the study, those in programs assigned to flexible policies did not report significantly greater dissatisfaction with overall education quality (11.0\% in the flexible-policy group and $10.7 \%$ in the standard-policy group) or well-being (14.9 and $12.0 \%$, respectively). Residents under flexible policies were less likely to perceive negative effects of duty hour policies on multiple aspects of patient safety, continuity of care, professionalism, and resident education, though they were more likely to perceive negative effects on personal activities compared to those in the standard-policy group. There were no significant differences between study groups in resident-reported perception of the effect of fatigue on personal or patient safety. Residents in the flexible-policy group were less likely than those in the standard-policy group to report leaving during an operation (7.0 vs. $13.2 \%$ ) or handing off active patient issues ( 32.0 vs. $46.3 \%$ ). The authors concluded that less-restrictive duty hour policies for residents were equivocal regarding patient outcomes and lacked significant difference in residents' satisfaction with overall well-being and education quality, when compared to standard duty hour policies.

\section{Reactive to proactive: promoting wellness}

The reduction in resident work hours may be construed as a passive intervention in that resident well-being has been thought to benefit from time simply away from work. As such, the changes made by the ACGME were largely reactive to the data from the 1980s to 1990s showing adverse effects for unlimited work hours on both patients and residents. Over the last decade however, the concept of physician burnout has gained more prominence. This is an issue that undoubtedly can affect resident physicians but which can affect the physician well beyond residency into practice. Pursuing physician wellness has therefore become a more proactive endeavor thought to extend beyond simply cutting hours worked, since the 80 -hour restrictions do not apply in post-residency training.

In an analysis done at the Mayo Clinic, 7288 physicians were surveyed on their quality of life and job satisfaction [3]. The data revealed that $46 \%$ of respondents reported at least one burnout symptom. The report indicates that physicians both as a group and relative to other highly educated individuals working long hours suffer high levels of emotional exhaustion and struggle to find a satisfying work-life balance.

Unlike depression, which affects a person's life at home as well as at work, burnout generally refers to a constellation of symptoms specific to the workplace. Symptoms include depersonalization (a tendency to perceive and treat people like objects), a loss of a sense of personal accomplishment, and mental and emotional fatigue. By its nature, medicine as a profession attracts people who are drawn to service, even if it means self-sacrifice. Most physicians are inclined to do what is necessary to take care of patients, which in the long term can lead to fatigue and burnout. The Mayo clinic report showed that burnout was not limited to any one particular specialty, but that notable differences in burnout were observed, with the highest rates among physicians at the front line of care access (family medicine, general internal medicine, and emergency medicine). Despite this report, it must be emphasized that burnout was seen across all specialties to a varying degree.

The present generation of trainees, as well as senior staff responsible for their training, is being exposed to the concept of wellness more and more as it becomes integrated into the training culture and curriculum. The idea of wellness in residency, if not universal at present, is more commonly becoming a core element in training. There is some understanding that the best way to take care of one's patients is to take care of one's self. 


\subsection{Wellness in training and beyond}

In 2015, the ACGME formed a committee to comprise of 29 residents and fellows of various specialties to study resident wellness in the present state of training, propose the ideal learning environment to promote wellness, and determine how to bridge the differences between the two [30]. The council concluded that policies in training programs should increase awareness of the stress of residency, destigmatize the diagnosis of depression in trainees, develop ways to identify and treat depression confidentially to encourage trainees to get help, enhance mentoring by senior peers, promote a supportive culture, and encourage continuing study to deepen understanding of wellness. To this end, several residency training programs across the US have incorporated various resources to promote wellness. Generically, these include burnout prevention by formally emphasizing healthy eating, exercise, and mindfulness. Identifying signs and symptoms of burnout are stressed, as are resources to help manage them, such as mental health services. They also include scheduled recreational events with colleagues away from work, as well as the institution of a mentoring program with more senior residents or faculty. Some programs help with financial planning to address stress associated with monetary issues.

Social support, both professionally and personally, is a major pillar in preventing and combating burnout [31]. While conflicts between professional demands and home and family life may be a stressor contributing to burnout, and that married women in particular may be more affected by these conflicts, some data show that marriage is likely to reduce the risk overall $[32,33]$. Balch and colleagues reviewed a number of studies on the causes of burnout in physician training and beyond in an attempt to increase awareness of burnout and help identify ways to curb risk starting in residency (Table 1) [34]. In a follow-up study, Balch and colleagues sent out a cross-sectional survey to members of the American College of Surgeons [35]. The survey included questions about physicians' own health habits, routine medical care practices, personal wellness strategies, and assessments of burnout. From data collected from 7197 respondents, the most effective behaviors associated with lower burnout risk were incorporating wellness strategies that include finding meaning at work, focusing on what is important in life, maintaining a positive outlook and attitude toward professional life, and understanding that work-life balance may decrease risk of burnout and improve quality of life. More specific, tangible findings included increasing weekly exercise training to levels recommended by the

- Length of training and delayed gratification

- Limited control over the provision of medical services

- Long working hours and enormous workloads

- Imbalance between career and family

- Feeling isolated or loss of time to connect with colleagues

- Financial issues (salary, budgets, managed care, etc.)

- Grief and guilt about patient death of unsatisfactory outcome

- Insufficient protected research time and funding

- Sex- and age-related issues

- Inefficient and/or hostile workplace environment

- Setting unrealistic goals or having them imposed on oneself

Table 1.

Partial list of contributing causes to physician burnout (Originally Table 2 from Balch et al. [34]). 
Center for Disease Control, undergoing annual visits to one's primary care provider, and undergoing age-appropriate preventative testing, though the authors acknowledge that is uncertain if these measures are characteristics of surgeons who place a greater emphasis on self-care or if they are concrete ways to reduce burnout.

Paradoxically, burnout may mostly affect those physicians who are dedicated to their patients to the point of being "generous to a fault." The importance of empathy in medicine cannot be overstated, yet there is some question as to the actual relationship between empathy and burnout. Zenasni and colleagues presented possible ideas relating the two [36]. First, burnout is an "empathy killer"; when physicians tend toward burnout, they also tend to withdraw from relationships with patients as a defense mechanism to restore psychological balance. The authors also propose that empathy may create burnout as empathy at high levels predispose to "compassion fatigue" which can progress to burnout. The final hypothesis they present is that empathy can prevent burnout. The empathic physician has an awareness of negative emotions, requiring self-reflection and the need to be able to accept negative feedback, both of which are skills protective against burnout. More recently, Wilkinson and colleagues preformed a systematic review to clarify the relationship between empathy and burnout [37]. Their meta-analysis consisted of 10 studies, 8 of which were suggestive of a negative relationship between burnout and empathy; 1 study showed a positive relationship between the two; and lastly, 1 had both positive and negative correlations between burnout and empathy. While the majority of studies pointed toward a negative association between burnout and empathy, the authors concluded that additional research to establish causality was needed since all the included studies were cross-sectional.

\section{Conclusion}

Residency is both a demanding and rewarding experience for those entering the medical field. It is the training ground where physician education is intertwined with service to the patient and where a balance between formation of a sound and well-prepared clinician and a mentally and emotionally fit person as caregiver is struck. The last three decades have seen a shift from residents as a large service component in the healthcare system to persons whose well-being affects themselves, their colleagues, and their patients. Initiation of wellness training begins in residency, but principles espoused in such programs should be applied well beyond the end of training into professional life. In the following chapter, we will review the Maslach Burnout Inventory and the Utrecht Work Engagement Scale as objective means of measuring physician burnout. As a practical application, we will discuss a resident wellness curriculum in our own institution designed to recognize and prevent burnout and to promote physician well-being.

\section{Conflict of interest}

The authors of this work have no conflicts of interest to declare. 


\section{Author details}

Roderick M. Quiros ${ }^{1 *}$ and Elspeth Black ${ }^{2}$

1 St. Luke's University Health Network Department of Surgery, Bethlehem, PA, USA

2 St. Luke's University Health Network Department of Family Practice, Bethlehem, PA, USA

*Address all correspondence to: roderick.quiros@sluhn.org

\section{IntechOpen}

(C) 2018 The Author(s). Licensee IntechOpen. This chapter is distributed under the terms of the Creative Commons Attribution License (http://creativecommons.org/licenses/ by/3.0), which permits unrestricted use, distribution, and reproduction in any medium, provided the original work is properly cited. (cc) BY 


\section{References}

[1] Hampton T. Experts Address Risk of Physician Suicide. Journal of the American Medical Association. 2005;294(10):1189-1191

[2] Andrew L. Physician Suicide [Internet]. 2018. Available from: https://emedicine.medscape.com/ article/806779-overview

[3] Shanafelt T, Boone S, Tan L, Dyrbye L, Sotile W, Satele D, et al. Burnout and satisfaction with worklife balance among US physicians relative to the general US population. Archives of Internal Medicine. 2012;172(18):1377-1385

[4] Murray M, Davies M, Boushon B. Panel size: How many patients can one doctor manage? Family Practice Management. 2007;14(4):44-51

[5] Laff M. Patient Panel Size Uncertainty Complicates Workforce Projections [Internet]. Available from: https://www.aafp.org/news/practiceprofessional-issues/20160720rgcpanelsize.html

[6] Dyrbye L, Eacker A, Durning S, Brazeau C, Moutier C, Massie F, et al. The impact of stigma and personal experiences on the help-seeking behaviors of medical students with burnout. Academic Medicine. 2015;90(7):961-969

[7] Villwock J, Sobin L, Koester L, Harris T. Imposter syndrome and burnout among american medical students: A pilot study. International Journal of Medical Education. 2016;7:364-369

[8] Slavin S, Schindler D, Chibnall J. Medical student mental health 3.0: Improving student wellness through curricular changes. Academic Medicine. 2014;89(4):573-577
[9] Available from: https://www. kevinmd.com/blog/2015/01/tragicphysician-story-match-doesnt-wanthear.html

[10] Available from: https://www. kevinmd.com/blog/2015/02/rankingresidency-match-red-flags-watch.html

[11] Williams D. Cracking the code on the "Hidden Curriculum" in the medical education pipeline and its contribution to attrition. Journal of Unschooling and Alternative Learning. 2016;10 (19):82-83

[12] Maslach C, Jackson S, Leiter M, Schaufeli W, Schwab R. Maslach Burnout Inventory. Palo Alto, CA: Consulting Psychologists Press; 1986. pp. 3463-3464

[13] Eckleberry-Hunt J, Van Dyke A, Lick D, Tucciarone J. Changing the conversation from burnout to wellness: Physician well-being in residency training programs. Journal of Graduate Medical Education. 2009;1(2):225-230

[14] Grillo HC. To impart this art: The development of graduate surgical education in the united states. Surgery. 1999;125(1):1-14

[15] Lerner B. A Case That shook medicine: How one man's rage over his daughter's death sped reform of doctor training. The Washington Post, November 28, 2006

[16] Sack K. Appeals Court Clears Doctors Who Were Censured in the Libby Zion Case. The New York Times, November 1, 1991

[17] Philibert I, Friedmann P, Williams W. New requirements for resident duty hours. Journal of the American Medical Association. 2002;288(9):1112-1114 
[18] DeWitt C, Daugherty SR. Sleep deprivation and fatigue in residency training: Results of a national survey of first- and second-year residents.

Sleep. 2004;27(2):217-223

[19] Fargen K, Rosen C. Are duty hour regulations promoting a culture of dishonesty among resident physicians? Journal of Graduate Medical Education. 2013;5(4):553-555

[20] Comondore V, Wenner J, Ayas N. The impact of sleep deprivation in resident physicians on physician and patient safety: Is it time for a wake-up call? BMC Journal. 2008;50(10):560-564

[21] Philibert I. Sleep loss and performance in residents and nonphysicians: A metaanalytic examination.

Sleep. 2005;28(11):1392-1402

[22] Barger L, Ayas N, Cade CJ, Rosner B, Speizer F, Czeisler C. Impact of extended-duration shifts on medical errors, adverse events, and attentional failures. PLoS Medicine. 2006;3:e487

[23] Taffinder N, McManus I, Gul Y, Russell R, Darzi A. Effect of sleep deprivation on surgeons' dexterity on laparoscopy simulator. Lancet. 1998;352(9135):1191

[24] Dawson D, Reid K. Fatigue, alcohol and performance impairment. Nature. 1997;388(6639):235-235

[25] Landrigan C, Rothschild J, Cronin J, Kaushal R, Burdick E, Katz J, et al. Effect of reducing interns' work hours on serious medical errors in intensive care units. NEJM. 2004;351(18):1838-1848

[26] Temple J. Resident duty hours around the globe: Where are we now? BMC Medical Education. 2014;14(Suppl 1):S8
[27] Bolster L, Rourke L. The effect of restricting residents' duty hours on patient safety, resident well-being, and resident education: An updated systematic review. Journal of Graduate Medical Education. 2015;7(3):349-363

[28] Ahmed N, Devitt K, Keshet I, Spicer J, Imrie K, Feldman L, et al. A systematic review of the effects of resident duty hour restrictions in surgery: Impact on resident wellness, training, and patient outcomes. Annals of Surgery. 2014;259(6):1041-1053

[29] Bilimoria K, Chung J, Hedges L, Dahlke A, Love R, Cohen M, et al. National cluster-randomized trial of duty-hour flexibility in surgical training. NEJM. 2016;374(8):713-727

[30] Daskivich T, Jardine D, Tseng J, Correa R, Stagg B, Jacob K, et al. Promotion of wellness and mental health awareness among physicians in training: Perspective of a national, multispecialty panel of residents and fellows. Journal of Graduate Medical Education. 2015;7(1):143-147

[31] Voltmer E, Spahn C. Social Support and Physician's Health. Zeitschrift für Psychosomatische Medizin und Psychotherapie. 2009;55:51-69

[32] Dyrbye L, Shanafelt T, Balch C, Satele D, Freischlag J. Relationship between work-home conflicts and burnout among american surgeons: A comparison by sex. Archives of Surgery. 2011;146:211-217

[33] Whitley T, Allison E, Gallery M, Cockington R, Gaudry P, Heyworth J, et al. Work-related stress and depression among practicing emergency physicians: An international study. Annals of Emergency Medicine. 1994;23(5):1068-1071

[34] Balch C, Freischlag J, Shanafelt T. Stress and burnout among surgeons: 
Understanding and managing the syndrome and avoiding the adverse consequences. Archives of Surgery. 2009;144(4):371-376

[35] Shanafelt T, Oreskovich M, Dyrbye L, Satele D, Hanks J, Sloan J, et al. Avoiding burnout - The personal health habits and wellness practices of US surgeons. Annals of Surgery. 2012;255:625-633

[36] Zenasni F, Boujut E, Woerner A, Sulta S. Burnout and empathy in primary care - Three hypotheses. The British Journal of General Practice. 2012;62(600):346-347

[37] Wilkinson H, Whittington R, Perry $\mathrm{L}$, Eames C. Examining the relationship between burnout and empathy in healthcare professionals: A systemic review. Burnout Research. 2017;6:18-29 


\title{
Chapter 8
}

\section{Implementing Wellness Curriculum in Residency}

\author{
Nguyet-Cam V. Lam and Elspeth Black
}

\begin{abstract}
Transitions from medical school training to residency and then on to practice can be very challenging. An important but often overlooked aspect of medical education is the development of strategies to improve and sustain individual well-being so that trainees can successfully complete their training and transition into practice. In this chapter, we will be discussing ways in which physician and trainee well-being can be objectively assessed through the use of a burnout and work engagement index, as well as components of a wellness curriculum that can help to maintain and improve physician well-being across the continuum of training. Establishing a framework and wellness curriculum can help to prevent physician burnout and improve physician and trainee work engagement and well-being.
\end{abstract}

Keywords: resident physician well-being, wellness curriculum, wellness, residency, medicine, medical education, burnout, physician attrition, physician retention, quality improvement, medical school, education, sustainability

When health is absent,

Wisdom cannot reveal itself.

Art cannot manifest,

Strength cannot fight,

Wealth becomes useless, and intelligence cannot be applied.

—Herophilus, 300 B.C., Greek physician and pioneer of anatomy.

\section{Introduction}

Wellness is an essential training for new physicians, not only for their own well-being but for the well-being of their patients and the communities to which they belong. The reason why this should matter is obvious: depressed residents make 6.2 times as many medication errors per resident month as residents who are not depressed [1]. As mentioned in the previous chapter, physician wellness is also a public health issue because many US patients lose their physicians to suicide every year [2]. Burnout and depression in physicians, residents, and medical students is something we can objectively measure, study, and intervene to prevent. Residency programs are gradually adopting the idea of wellness into their culture and curriculum, with some initial research beginning to show the positive effects. Although 
the objective is the avoidance of burnout, the focus truly needs to be on a cultural shift away from rigorous study that requires self-sacrifice and an extreme work-life balance mismatch to a culture that is focused on education and the support of a balanced workplace that promotes wellness. Tenets of wellness need to be woven into the fabric of medical education, just as clearly emphasized as those of professionalism and patient safety.

As defined by Christina Maslach, burnout is a syndrome characterized by a loss of enthusiasm for work (emotional exhaustion), feelings of cynicism (depersonalization), and reduced personal accomplishment [3]. Although there has been much published on the subject of burnout in residency, there has been significantly less focused on the promotion of wellness [4]. Despite the fact that some programs have adopted wellness initiatives for their residents, many are still lacking a standardized, evidenced-based curriculum proven to improve outcomes. Similarly, some medical schools are beginning to integrate wellness practices into their curriculum, or at least into their extracurricular activities on offer. The issue of burnout must arise in medical school, as students entering medical schools are no different from their non-medical peers in terms of their mental health. Soon after starting their medical studies, the students' overall mental health state declines [5]. Studies have shown by making simple modifications in medical schools such as transitioning to a pass-fail model, strengthening student relationships with faculty and peers, and incorporating mindfulness and stress reduction techniques into training, we can prevent burnout and help manage preexisting depression [5].

One of the most intuitive places to start with a wellness curriculum is the encouragement and integration of physical activity into one's routine. There are numerous studies in the general population on the psychological, emotional, and mental well-being benefits derived from physical activity and time spent in an outdoors environment. Some studies have investigated the prevalence and possible incentivization of exercise for those in residency. One study that looked at internal medicine residents showed that although $35 \%$ of residents exercised daily prior to starting residency, only $4 \%$ were found to exercise daily during residency. About $41 \%$ of residents failed to meet the recommended guidelines for exercise as defined by the Department of Health and Human Services (HHS), and $80 \%$ reported a decrease in physical activity since starting medical training. Of greatest interest is that those residents who met the HHS guidelines for physical activity were less like to be burned out than their fellow residents (OR 0.38) [6]. A recent meta-analysis of trials investigating the impact of exercise on depression demonstrated a standardized mean difference of -0.62 (a moderate effect) in depression at the end of the study when comparing exercise with no treatment or a control intervention [7].

A study at the Mayo Clinic in 2013 investigated the specific effect of exercise on burnout in post-graduate training. They invited residents and fellows to participate in a voluntary, team-based 12-week exercise program. Prior to the intervention, only $31 \%$ of medical trainees were meeting HHS recommendations for physical activity. At the completion of the program, rates of adequate physical activity among the participants significantly increased (to 48\%) compared to nonparticipants (23\%). Most importantly, there was an increase in quality of life and a decrease in rates of burnout [8]. Another study identified that there are specific benefits to be gained by exercising outdoors, particularly in an alpine environment. The study showed that this environment improved symptoms of depression and quality of life measurements in those suffering from depression. Those with major depression were found to have lower levels of resiliency than their health counterparts at baseline, and that being physically active in an alpine environment was positively associated with an increase in resilience [9]. 
There has been significant research published to date on emotional intelligence (EI) and psychological resiliency as counterweights against workplace stressors and reactions to trauma in the workplace in nurses and other professions (not to mention the research on EI as a prognostic in various at-risk populations such as children with autism spectrum disorder). A study showed that there were individual positive curvilinear relationships between both EI and psychological resilience with post-traumatic growth in nursing students. Moderate-level EI and resilience were associated with the greatest growth $[10,11]$. This echoes similar studies in caregivers, where it was found that lack of resiliency was one of the main predictors of burden [12]. A separate study that investigated EI, resilience and burnout in pediatric and medicine-pediatric residents further supported the above conclusions. They found that self-compassion and mindfulness were positively associated with resilience and inversely associated with burnout. Interestingly, physician empathy and EI were not significantly correlated with burnout or resilience, suggesting that the impact of mindfulness and resiliency on burnout may be the reasonable focus to emphasize during curriculum development [13].

Failed attempts in the past have revolved around the individualized approach. The institution may ask what is wrong with the individual physician that causes him/her to become burned out. Rather than focusing on who is not succeeding, one should focus on what environmental factors are causing them to perform suboptimally. One factor that has previously been undervalued is that of leadership behaviors present in the environment. A recent Mayo Clinic study showed that every one-point increase on a 60-point leadership scale of a physician's immediate supervisor was associated with a $3.3 \%$ decrease in the likelihood of burnout and a $9.0 \%$ increase in satisfaction [14]. In addition, leaders can dramatically reduce the chance of burnout in their subordinate physicians by asking them what professional activity is most meaningful to them. Physicians who spend $20 \%$ of their time at work on something they value and find meaningful are at significantly lower risk for burnout [15]. Most residency programs are highly structured, both on a dayto-day level and overall, not leaving much time for flexibility or personal choice. Identifying opportunities to allow residents to spend some of their work hours doing the type of work that will continue to motivate and drive them through the work that is required by their programs is of immense importance.

Similarly, building a community at work is exceedingly important to overall physician well-being. Creating the space for interaction through literal spaces like physician lounges or through protected time during work hours for interest group meetings can be of significant value. Shared professional identity and support from one's peers have a demonstrated ability to reduce burnout [16]. An institution should be motivating its physicians to be efficient and provide good patient care, but sometimes these incentives can contribute to burnout and thus meet a self-defeating end. For example: the common payment structure of the base salary plus a productivity bonus. Productivity can be increased by seeing more patients per unit of time, ordering more tests, and by working longer hours. All three can contribute to reduced quality of care and increased physician burnout, despite being considered "productive" actions. The more recent shift in payment structure has been toward one of quality measures and patient satisfaction, but this model has yet to demonstrate actual improvement in quality and may not truly change the day-to-day experience of the overworked physician [17].

Regardless of the ultimate practices that they enter, we can significantly improve the outcomes if we integrate wellness into medical training as early as possible. There is a growing number of programs and health systems realizing this and creating wellness curricula for their residents. In the last few years, the American Medical Association (AMA) and the American Academic of Family Physicians (AAFP) have 
developed resources and even entire conferences devoted to the subject of physician wellness $[18,19]$. More often than a few years ago, one sees the term "resident wellness" on the websites promoting residency programs. Not only is the concept of physician wellness becoming more common, but so is the development of interventions to try to increase wellness. One such study at the Mayo Clinic demonstrated that a simple intervention of biweekly 1-hour discussion groups focused on mindfulness, shared experience, and small-group learning not only improved engagement at work and decreased burnout, but the effect was sustained 1 year later [20].

A meta-analysis in 2016 evaluated over 2000 articles regarding physician burnout (not residents, specifically), with 15 randomized trials and 37 cohort studies. The literature indicated that multiple techniques could be utilized that resulted in clinically meaningful reductions in burnout. When examined grossly, these studies showed the rates of overall burnout, emotional exhaustion, and depersonalization decreased following intervention by 10,14 , and $4 \%$, respectively [21].

The following year, a meta-analysis was completed specifically to investigate the efficacy of interventions in reducing resident physician burnout. Approximately $1 / 2$ of the studies included in the meta-analysis were specifically examining the effects of the 2003 \& 2011 Accreditation Council for Graduate Medical Education (ACGME) duty hour regulations. The collective results showed that reduced work hours were positively associated with a decrease in emotional exhaustion and depersonalization and no effect on personal accomplishment. The same were also decreased with other interventions including self-care workshops and a meditation intervention [22].

This collective research has been inspiring but less specific than desired to those in the medical education industry. Our aim was to implement a specific wellness curriculum, collect data illustrating its effectiveness, and present it to the readers in a comprehensive curriculum that is ready to implement. The data thus far collected has demonstrated the efficacy of our curriculum and we continue to strive to expand and add to the curriculum as the needs of our residents are established.

Our goal is to describe the most practical residency wellness education curriculum, to provide the tools for objective measurement of resident wellness and burnout, and to assist in career longevity of our young doctors. This should be a priority not only for those going through the process, but for patients and administrators, too. Physician burnout affects all groups and is a pervasive issue that affects healthcare at every level. Burnout leads to decreased quality of care, increased medical errors, decreased patient satisfaction, decreased productivity and time management, and a high rate of physician turnover [16]. It is in the best interests of patients, doctors, administrators and ultimately the larger community to devote time and resources to this issue.

\section{Assessment}

Whenever an intervention or curriculum is devised, it is equally important to properly develop an assessment and a timeline of monitoring to see if the desired effect or educational goals are being achieved. The following assessments were found to be reliable, validated, and appropriate to use to evaluate burnout and work satisfaction in the physician [3, 23, 24].

\subsection{Maslach Burnout Inventory}

The original Maslach Burnout Inventory (MBI) is a self-assessment tool composed of 22 items pertaining to occupational burnout. This tool assesses the three 
categories of burnout: emotional exhaustion, depersonalization, and personal accomplishment. In our suggested curriculum, the abbreviated Maslach Burnout Inventory ( $\mathrm{aMBI}$ ) is used, which adds an additional category of assessment that indicates satisfaction with medicine. This inventory can be periodically administered to monitor for increasing or decreasing levels of burnout in the individual or group.

Of the 12 items in the aMBI, they are separated as follows:

Emotional exhaustion is measured by three items. Higher scores are correlated with feeling more emotionally overextended and exhausted at work.

Depersonalization is measured by three items. Higher scores are correlated with a more frequent sensation of detachment and unfeelingness toward the care one provides at work.

Personal accomplishment is measured by three items. Lower scores are correlated with a reduced feeling of competence and success at work, and thus higher burnout.

Satisfaction in Medicine is measured by three items. Lower scores are correlated with a lower satisfaction in the career of medicine, and thus high burnout risk.

The MBI and the aMBI have been shown to have strong reliability, as well as being highly applicable and valid specifically in evaluation of family practice physicians $[3,23,25]$.

\subsection{Work engagement scale}

The Utrecht Work Engagement Scale (UWES) was developed to measure the positive opposite of burnout, which is work engagement [18]. Work engagement is defined "as a positive, fulfilled, work-related state of mind that is characterized by vigor, dedication, and absorption.” The UWES was originally developed with 17 items, however multiple studies have since streamlined and verified the validity of the UWES-9 [24, 26, 27]. The nine-item UWES measures vigor, dedication and absorption with three items each as follows:

Vigor: These three items aim to identify the subject's vigor. A higher score indicates that the subject has a high level of energy and mental resilience while working and that they are willing to invest effort in their work. They also demonstrate persistence in the face of difficulty.

Dedication: These three items aim to identify the subject's dedication. A higher score indicates that the subject has a greater sense of significance, enthusiasm, inspiration, pride, and challenge in their work.

Absorption: These three items aim to identify the subject's absorption. A higher score indicates that the subject is more deeply engrossed in their work. While at work, they experience time passing quickly and may have difficulty in detaching themselves from their work [24, 26-28].

UWES is an important metric to use in conjunction with the aMBI as work engagement has significant associations with productivity and mental health. Work engagement has been associated with numerous benefits including mental and psychosomatic health, intrinsic motivation, and positive attitudes toward work and the organization and high performance [28]. Furthermore, engagement is a more chronic, persistent state that is not associated with the day-to-day variations in work activity as may be other measurable indicators [24, 26-28].

\section{Curriculum}

Transitioning from medical school training to residency can be very challenging. An important but often overlooked aspect of graduate medical education 
is to help residents develop strategies to improve and sustain their well-being so that they can not only successfully complete their training but enter practice ready to overcome challenges. This section presents successful, practical initiatives from our family medicine residency program to assess, monitor, and enhance resident physician well-being. We will walk you through the steps of our wellness curriculum development and implementation. We will also present the spreading change from a single family medicine residency program to the hospital health network.

In August 2015, St. Luke's Family Medicine Residency Program in Bethlehem decided to study its resident burnout and wellness in preparation for a faculty development on physician burnout. Residents were assessed with the abbreviated Maslach Burnout Inventory (aMBI) [23, 29, 30]. The aMBI assesses the following three components: emotional exhaustion, depersonalization, and personal accomplishment. The "satisfaction with medicine" component was added to see how all these risk factors affect the residents' personal satisfaction with life in medicine.

Our analysis revealed that our residents' burnout levels approximated the national average, with emotional exhaustion being the predominant burnout category. Once we had obtained baseline data, the next step was to develop an intervention for ongoing wellness reinforcement and burnout prevention. At a program retreat in September 2015, we introduced the topic of physician burnout and wellness. We also discussed ways to construct resiliency to combat burnout. We encouraged residents to make goals to improve their well-being in several dimensions including physical, emotional, spiritual, and support goals. At this retreat, we organized a working group composed of faculty and residents to address wellness as a way to combat burnout. The working group was led by a faculty member passionate about wellness and one volunteer from each class (six residents per class). Following the creation of this group, a wellness curriculum was developed to include: monthly wellness education, engagement opportunities, and recognition of resident/faculty accomplishments. The main objective was to obtain a baseline wellness/burnout level in the intern year followed by reassessment every 6 months through the entirety of residency training. Using this data, we aimed to shape the culture and wellness initiatives to the needs of our program.

By the spring retreat in March 2016, we had made considerable progress in measuring wellness and burnout for our residents. Among other topics at the retreat, we presented "Practical wellness applications for our residency program to prevent burnout." Given that the highest category of burnout was emotional exhaustion, we specifically targeted the topics chosen for our residency retreat and wellness curriculum to focus on decreasing this particular risk. Although we focused on emotional exhaustion, our retreat still addressed the other objectives of decreasing depersonalization, enhancing a personal sense of achievement, and implementing healthy habits to combat burnout.

The wellness curriculum has two major components: hosting a residency retreat every 6 months and a monthly wellness meeting that was built into our previously established academic curriculum.

Common topics addressed at the biannual retreat are as follows:

- Communication and leadership skills

- Conflict management exercise

- Work-life balance and time management 
- Burnout and wellness in residency

- Meaningfulness at work

- The privilege of medicine

- Strategies for day-to-day challenges

- Teamwork and team building activities

- Giving and receiving feedback

Every month, a 1-hour time block is reserved during academic day to have a focused discussion either in small groups (five-six residents each) or with the entire group (all residents). Topics presented focus on enhancing wellness and decreasing burnout focus on three main categories: (1) importance of physician wellness, satisfaction in medicine, and healthy lifestyle, (2) addressing emotional exhaustion, depersonalization, and (3) encourage sense of personal achievement. Individual session's topics are summarized in Table 1.

In the small group format, especially in topics like wellness goal and goal setting, we use coaching technique to skill set in prioritization, self-care, and selfawareness [31].

Along with developing a curriculum to enhance wellness and decrease the risk of burnout, we also developed both a formal Physician Well-being Policy and a Resource Manual in March 2017 and May 2017, respectively. This was given to every intern at the beginning of their academic year to provide them the tools and resources they would need to optimize their wellness. In August 2017, we also added the Work Engagement Scale (UWES) to be used in conjunction with the aMBI [26].

Importance of physician wellness, satisfaction in medicine, and healthy lifestyle:

- Introduction to well-being to combat burnout

- Planning for life after residency in fellowship/practice/academia

- Nutrition and exercise

- Office exercise or walk in the park with your advisor

- Financial pearls

Addressing emotional exhaustion:

- Wellness goals and goal setting

- Share an inspirational quote

- Loving kindness guided meditation

- Work-life balance

- Time management

Addressing depersonalization

- Sharing a difficult patient encounter

- Mindfulness/reflection/empathy

Addressing low achievement

- Reflective writing on professionalism

- Cultural celebration

Table 1.

Wellness curriculum topics: 1 hour presentation and discussion. 


\section{Results}

Since August 2015, we have assessed the wellness/burnout of our residents every 6 months. Results were compiled looking at both our program as a group, as well as separated out by class. Of significant interest is that we were able to follow the class of 2018 from their intern year to graduation, thus collecting a full 3 years of data from the same cohort. Figures 1 and $\mathbf{2}$ and Tables $\mathbf{2}$ and $\mathbf{3}$ summarize the data for both the residency as a whole and for the class of 2018 from summer 2015 to winter 2018.

When examining the data from all residents as seen in Figure 1, we see decreasing indicators of burnout in three of four categories: emotional exhaustion decreased from 41 to $39 \%$, depersonalization decreased from 33 to $11 \%$, low satisfaction with medicine decreased from 22 to $19 \%$, and low achievement increased from 4 to $12 \%$. For the most part, there is a seasonal bimodal effect exhibited in most of these categories with increased burnout occurring in the winter with subsequent improvement in the summer months.

In Figure 2, the data from the class of 2018 was analyzed from the beginning of their intern year in 2015 until the winter before graduation in 2018. The risk factors of depersonalization, low achievement, and low satisfaction with medicine all decreased from $20-40$ to $0 \%$, while the emotional exhaustion category increased from 20 to

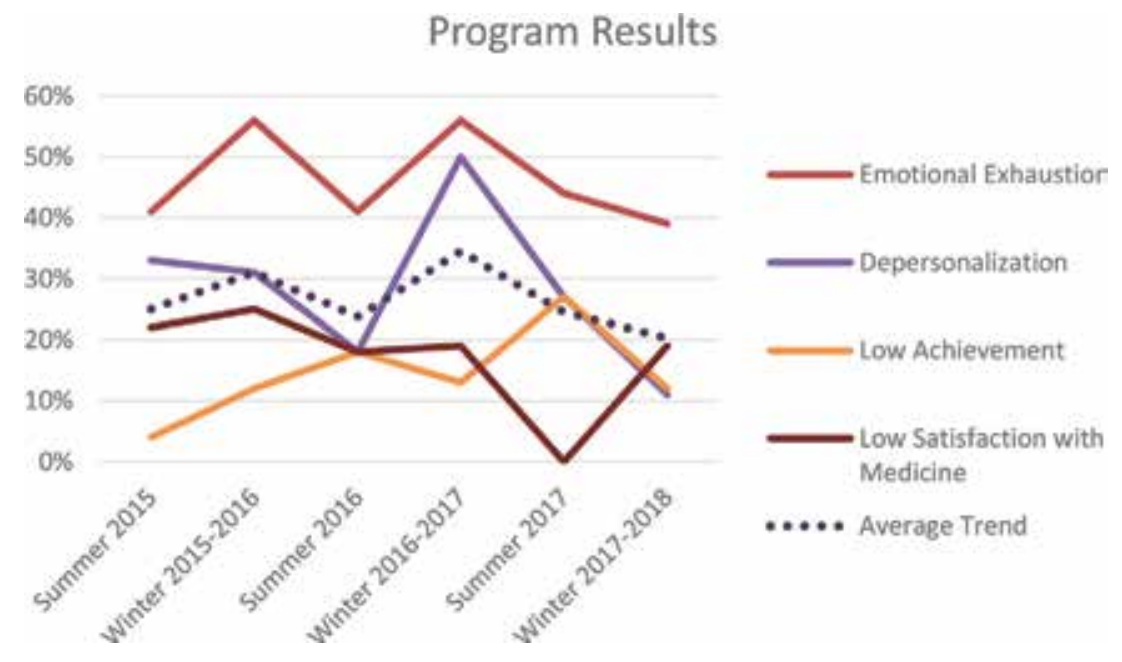

Figure 1.

Percent at risk per burnout category over time: program.

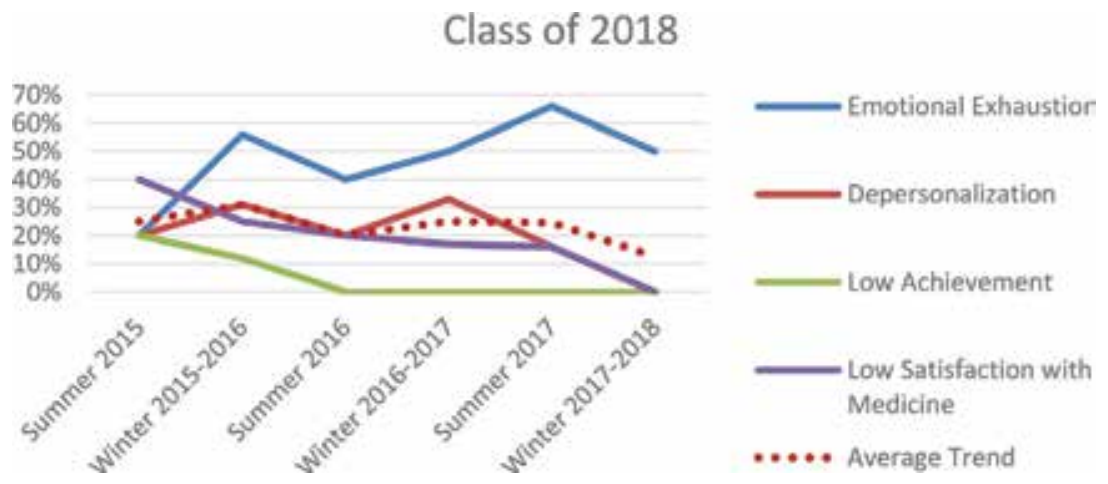

Figure 2.

Percent at risk per burnout category over time: class of 2018. 


\begin{tabular}{lcccc}
\hline Program data & $\begin{array}{c}\text { Emotional } \\
\text { exhaustion }\end{array}$ & Depersonalization & $\begin{array}{c}\text { Low } \\
\text { achievement }\end{array}$ & $\begin{array}{c}\text { Low satisfaction with } \\
\text { medicine }\end{array}$ \\
\hline Summer 2015 & $41 \%$ & $33 \%$ & $4 \%$ & $22 \%$ \\
\hline $\begin{array}{l}\text { Winter } \\
\text { 2015-2016 }\end{array}$ & $56 \%$ & $31 \%$ & $12 \%$ & $25 \%$ \\
\hline Summer 2016 & $41 \%$ & $18 \%$ & $18 \%$ & $18 \%$ \\
\hline $\begin{array}{l}\text { Winter } \\
\text { 2016-2017 }\end{array}$ & $56 \%$ & $50 \%$ & $13 \%$ & $19 \%$ \\
\hline Summer 2017 & $44 \%$ & $27 \%$ & $27 \%$ & $0 \%$ \\
\hline $\begin{array}{l}\text { Winter } \\
\text { 2017-2018 }\end{array}$ & $39 \%$ & $11 \%$ & $12 \%$ & $19 \%$ \\
\hline
\end{tabular}

Table 2.

St. Luke's family medicine residency program data.

\begin{tabular}{lcccc}
\hline Class of 2018 & $\begin{array}{c}\text { Emotional } \\
\text { exhaustion }\end{array}$ & Depersonalization & $\begin{array}{c}\text { Low } \\
\text { achievement }\end{array}$ & $\begin{array}{c}\text { Low satisfaction with } \\
\text { medicine }\end{array}$ \\
\hline Summer 2015 & $20 \%$ & $20 \%$ & $20 \%$ & $40 \%$ \\
\hline $\begin{array}{l}\text { Winter } \\
\text { 2015-2016 }\end{array}$ & $56 \%$ & $31 \%$ & $12 \%$ & $25 \%$ \\
\hline Summer 2016 & $40 \%$ & $20 \%$ & $0 \%$ & $20 \%$ \\
\hline $\begin{array}{l}\text { Winter } \\
\text { 2016-2017 }\end{array}$ & $50 \%$ & $33 \%$ & $0 \%$ & $17 \%$ \\
\hline Summer 2017 & $66 \%$ & $16 \%$ & $0 \%$ & $16 \%$ \\
\hline $\begin{array}{l}\text { Winter } \\
\text { 2017-2018 }\end{array}$ & $50 \%$ & $0 \%$ & $0 \%$ & $0 \%$ \\
\hline
\end{tabular}

Table 3.

St. Luke's family medicine residency class of 2018 data.

$50 \%$. Again, the seasonal bimodal pattern is noted in some risk factors, with peaks in winter and troughs in summer. Of note, during the residency training of this particular class significant stressors occurred including the departure of three residents during a 1-year time period as well as many interpersonal conflicts among residents.

The Work and Well-being survey was added as an additional study parameter in the summer of 2017 using the Utrecht Work Engagement Scale (UWES) [26]. So far, we only have two data points for summer 2017 and winter 2017-2018. The highest risk group which is not engaged at work and at risk for burnout has been constant at $33 \%$.

Our residency has been using validated tools to assess physician burnout and engagement by using the aMBI and UWES. Our wellness program is effective in addressing burnout and promoting wellness given the encouraging result in the residency data in decreasing physician burnout from 2015 to present time in 2018. This result helps to validate the effectiveness of our curriculum.

\section{Practicalities of implementing the resident physician wellness program}

The call for addressing wellness was stated in ACGME letter from August 2016 to the community: "While a commitment to resident well-being has been a shared 
focus for all of us engaged in graduate medical education, the topic of physician well-being has recently risen to the forefront within the broader medical community... Layered on top of these challenges is the stigma that many physicians in training and in practice encounter related to acknowledging that they need help, and to feeling supported and safe in asking for that help." [32] In March 2017, the ACGME revised the new section VI in Common Program Requirement asking each residency program to address physician well-being [33].

The implementation of a well-being program in residency does, however, come with challenges. It requires additional time and energy for the faculty wellness champion to rally support, train, organize, and execute the wellness program. Cost may also be an additional factor for the already tight residency budget.

In our residency program, we were fortunate to have the endorsement of the residency Program Director as well as the Designated Institution Official (DIO) in piloting wellness initiatives at our family medicine residency. We were able to get the support of faculty to promote wellness and participate in the curriculum for the residents. There is 1 hour per month set aside for the residents to participate in the wellness curriculum, and the involved faculty members spent about 1 hour per month on planning and preparing for the month's activities. Preparation for the biannual retreat (scheduled for a 5-hour block of time) usually begins 3 months prior to the retreat and requires an additional 1 hour per month toward preparations from the involved faculty. In the week leading up to the retreat, participating faculty may spend up to 3 more hours making specific preparations for organized discussions, team building games, other activities and food arrangements. Therefore, it takes about 40-50 hours per year for each faculty dedicated to wellness curriculum involvement.

In terms of cost, our residency utilized low-cost resources and implemented creative plans for our wellness activities. Wellness activities such as a walk in the park or exercising in the office can be accomplished without cost. The speakers and group leaders for the wellness topics were individuals from our own faculty or physician experts outsourced from the hospital network, again eliminating cost. At each monthly wellness meeting, our residency provided healthful snacks such as fruits, vegetables, healthy dips, crackers, and water which cost about $\$ 25-\$ 30$ per month. The retreat is usually held at a local park pavilion which costs approximately $\$ 50$ per retreat to rent. Food and materials necessary for each retreat costs approximately $\$ 300$. Therefore, in an academic year with two residency retreats, 12 wellness monthly meeting, the average annual cost to our residency budget is about $\$ 1000$ to support residency wellness.

Given the success of our residency program in addressing resident physician wellness, the DIO and GME has formed a physician well-being subcommittee to share the well-being initiatives we have developed, and to help spread the positive change to other residency programs within the network. The success in enhancing physician wellness goes beyond the work of individual residency program. It requires a multilevel approach from individual residents starting at orientation with assessment and personal goal setting to enhance well-being, to the program recognizing, assessing, organizing, and addressing well-being for residents, to the network endorsement by GME leadership and expanding wellness initiatives throughout the health network.

\section{Future directions}

Our residency program has seen many positive outcomes through promoting a culture of wellness and addressing physician burnout. Promoting physician wellness takes more than just a wellness curriculum. It requires efforts from individual 
physicians, residency faculty at the program level, and also support at institution level. On a national level, the ACGME and many other national organizations have called for awareness and continued support to promote physician well-being and decrease burnout risks. Ultimately, this will help to fulfill the quadruple aims of healthcare in enhancing patient experience, improving population health, reducing costs, and improving the work life of the health care providers.

As we continue to strive for a better future, we hope to build upon the excellent response to the wellness curriculum in our residency over the past 3 years. We will need to continue improving and modifying our approach to best meet the need of our residents. The structure of our current curriculum, including both monthly sessions and the content of the retreat is drawn directly from similar ideas present in the current literature [34]. As we continue to grow, we may consider the addition of required reading about wellness or introducing apps for mindfulness to the residents [35]. In term of topics, we could add in modules as suggested by Arnold et al. such as physician suicide, "I need help", or "dealing with difficult consultants and staff" [36].

Another area of focus for development would be addressing resiliency. A metaanalysis identified that the factors conferring the largest increase in resiliency were higher self-esteem, most positive attributional style, and lower perfectionism. Other factors with moderate impact on resilience include lower trait reappraisal and higher EI. This study thus identifies avenues for further development of curriculum modules in order to bolster well-being during residency through resiliency training [37].

In recent years, literature has focused on a discussion of work-life balance versus work-life integration [38-40]. It is important to realize that a well-balanced integration for professional and private life is an important goal for the new generation of doctor [41]. We can consider changing our curriculum topic of work-life balance to work-life integration to emphasize that the work life and personal life can mingle and integrate. The physician can find a sense of joy and enhance well-being when there are meaningful interconnections between the many existing roles in both work and personal life.

Among the many factors that could help improving physician well-being, coaching has been an effective tool in improving general well-being of employees and reducing burnout $[42,43]$. In our small group, we use coaching techniques to help residents with goal settings, self-awareness, and self-care. However, we can consider getting more formal training for faculty in coaching residents so that we can have similar training standards for our coaching techniques.

Given the above future directions, we will need to ensure that we continue to assess our physician wellness and evaluating all the initiatives to make sure that our curriculum is effective and validated. By continuing to measure burnout and wellness with the aMBI and the UWES every 6 months, we can monitor the wellness of our residents as well as assessing the effectiveness of our curriculum in improving well-being through time. There are many other validated instruments to assess work-related dimensions of well-being such as Oldenburg Inventory, Physician Work-life Study's Single-Item, Copenhagen Burnout Inventory, Stanford Professional Fulfillment Index, Well-Being Index, Patient Health Questionnaire-9 [44]. The choice of using any of these validated would be depending on the resource available for the residents or organization in term of cost and personnel to conduct the test and monitor the results.

\section{Conclusion}

Formal assessment of wellness and burnout and the development and maintenance of a wellness curriculum throughout medical education is essential to future 
physician well-being. Given that the major factors contributing to resident wellbeing are autonomy, competency, social connectedness and time away from work, we will need more than just the residency program to address these issues [45]. The promotion of resident wellness requires a multi-level approach involving the individual physician, the residency, and the systematic support at organizational and national levels to wholly address burnout and enhance well-being. The ACGME's Clinical Learning Environment Review (CLER) program requires residency to monitor physician well-being and burnout. It is recommended that the CLER can go further to expect institutions to have initiatives to enhance resident wellness and increase engagement through identifying suboptimal aspects of the learning environment and enhance resiliency in residency [46]. The health of our physicians is a far-reaching issue and must be addressed. Their longevity, productivity and happiness are heavily impacted by wellness and rates of burnout and can be greatly shaped throughout their medical education journey. The foundation has been built, but we must continue to fervently pursue the establishment of wellness as a pillar of medical education.

\section{Acknowledgements}

We would like to acknowledge the support of the St. Luke's University Health Network, with particular attention to those in the St. Luke's Family Medicine Residency Program and the Graduate Medical Education Department.

\section{Conflict of interest}

The authors have no conflicts of interest to disclose.

\section{Author details}

Nguyet-Cam V. Lam* and Elspeth Black

St. Luke's Family Medicine Residency Program, Bethlehem, PA, USA

*Address all correspondence to: nguyet-cam.lam@sluhn.org

IntechOpen

(C) 2018 The Author(s). Licensee IntechOpen. This chapter is distributed under the terms of the Creative Commons Attribution License (http://creativecommons.org/licenses/ by/3.0), which permits unrestricted use, distribution, and reproduction in any medium, provided the original work is properly cited. (cc) BY 


\section{References}

[1] Fahrenkopf A, Barger L, Lewin D, Edwards S, Landrigan C. Rates of medication errors among depressed and burnt out residents: Prospective cohort study. British Medical Journal. 2008;336:488. DOI: 10.1136/ bmj.39469.763218.BE

[2] Hampton T. Experts address risk of physician suicide. Journal of the American Medical Association. 2005;294(10):1189-1191. DOI: 10.1001/ jama.294.10.1189

[3] Maslach C, Jackson S, Leiter M, Schaufeli W, Schwab R. Maslach Burnout Inventory. Palo Alto, CA: Consulting Psychologists Press; 1986

[4] Eckleberry-Hunt J, Van Dyke A, Lick D, Tucciarone J. Changing the conversation from burnout to wellness: Physician well-being in residency training programs. Journal of Graduate Medical Education. 2009;1(2):225-230. DOI: 10.4300/JGME-D-09-00026.1

[5] Slavin SJ, Schindler DL, Chibnall JT. Medical student mental health 3.0: Improving student wellness through curricular changes. Academic Medicine. 2014;89(4):573-577. DOI: 10.1097/ ACM.0000000000000166

[6] Olson S et al. Burnout and physical activity in Minnesota internal medicine resident physicians. Journal of Graduate Medical Education. 2014;6(4):669-674. DOI: 10.4300/ JGME-D-13-00396

[7] Cooney G et al. Exercise for depression. Cochrane Systematic Reviews. 2013;19:1-157. DOI: 10.1002/14651858.CD004366.pub6

[8] Weight C et al. Physical activity, quality of life and burnout among physician trainees: The effect of a team-based, incentivized exercise program. Mayo Clinic Proceedings.
2013;88(12):1435-1442. DOI: 10.1016/j. mayocp.2013.09.010

[9] Ower C et al. The effect of physical activity in an alpine environment in quality of life in patients with psychosomatic disorders and health controls. European Archives of Psychiatry and Clinical Neuroscience. 25 July 2018. DOI: $10.1007 /$ s00406-018-0930-2

[10] Li Y, Cao F, Liu J. Nursing students' post-traumatic growth, emotional intelligence and psychological resilience. Journal of Psychiatric and Mental Health Nursing. 2015;22:326-332. DOI: 10.1111/jpm.12192

[11] McCrimmon A, Climie E, Huyn H. The relation between emotional intelligence and resilience in at-risk populations. Developmental Neurorehabilitation. 2018;21(5):326-355. DOI: $10.1080 / 17518423.2017 .1387873$

[12] Palacio C, Krikorian A, Limonero J. The influence of psychological factors on the burden of caregivers of patients with advanced cancer: Resiliency and caregiver burden. Palliative $\&$ Supportive Care. 2018;16(3):369-277. DOI: $10.1017 / S 147895157000268$

[13] Olson K, Kemper K, Mahan J. What factors promote resilience and protect against burnout in first-year pediatric and medicine-pediatric residents? Journal of Evidence-Based Complementary and Alternative Medicine. 2015;20(3):192-198. DOI: $10.1177 / 2156587214568894$

[14] Shanafelt TD, Gorringe G, Menaker $\mathrm{R}$, et al. Impact of organizational leadership on physician burnout and satisfaction. Mayo Clinic Proceedings. 2015;90(4):432-440

[15] Shanafelt TD, West CP, Sloan JA, et al. Career fit and burnout among 
academic faculty. Archives of Internal Medicine. 2009;169(10):990-995. DOI: 10.1001/archinternmed.2009/70

[16] Shanafelt TD, Noseworthy JH. Executive leadership and physician well-being: Nine organizational strategies to promote engagement and reduce burnout. Mayo Clinical Proceedings. 2017;92(1):129-146. DOI: 10/1016.j.mayocp.2016/10/004

[17] Shanafelt TD, Gradishar WJ, Kosty $\mathrm{M}$, et al. Burnout and career satisfaction among US oncologists. Journal of Clinical Oncology. 2014;32(7):678-686. DOI: $10.1200 / J C O .2013 .51 .8480$

[18] Physician Wellness: Preventing Resident and Fellow Burnout [Internet]. 2018. Available from: https://www. stepsforward.org/modules/physicianwellness [Accessed: Sep 6, 2018]

[19] Physician Health and Well-being Conference [Internet]. 2018. Available from: https://www.aafp.org/events/ fpwb-conf.html [Accessed: Sep 6, 2018]

[20] West C, Dyrbye L, et al. Intervention to promote physician well-being, job satisfaction, and professionalism: A randomized clinical trial. JAMA Internal Medicine. 2014;174(4):527-533. DOI: 10.1001/ jamainternmed.2013.14387

[21] West C, Dyrbye L, et al. Interventions to prevent and reduce physician burnout: A systematic review and meta-analysis. Lancet. 2016;388(10057):2272-2281. DOI: 10.1016/S0140-6736(16)31279-X

[22] Busireddy K, Miller J, et al. Efficacy of interventions to reduce resident burnout: A systematic review. Journal of Graduate Medical Education. 2017;9(3):294-301. DOI: 10.4300/ JGME-D-00372.1

[23] Rafferty et al. Validity of the Maslach Burnout Inventory for family practice physicians. Journal of Clinical Psychiatry. 1986;42:3. DOI: 10/1002/1097-4679(198605)42:3 <488::AID-JCLP2270420315.3.0.CO;2-S

[24] Schaufeli W, Salanova M, GonzalezRoma V, Bakker A. The measurement of engagement and burnout: A two sample confirmatory factor analytic approach. Journal of Happiness Studies. 2002;3(1):71-92. DOI: 10.1023/A:1015630930326

[25] Riley M, Mohr D, Waddimba A. The reliability and validity of the threeitem screening measures for burnout: Evidence from group-employed health care practitioners in upstate New York. Stress \& Health. 2016;34(1):187-193.

DOI: $10 / 1002 /$ smi.2762

[26] Schaufeli W, Bakker A, Salanova M. The measurement of work engagement with a short questionnaire. Educational and Psychological Measurement. 2006;66(4):701-716. DOI: 10.1177/ 0013164405282471

[27] Seppala P et al. The construct validity of the Utrecht Work Engagement Scale: Multisample and longitudinal evidence. Journal of Happiness Studies. 2009;10:459-481. DOI: 10.1007/s10902-008-9100-y

[28] Schaufeli W, Salanova M. Efficacy or inefficacy, that's the question: Burnout and work engagement, and their relationships with efficacy beliefs. Anxiety, Stress, and Coping. 2007;20(2):177-196. DOI: 10.1080/106158007021217878

[29] McManus IC, Jonvik H, Richards P, Paice E. Vocation and avocation: Leisure activities correlate with professional engagement, but not burnout, in a cross-sectional survey of UK doctors. BMC Medicine. 2011;9:100. DOI: 10.1186/1741-7015-9-100

[30] McManus I, Smithers E, Partridge P, Keeling A, Fleming P. A levels and 
intelligence as predictors of medical careers in UK doctors: 20-year prospective study. British Medical Journal. 2003;327:139-142. DOI: 10.1136/bmj.327.7407.139

[31] Schneider S, Kingsolver K, Rosdahl J. Physician coaching to enhance wellbeing: A qualitative analysis of a pilot intervention. Explore (New York, NY). 2014;10(6):372-379. DOI: 10.1016/j. explore.2014.08.007

[32] Nasca T. Letter to Members of the Graduate Medical Education Community. 2016. Available from: https://www.acgme. org/Portals/0/PDFs/Nasca-Community/ August2016NascaLettertoCommunity.pdf

\section{[33] ACGME Common Program}

Requirement [Internet]. Available from: https://www.acgme.org/Portals/0/ PFAssets/ProgramRequirements/ CPRs_2017-07-01.pdf [Accessed: Aug 21, 2018]

[34] Cornelius A, Cornelius B, Edens M. Increasing resident wellness through a novel retreat curriculum. Cureus. 2017;9(7):e1524. DOI: 10.7759/cureus.1524

[35] Runyan C, Savageau J, Potts S, Weinreb L. Impact of a family medicine resident wellness curriculum: A feasibility study. Medical Education Online. 2016;21(1):30648. DOI: 10.3402/meo.v21.30648

[36] Arnold J et al. An evidencebased, longitudinal curriculum for resident physician wellness: The 2017 resident wellness Concensus summit. The Western Journal of Emergency Medicine. 2018;19(2):337-341. DOI: 10.5811/westjem.2017.12.36244

[37] Johnson J et al. Resilience to motional distress in response to failure, error or mistakes: A systematic review. Clinical Psychology Review. 2017;52:19-42. DOI: 10.1016/j.cpr. 2016.11.007
[38] Schwingshackl A. The fallacy of chasing after work-life balance. Frontiers in Pediatrics. 2014;2(26):1-3. DOI: $10.3389 /$ fped.2014.00026

[39] Vliagoftis H. Work-life balance: How can we achieve it within the work environment? Frontiers in Pediatrics. 2016;4(40):1-3. DOI: $10.3389 /$ fped.2016.00040

[40] Williams JC, Berdahl JL, Vandello JA. Beyond work-life "integration". Annual Review of Psychology. 2016;67:515-539. DOI: 10.146/annurev-psych-122414033710

[41] Buddeberg-Fisher B, Stamm M, Budddeberg C, Klaghofer R. The new generation of family physicianscareer motivation, life goals and work life balance. Swiss Medical Weekly. 2008;138(21-22):305-312

[42] Duijts SF, Kant I, van den Brandt PA, Swaen GM. Effectiveness of a preventive coaching intervention for employees at risk for sickness absence due to psychosocial health complaints: Results of a randomized controlled trial. Journal of Occupational and Environmental Medicine. 2008;50(7):765-776. DOI: 10.1097/ JOM.0b013e3181651584

[43] Gazelle G, Liebschutz JM, Riess H. Physician burnout: Coaching a way out. Journal of General Internal Medicine. 2015;30 (4):508-513. DOI: $10.1007 /$ s11606-014-3144-y

[44] National Academy of Medicine. Validated Instruments to Assess WorkRelated Dimensions of Well-Being. 2018. Available from: https://nam.edu/ valid-reliable-survey-instrumentsmeasure-burnout-well-work-relateddimensions/ [Accessed: Sep 14, 2018]

[45] Raj K. Well-being in residency: A systematic review. Journal of Graduate Medical Education. 2016;8(5):674-684. DOI: 10.4300/JGME-D-15-00764.1 
[46] Jennings M, Slavin S. Resident wellness matters: Optimizing resident education and wellness through the learning environment. Academic Medicine. 2015;90:1246-1250. DOI: 10.1097/ACM.0000000000000842 


\title{
The Importance of Post-Doctoral Program to GME in an Academic Medical Center
}

\author{
Tracy L. Butryn, Parampreet Kaur, Vikas Yellapu, \\ Alyssa Green and James Dalkiewicz
}

\begin{abstract}
Continuous advancements in the medical field and the need to promote scientific evidence has increased the concern for educators to ensure that physician trainees are provided with the appropriate tools and experiences to develop the skills necessary to enhance scientific discovery. To address this requirement, the Accreditation Council for Graduate Medical Education (ACGME) implemented core competencies, inclusive of scholarly activity requirements, for accreditation of Graduate Medical Education (GME) programs. These changes have challenged institutions to educate differently and incorporate scholarly activity and research into their curriculum through novel and creative approaches. One such mechanism is the development of post-doctoral research programs which utilize research fellows to provide the necessary support for research productivity across multiple specialties. In the following chapter, the authors will provide some background information on the goals and function of the ACGME, detail the development of the new research requirements, the utilization of post-doctoral research fellows to support the scholarly activity requirement laid out by the ACGME, and potential measures of performance and success.
\end{abstract}

Keywords: GME, post-doctoral, fellow, research

\section{Introduction}

Advancements in field of medicine have long since been established through scientific inquiry. Some would argue that the practice of medicine should be predominantly evidence based [1-3]. As the gap between medical practice and scientific evidence continues to broaden, it became necessary to ensure that the practice of medicine fits hand in hand with scientific discovery as well as staying abreast of current standards of care based on scientific evidence. The question then becomes at what point in medical training should this integration be made-during medical school, residency, or as an attending?

Over a decade ago, the Accreditation Council for Graduate Medical Education (ACGME) began to address the issues surrounding the core competencies necessary to ensure a well-rounded, competent physician by making compliance with these requirements as a condition of accreditation for Graduate Medical Education (GME) programs [4]. These core competencies include: patient care, medical 
knowledge, practice-based learning and improvement, professionalism, interpersonal skills and communication, and systems-based practice [4]. In line with these competencies, scholarly activity serves to integrate research and scientific discovery into the educational development of resident physicians [5]. These new requirements are being enforced as more studies are starting to show that participating in scholarly activity increases the scope of evidence based medicine and increases the quality of patient care among all health care professionals [6-9].

With the institution of the scholarly activity requirement comes the challenge of implementing this into the resident curriculum [10]. One novel approach is through the establishment of a post-doctoral research program. In the following chapter, the authors will provide some background information on the goals and function of the ACGME, detail the development of the new research requirements, and give the reader a look into how our institution has developed a program to ensure to support the scholarly activity requirement laid out by the ACGME. It will conclude with ideas on measuring the success of the program.

\section{The ACGME and Research}

Medicine is both an art and a science. This requires the ability to think critically, evaluate the literature, appropriately assimilate new knowledge, and practice lifelong learning. Physicians convey a distinct clinically focused perspective and perform a very important role in the advancement of evidence-based practice through medical research. They present issues vital to the enhancement of quality in patient care, but in the past have not been properly trained to utilize their unique perspective to enhance the field of medicine through research. GME programs and faculty must create an environment that fosters the acquisition of such skills through training programs and scholarly activity focus.

The ACGME and the American Board of Medical Specialties devised six core competencies for all physicians to address community expectations for advancements in science and thus medical care. This was indicative of the recognition by such agencies that there was a critical need for change in the way we educate and prepare our future physicians. These new requirements are being enforced as more studies are starting to show that participating in scholarly activity increases the scope of evidence based medicine, and increases the quality of patient care among all health care professionals $[6,7]$. A recent meta-analysis has shown that medical students who participate in research are three times more likely to show interest in research, six times more likely to engage in research, and twice as likely to outperform academically [6]. Additionally, involvement of physicians during and after medical school education have shown promising growth in research activities and publications, $[4,6]$ thus it is clear that fresh strategies are needed to grow the size and diversity of the physician-scientist pipeline [7]. Overall, there has been a change in focus to concentrate on the outcomes of such education in a measurable way, as well as collaboration across specialties using an institutional versus programmatic approach to graduate medical learning [4].

\section{ACGME accreditation}

As the educational models have shifted, scholarly activity in the form of research and quality improvement has become increasingly important in the eyes of the ACGME yet incorporating such activity onto Residency programs remains a challenge. In fact, issues surrounding inadequate scholarly activity are a common 
citation for many programs, especially Family Medicine residencies [11]. Across the United States, medical residency and fellowship programs are challenged to remain in compliance with scholarly activity requirements set by the ACGME. The ACGME uses Web Accreditation Data System (WebADS) to track and update faculty scholarly activity [12]. Additionally, since 2014 there was a transition to the next accreditation system (NAS) to promote evidence-based activity and improve reporting. These changes are indicative of the way the ACGME assess Graduate Medical Education (GME) programs [12]. Since the implementation of NAS, all ACGME programs have been required to submit evidence of scholarly activity of both residents and faculty on an annual basis, allowing the Residency Review Committee (RRC) to track performance and identify inadequacies with real-time intervention [12]. The increased frequency of reporting scholarly activity has created new challenges within GME programs. More than ever there is a need for synergetic and integrative approach between the Program Director, Chair, Designated Institutional Official, Faculty, and coordinator to address these challenges [12].

In response to these changes and the importance of receiving and/or maintaining accreditation, several Residency programs have had to modify their educational models and methods of measuring performance and outcomes [4]. To ensure accreditation standards are consistently met, there must be a spotlight on adequate performance measures, thus institutions need to ensure accountability and quality

ACGME requirements pertinent to research

- Knowledge of the basic principles of research

- How to evaluate research

- How research is applied to patient care

- Publication or presentation of scholarly activity.

- Development of leadership skills

- Strengthening interpersonal skills

- Strengthening communication skills

Table 1.

List of requirements that residents should to meet prior to graduation per ACGME.

\begin{tabular}{|c|}
\hline - Asking the right research question \\
\hline $\begin{array}{l}\text { - Writing Protocol } \\
\text { - IRB Submission }\end{array}$ \\
\hline $\begin{array}{l}\text { - Collaboration with non-clinical departments to obtain data } \\
\text { - Collaboration with students/residents to chart review }\end{array}$ \\
\hline $\begin{array}{l}\text { - Using proper statistical methods } \\
\text { - Understanding results }\end{array}$ \\
\hline - Dedicated time \\
\hline
\end{tabular}

Figure 1.

Barriers faced by residents when performing research. 


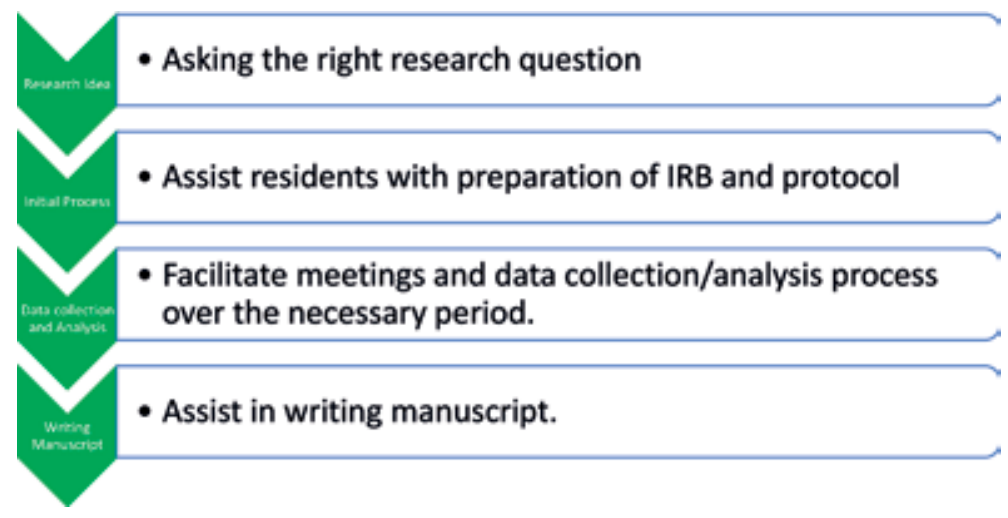

Figure 2.

Process with a post-doctoral fellow.

improvement are at the forefront of their culture [6]. Practically speaking, GME programs must have a clear understanding of what the scholarly activity requirements are with mechanisms for assessing performance, the ability to collect and report a full accounting of scholarly activities to the ACGME, and program leaders must stay abreast of new and upcoming changes in scholarly activity requirements to ensure compliance [12]. Scholarly activity metrics have historically included number of grant submissions, grants awarded, publications submitted, presentations, and awards which were tracked before and after the implementation of a new program which was then titled the "Baby Steps" program for faculty and residents [6]. The Resident Review Committee (RRC), in multiple specialties, has provided guidelines on how it interprets scholarly activity for compliance with the Program Requirements (Table 1). These guidelines include definitions and expectations, as well as examples of scholarly activities recognized by the Review Committee [11] (Figures 1 and 2).

\section{Post-doctoral program in GME}

Given these new competency models, the increasing need to adequately measure performance/scholarly activity and the growing value of being an accredited program, adaptation in the professional development of our future physicians is critical. The ACGME requires all accredited residency training programs to facilitate resident scholarly activities, thus GME programs must remain agile in their approach to meet these demands through novel educational approaches as well as flexible infrastructures $[13,14]$. This can be done by having dedicated research blocks or protected time from clinical responsibilities to complete research projects; however, clinical education is still the focus of Graduate Medical Education in most training programs. Residency programs are challenged with designing the structure, process, and outcome evaluation of the ACGME research requirement $[8,15]$. There must be support provided by the institution to ensure protected time for residents and faculty to focus on scholarly activity production $[8,14,16,17]$ Figure 1.

One mechanism for driving such educational models and support mechanisms is the use of a post-doctoral research training programs. Post-doctoral research fellows can add value to residency and fellowship programs at teaching hospitals by providing support in research productivity across multiple specialties $[18,19]$ Figure 2. Productivity generated by post-doctoral research fellows align well with what the ACGME defines as scholarly activity. According to a 2012 study published in the Journal of Graduate Medical Education (JGME), the addition of a mentored 
post-doctoral researcher was associated with improvements to both resident and faculty research activities [20]. While the original intent of the post-doctoral role was prominently to benefit the trainee through active mentorship, and to help secure future career goals through acquisition of additional skills and credentials, these roles have become essential in most research settings [18, 21] such as Graduate Medical Education in which scholarly activity is a core component. Therefore, it is not surprising that such post-doctoral research programs have become a key element within many institutions serving as a distinct phase of career succession in most scientific fields [21]. Post-doctoral training has been considered a critical component of research training, allowing the acquisition of the necessary skills to become an independent researcher [19]. A post-doctoral program enriches any robust research enterprise at an academic center. Overall, post-doctoral fellows play a crucial role in an academic institute; they supplement the research expertise of the faculty by teaching and advising undergraduates, residents, and fellows; contribute new research and quality improvement techniques; collaborate with other institutions; and help manage the daily research operations. They have deep expertise and knowledge to support faculty and residents, while also enhancing their own professional skills during their training. This type of environment boosts their research knowledge and skills for career advancement, developing a pipeline of future research-minded clinicians.

The post-doctoral scholars should be able to demonstrate broad knowledge of the research area and should be able to understand the gaps, limitations and challenges within the research [22]. Post-doctoral programs are common and have served as a standard stepping stone in other doctoral pathways. In other professions a post-doctoral position is used as a bridge to develop professional gaps that were not taught in an academic setting. ACGME has always encouraged the development of programs that will allow residents to develop core competencies. It recognizes the diversity of postdoctoral education programs, and anticipates that programs prepare specialists for a variety of roles, including contributors to clinical care, scientists, and educators. It is expected that the program's scholarship will reflect its mission(s) and aims, and the needs of the community it serves. For example, some programs may concentrate their scholarly activity on quality improvement, population health, and/or teaching, while other programs might choose to utilize more classic forms of biomedical research as the focus for scholarship [22]. There have been institutions that have developed various strategies to tackle this. A Mayo Clinic family medicine residency program in Arizona had implanted a "collaborative care" curriculum that allowed for critical thinking, cost-benefit analysis, professional development, mentorship, and leadership development [23]. This program has been considered a success and many programs have adapted this curriculum. However, this curriculum only targets family medicine residents in their final year. These core competencies should extend over the course of the residency program. Our program is set up to combine all the aspects of the "collaborative care" curriculum and have it available to residents and fellows at all times.

At our institution, the post-doctoral research program was created to help health science graduates gain research and leadership experience in a clinical and nonclinical setting, while also assisting residency programs meet their GME requirements. Few of the skills that GME requires from residents include: working in groups, understanding risk benefit analysis; engaging in quality improvement projects; developing strong leadership skills, and participation is scholarly activity. We will discuss in greater detail how the formation of a post-doctoral research program employing recent medical graduates can assist the GME in achieving their requirements as well as cultivating competitive residency candidates armed with the skills, knowledge and professional competence to continue contributing to future scholarly activities. We will also address potential metrics to gauge success of the program. 
The post-doctoral research program is a two-year program dedicated to providing hands-on experience in clinical and quality research. Our program creates a research group that enables them to practice working in a professional group outside a clinical setting and promotes leadership growth. With the introduction of a postdoctoral candidate, we were able to create a liaison between residents and various key research departments. This allowed for residents the opportunity to work with a diverse group of healthcare professionals in a research setting. There have been studies that show that residents that engage in scholarly activity find their residency more fulfilling and are happier [24]. In one of the surveys done in Department of Radiology and Radiological Sciences at the Medical University of South Carolina, majority of faculty members (86\%) and residents (51\%) thought that residents should be required to engage in a scholarly activity [14]. Many studies expressed a key component was the availability of mentors who are knowledgeable in research $[10,14,25]$. Our program has created this setting with program directors and other key research personnel as part of a council that assist in scholarly activity throughout the network. By creating opportunities for residents to interact with different members at different levels within the network allows them to learn about system organization and culture outside of a clinical environment [26]. It is important that residents understand the challenges and see how these challenges are overcome outside of a clinical situation. Interacting with other health professionals and administrators will instill a sense of teamwork that extends beyond the patient room, an effective trait of a strong leader. Residents are given a chance to develop other skills during residency, and through these valuable experiences can be well rounded physician leaders in the future. Studies have shown that there is a significant relationship between strong physician leaders and improved outcomes among patients [11].

The more obvious benefit of the post-doctoral research training program is that individuals in the program can be trained and placed in various departments where there is a need for assistance with keeping up with accreditation requirements. Our program trains post-doctoral candidates in the areas of IRB preparation, project feasibility, research statistics, and research presentations. They help design the research protocol early from the inception of idea and team formation, estimate sample size, safely conduct the research, analyze the data, and eventually write the manuscript. Along with this, they assist in IRB review, search and evaluate the literature, and present at various scientific conferences. None of these skills are taught in medical school, yet they play a vital role in the clinical setting. The leadership and management skills and techniques honed during their training period also facilitate effective team work and establish collaborations among various specialties/industry to conduct multidisciplinary research. Creating exposure to these skills and resources early in a physician's career can create a well-rounded clinician with strong leadership skills. By establishing a program with fellows that are knowledgeable in these areas it benefits everyone within the network. Assistance from post-doctoral research fellows provides a concrete structure and resource for faculty, residents and fellows; it allows them to generate further research and quality improvement projects; and it generates a significant impact on GME requirements for residency. Their research support and mentorship can play a significant role to maintain an active research programs in the teaching institutions. They are considered the major engine of driving the research in an academic center [27].

Post-doctoral program can give an exponential growth in the publication pool in an academic center. The cost of capitalizing in this kind of programs may be initially intimidating for institutions, yet the productivity and growth in research centers who adopt such a model is extremely valuable. Without active backing from faculty and post-doctoral scholars, advances in patient care, our departments, our institutions and society can experience significant inaction. The fellows themselves will meet the 
ACGME core competencies, the residents that take advantage and interact with the program will develop key skills, and the network will benefit from the increase in scholarly activity. In this instance, the fellow could act as a research coordinator of sorts and help educate faculty on the importance of their involvement in the process as well as facilitating collaboration among clinical staff and resident/fellows. The research fellow can also provide guidance on appropriate study design, statistical methods, as well as manuscript writing. Even in the case of residency programs that have no issue meeting GME requirements for scholarly activity, the post doc fellow can provide support throughout the research process as a coordinator, from IRB approval to manuscript writing, thus warranting clinicians with their clinical duties and alleviating some of the unknown and stress associated with the process of conducting research.

Many medical professionals matriculate without engaging in any scholarly activity so as a resident it may be the first time they are required to participate in research [28] which without proper mentorship can be daunting task especially with the adjustment to clinical responsibilities. One potential way to counter this is to involve residents early in the research process starting with quality improvement projects. The importance of patient safety and quality health care cannot be overstated. While the resident is becoming accustomed to their new roles and responsibilities as an integral part of the health care team, it is reasonable to also stimulate their curiosity in research and innovation. As residents are often the "eyes and ears" of attending physicians, they have a unique perspective on quality and patient safety issues. Utilizing their perspective and linking them with good mentors can accomplish the objective of all involved.

ACGME outlines that programs must engage residents in projects the challenge them and enhance their skills outside of clinical practices. Residents are expected to participate in identifying system errors and implementing solutions. Working outside of patient care in research and QI allows them to broaden their scope to other systems at play in the health network and possible implement efficient solutions. Our ongoing QI/research projects provide an excellent QI activity and even awards exceptional QI projects every year. Residents get first-hand knowledge regarding the principles of high value care, cost efficiency and healthcare utility by participating in these QI projects. Many QI projects also require residents to have a strong grasp of testing modalities, first line management, and diagnostic testing metrics (specificity, sensitivity, negative predictive value, etc.). While these topics are briefly discussed in board exams and in medical schools, still participating in QI projects allows them to develop skills needed to critically assess these topics. It is vital that these skills are acquired prior to graduating from residency. This importance stems from the new measures and metrics that hospitals and insurance companies now look at for every physician. Ever since the implementation of Medicare there has been a premium placed on high value that is beneficial for both patients and hospitals. This has created a challenging position for practicing physicians. Physicians are constantly being forced to make cost effective decisions, even though their training and beliefs have guided them to make decisions that are beneficial for patients at any cost. Therefore, it is vital that residents are exposed to this expectation prior to graduation so they can navigate better when seeing patients as an attending physician.

Along with the post-doctoral research program, we have created a collaborative environment that is highly encouraging for such scholarly activities. The postdoctoral research fellow program has developed a solid framework and resources for residents to use and build upon. A survey study from a family medicine residency in Wisconsin showed while $85 \%$ of residents believed research was important only $8 \%$ were active in research. One of the biggest reasons for this discrepancy was the lack of time and resources available to the residents [25]. Having a research fellow to assist with various research tasks can help alleviate the pressure and 
time constraints on residents. Innovation is of increasing importance in Graduate Medical Education which includes new ways of increasing resident awareness and participation in research $[29,30]$. Our program is an innovative approach in increasing scholarly activity and interest among residents. Residents that can participate in these scholarly and leadership activities tend not only to be more satisfied with their career but also more confident in their knowledge base. Another key ACGME criterion for residents to develop prior to graduation includes professional development. It has been shown the professionalism goes hand in hand with high standard of clinical care [31]. The post-doctoral research program creates an excellent opportunity for the fellows and residents interacting with the fellows to develop professional standards and relationships.

With the implementation of a program such as this, there comes a necessity to objectively evaluate its utility as the effectiveness of such programs remains a topic of debate due to a need for consistent and effective methods of measuring performance. That said, it has been reported that there are over 50,000 post-docs in the United States alone regardless of the inconsistency in opinion as to whether such training programs are beneficial [21]. The importance of creating more description and structure to the training environment has been acknowledged by various professional organizations including National Postdoctoral Association and NIH. According to a recent publication, a project was started to develop a list of competencies without any comparison to any previous competencies. The 10 core competencies for assessment of post doctorates were established regardless of discipline or career route; broad conceptual knowledge of a scientific discipline, deep knowledge of a specific field, critical thinking skills, experimental skills, computational skills, collaboration and team science skills, responsible conduct of research and ethics, communication skills, leadership and management skills, and finally, survival skills. Further, each competency was multidimensional and could be divided into sub competencies for the rubric [32]. These core competencies not only contribute to their professional research development but can also prepare them for leadership roles within the organization and pay dividends in complementing the individual's scientific research program.

Based on this evidence, a focus on the establishment of standards and expectations of the trainee, as well as the mentor, is a critical and necessary piece of these post-doctoral training programs and their continued pervasiveness [21]. There is literature to suggest development of some sort of research curriculum with faculty mentorship and regular research meetings as a method to not only ensure that residents meet their scholarly activity requirements [33], but it also aids in tracking the progress and completion of these projects [34]. The post-doctoral fellow can be an integral part of this program which can accomplish the goal of facilitating mentorship relationships, tracking and completing scholarly activity.

Another option used by the department of Anesthesia at Pittsburg Medical Center is implementing a scoring system to objectively weight the difficulty, significance, and level of resident involvement in scholarly activity [35]. They presented a novel way to measure their scholarly activity, which could be utilized by our program to determine whether the post-doctoral research program is accomplishing the goal of increasing scholarly activity in their respective departments. It also allows for objective measurement of the degree of post-doc, resident and faculty involvement. This data could be used to determine where additional resources and support can be distributed to ensure everyone is doing their part. This scoring system can also give data on the quality, complexity and significance of the work being produced.

In 2000, the U.S. National Academies' Committee on Science, Engineering, and Public Policy (COSEPUP) reported and concluded that there is far too much variability in post-doctoral training programs, and recommended the following: (1) development of well-defined goals, policies and standards; (2) institutional recognition, 
status, and compensation to support such programs; and (3) occupational counseling to prepare post-docs entering the workforce [21]. If the aim of the ACGME is to clearly define expectations for scholarly work, coupled with how to adequately document these initiatives [36], it seems inherent to align not only the residency programs with these goals, but also the support systems such as post-doctoral training programs with clearly defined goals and expectations, and adequate scales of measurement. In a sense, the fundamentals housed within a residency program should not significantly differ from those within a post-doctoral research program as it pertains to scholarly activity. Besides these competencies, it has been shown in various studies that when a post graduate medical student participates and authors in various research projects, they tend to show higher match rates in their residency spot [37].

A basic conceptual framework of post-doctoral research programs should include institutional support both financially and culturally, adequate mentorship, clearly defined goals and expectations with corresponding measures of performance, opportunities for utilization of critical thinking skills; forums for education and didactics with timely review and feedback on current and future projects; and overall leadership and professionalism development. These programmatic characteristics are very much aligned and/or mirror images of the programmatic needs for GME as evidenced in the Clinical Scholars Program that was implemented at the Trident/Medical University of South Carolina (MUSC) Family Medicine residency over two decades ago [34]. Aligning such programs allows for shared responsibility for meeting scholarly activity requirements and allows residents and attending physicians to pull support from the post-doctoral trainees while focusing on clinical responsibilities, with increased scholarly activity output as the outcome. Yet the question remains, what is the best measure of such academic performance, and the overall business case for support staff such as post-doctoral research trainees.

It has been a longstanding tradition to measure scholarly activity performance and overall academic performance via publication lists and scholarly activity point systems. It has been shown that the best predictor of sustainable future scientific success was the number of publications completed as a post-doctoral trainee [19]. This is also used in the hiring process for new faculty in most Institutions, with scientific advancement being at the forefront of the institutional mission, thus assessing candidates by how likely they will advance the field of medicine [38]. With this in mind, it seems inherent that the incorporation of post-doctoral research fellows to support such scholarly activity output is beneficial. That said, it is critical that academic success is not simply measured by objective numbers, but also the quality of such publication output, as well as other diversified metrics beyond raw numbers of publications, citations, and impact factors which are outdated measures dating back to the mid-twentieth century [38]. Several institutions have developed their own creative measurement tools, aligning clinical goals with research and educational activity that are continuously reviewed to drive strategy and early identification of any issues to be addressed, understanding that academic success cannot be evaluated by strict numbers [38]. This new-age form of measuring academic achievement requires a wide-range team of faculty, program directors, administration and leadership, mentors, and trainees comprised of residents, fellows, and Post-Docs with each component being an active contributor to scholarly activity output. Therefore, defining the expected number of publications and the level of contribution of the researcher, coupled with providing adequate infrastructure and support, as well as regular review of progress toward meeting these goals, are all necessary components of a post-doctoral research program [19] and the overall goal of developing future physician researchers.

The development of future physician-investigators is especially important as it has been noted that there is a decline in the ability to recruit and retain clinical researchers who are adequately trained and qualified [39]. The post-doctoral 
research training pathway is even more critical given this notion, as such academically focused instructional programs are essential to developing a pipeline of trained physician researchers. Academic medicine is necessary to continuously test and reform clinical practice and develop new treatment options, and its future is dependent on preparing our trainees and junior faculty for such academic missions [7]. Obstacles in career advancement within the academic setting stem primarily from inadequate training and preparation, inadequate support for research, and clinical performance pressures [7]. Incorporation of a post-doctoral research program fosters growth academically by alleviating these barriers as such programs not only provide additional training and education within the field of research, but also provide an additional layer of research infrastructure support to existing faculty and GME programs thus allowing these existing clinicians to focus on clinical responsibilities. That is not to say that the faculty is not to be intimately involved in such post-doctoral research training, as their mentorship and support of scholarly activity are critical to the academic mission. For example, young trainees need faculty mentors to offer career guidance and support, as well as protection from moving (and oftentimes competing) institutional priorities [7], always ensuring research and scholarly activity are a primary component of overall goals to ensure future academic success.

Although, there has been an acknowledgement of the significance of clinical scientists in evidence based medical practice, still there has been a decline in interest to pursue lengthy medical and research training among medical graduates. The postdoctoral research fellowship provides the baby steps toward being an independent investigator, which can eventually demonstrate a unique role in medicine by connecting the space between scientific research and clinical medicine in their future careers. It can also reduce the training time to develop a successful physician scientist as compared to $\mathrm{MD} / \mathrm{PhD}$ programs while maintaining the objectivity of training.

\section{Conclusion}

In conclusion, the authors have presented a novel way to ensure the attainment of scholarly activity requirements for GME programs, as well as a unique way to stimulate academic and scientific discovery among faculty and residents. There was presented some literature on innovative ways to the post-doctoral research program can increase scholarly activity and support the efforts of the GME. We hope that our experience can be duplicated at other institutions.

\section{Author details}

Tracy L. Butryn*, Parampreet Kaur, Vikas Yellapu, Alyssa Green

and James Dalkiewicz

St. Luke's University Health Network, Bethlehem, United States of America

*Address all correspondence to: tracy.butryn@sluhn.org

\section{IntechOpen}

(C) 2019 The Author(s). Licensee IntechOpen. This chapter is distributed under the terms of the Creative Commons Attribution License (http://creativecommons.org/licenses/ by/3.0), which permits unrestricted use, distribution, and reproduction in any medium, provided the original work is properly cited. (cc) BY 


\section{References}

[1] Sackett DL. Evidence-based medicine. In: Seminars in Perinatology. Philadelphia, PA: Saunders; 1997;21:1

[2] Rosenberg W, Donald A. Evidence based medicine: An approach to clinical problem-solving. BMJ: British Medical Journal. 1995;310(6987):1122

[3] Leape LL, Berwick DM, Bates DW. What practices will most improve safety?: Evidence-based medicine meets patient safety. JAMA. 2002;288(4):501-507

[4] Accreditation Council for Graduate Medical Education. ACGME program requirements for graduate medical education in pediatrics. 2013:26. Available from: https://www.appd.org/home/PDF/ ACGMEPediatricRequirements7_2011. pdf

[5] Green ML. Evidence-based medicine training in internal medicine residency programs. Journal of General Internal Medicine. 2000;15(2):129-133

[6] Amgad M et al. Medical student research: An integrated mixed-methods systematic review and meta-analysis. PLoS One. 2015;10(6):e0127470

[7] Applegate WB, Williams ME. Career development in academic medicine. The American Journal of Medicine. 1990;88(3):263-267

[8] Kanna B et al. The research rotation: Competency-based structured and novel approach to research training of internal medicine residents. BMC Medical Education. 2006;6(1):52

[9] Monn MF et al. ACGME core competency training, mentorship, and research in surgical subspecialty fellowship programs. Journal of Surgical Education. 2013;70(2):180-188
[10] Rothberg MB. Overcoming the obstacles to research during residency: What does it take? JAMA. 2012;308(21):2191-2192

[11] Blumenthal DM et al. Addressing the leadership gap in medicine: Residents' need for systematic leadership development training. Academic Medicine. 2012;87(4):513-522

[12] Batalden P et al. General competencies and accreditation in graduate medical education. Health Affairs. 2002;21(5):103-111

[13] Grady EC et al. Defining scholarly activity in graduate medical education. Journal of Graduate Medical Education. 2012;4(4):558-561

[14] Amrhein $\mathrm{T}$ et al. Instituting a radiology residency scholarly activity program. Education for Health. 2015;28(1):68-73

[15] Ledford CJW et al. Resident scholarship expectations and experiences: Sources of uncertainty as barriers to success. Journal of Graduate Medical Education. 2013;5(4):564-569

[16] Stevenson MD et al. Increasing scholarly activity productivity during residency: A systematic review. Academic Medicine. 2017;92(2):250-266

[17] Robbins MS et al. A dedicated scholarly research program in an adult and pediatric neurology residency program. Neurology. 2017;88(14):1366

[18] Manring MM, Panzo JA, Mayerson JL. A framework for improving resident research participation and scholarly output. Journal of Surgical Education. 2014;71(1):8-13

[19] Ross RG, Greco-Sanders L, Laudenslager M. An institutional postdoctoral research training 
program: Increasing productivity of postdoctoral trainees. Academic Psychiatry: The Journal of the American Association of Directors of Psychiatric Residency Training and the Association for Academic Psychiatry. 2016;40(2):207-212

[20] Penrose LL et al. An incremental approach to improving scholarly activity. Journal of Graduate Medical Education. 2012;4(4):496-499

[21] Leshner AI. Standards for Postdoc Training. American Association for the Advancement of Science. 2012:336(6079):276-276

[22] JHU. Core Competencies for Postdoctoral Research Fellows. 2018. Available from: https://www. hopkinsmedicine.org/som/offices/pda/ index.htm

[23] Frey K et al. The 'Collaborative Care' curriculum: An educational model addressing key ACGME core competencies in primary care residency training. Medical Education. 2003;37(9):786-789

[24] Takahashi O et al. Residents' experience of scholarly activities is associated with higher satisfaction with residency training. Journal of General Internal Medicine. 2009;24(6):716-720

[25] Temte J, Hunter P, Beasley J. Factors associated with research interest and activity during family practice residency. Family Medicine. 1994;26(2):93-97

[26] Heitkamp DE et al. A leadership development program for radiology residents. Journal of the American College of Radiology. 2017;14(11):1468-1470

[27] Bruckmann C, Sebestyén E. Ten simple rules to initiate and run a postdoctoral association. PLoS Computational Biology. 2017;13(8):e1005664
[28] Stewart PM. Academic medicine: A faltering engine: Action is needed to respond to growing need and opportunities. BMJ: British Medical Journal. 2002;324(7335):437

[29] Residency Review Committee. ACGME Program Requirements for Graduate Medical Education in Internal Medicine. 2012. Available from: https:// www.acgme.org/Portals/0/PFAssets/ ProgramRequirements/140_internal_ medicine_2017-07-01.pdf

[30] Maizes V et al. The integrative family medicine program: An innovation in residency education. Academic Medicine. 2006;81(6):583-589

[31] Elman NS, Illfelder-Kaye J, Robiner WN. Professional development: Training for professionalism as a foundation for competent practice in psychology. Professional Psychology: Research and Practice. 2005;36(4):367

[32] Verderame MF et al. Point of view: Competency-based assessment for the training of $\mathrm{PhD}$ students and earlycareer scientists. eLife. 2018;7:e34801

[33] Simasek $M$ et al. Meeting resident scholarly activity requirements through a longitudinal quality improvement curriculum. Journal of Graduate Medical Education. 2015;7(1):86-90

[34] Carek PJ et al. Addressing the scholarly activity requirements for residents: One program's solution. Journal of Graduate Medical Education. 2011;3(3):379-382

[35] Emerick T et al. Scholarly activity points: A new tool to evaluate resident scholarly productivity. British Journal of Anaesthesia. 2013;111(3):468-476

[36] Philibert I et al. Scholarly activity in the next accreditation system: Moving from structure and process to outcomes. Journal of Graduate Medical Education. 2013;5(4):714-717 
The Importance of Post-Doctoral Program to GME in an Academic Medical Center DOI: http://dx.doi.org/10.5772/intechopen. 84138

[37] Toledo P et al. Evaluation of the foundation for anesthesia education and research medical student anesthesia research fellowship program participants' scholarly activity and career choices. Anesthesiology: The Journal of the American Society of Anesthesiologists. 2016;124(5):1168-1173

[38] Tachibana C. new tools for measuring academic performance. Science. 2017;355(6325):651-654

[39] Ranieri V, Barratt H, Fulop N, Rees G. Clinical academics' postdoctoral career development. BMJ: British Medical Journal. 2015;351(h6945) 


\section{Edited by Stanislaw P. Stawicki, Michael S. Firstenberg, James P. Orlando and Thomas J. Papadimos}

Graduate medical education (GME) is a continually evolving, highly dynamic area within the complex fabric of the modern health-care environment. Given the rapidly changing regulatory, financial, scientific and technical aspects of GME, many institutions and programs face daily challenges of "keeping up" with the most recent developments within this ever-more-sophisticated operational environment. Organizational excellence is a requirement for the seamless functioning of GME programs, especially when one consider the multiple disciplines and stakeholders involved.

The goal of the current book cycle, titled Contemporary Topics in Graduate Medical Education, beginning with this inaugural tome, is to provide GME professionals with a practical and readily applicable set of reference materials. More than 20 distinguished authors from some of the top teaching institutions in the US, touch upon some of the most relevant, contemporary, and at times controversial topics, including provider burnout, gender equality issues, trainee wellness, scholarly activities and requirements, and many other theoretical and practical considerations.

We hope that the reader will find this book to be a valuable and high quality resource of a broad range of GME-related topics. It is the Editors' goal to create a multi-tome platform that will become the definitive go-to reference for professionals navigating the complex landscape of modern graduate medical education. 